\title{
Incoherent Broad-Band Cavity-Enhanced Absorption Spectroscopy
}

\author{
von \\ Dipl. - Phys. Sven E. Fiedler \\ aus Berlin \\ Der Fakultät II - Mathematik und Naturwissenschaften \\ der Technischen Universität Berlin \\ zur Verleihung des akademischen Grades \\ Doktor der Naturwissenschaften \\ - Dr. rer. nat. - \\ genehmigte Dissertation
}

Berlin 2005

D83 


\title{
Incoherent Broad-Band Cavity-Enhanced Absorption Spectroscopy
}

\author{
von \\ Dipl. - Phys. Sven E. Fiedler \\ aus Berlin \\ Der Fakultät II - Mathematik und Naturwissenschaften \\ der Technischen Universität Berlin \\ zur Verleihung des akademischen Grades \\ Doktor der Naturwissenschaften \\ - Dr. rer. nat. - \\ genehmigte Dissertation
}

Promotionsausschuß

Vorsitzender: Prof. Dr. Erwin Sedlmayr

Berichter: $\quad$ Prof. Dr. Achim Hese

Berichter: $\quad$ Prof. Dr. Peter Zimmermann

Arbeit eingereicht am:

22.12 .2004

Tag der wissenschaftlichen Aussprache: 07.02.2005

Berlin 2005

D83 
Parts of this thesis were already published or presented at conferences:

1. E.W. Gash, M.W.D. Mansfield, A.A. Ruth, M. Staak, S.E. Fiedler, A. Hese and T.G. Shin; "Unusual time-dependent absorption of naphthalene-gas mixtures after UV Flash Photolysis", Poster, Norddeutscher Lasertag, Ham$\operatorname{burg}(2001)$

2. A.A. Ruth, E.W. Gash, M. Staak and S.E. Fiedler

"Multi-photon UV photolysis of naphthalene-gas mixtures: A new gas-phase oscillatory system", Phys. Chem. Chem. Phys., 4 (2002) 5217-5220

3. S.E. Fiedler, A. Hese and A.A. Ruth

"Incoherent broad-band cavity-enhanced absorption spectroscopy", Chem.

Phys. Lett., 371 (2003) 284-294

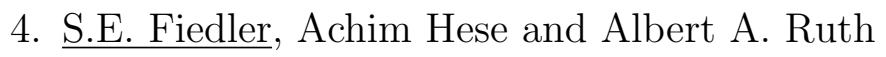

"Incoherent Broadband Cavity Enhanced Absorption Spectroscopy (IBBCEAS)",

Talk (MO 1.4),

"Hochempfindliche Absorptionsspektroskopie an Azulen und O2 mit Hilfe der IBBCEAS” Poster (MO 12.13), DPG-Frühjahrstagung, Hannover (2003)

5. $\underline{\text { S.E. Fiedler }}$

"Incoherent broad-band cavity-enhanced absorption spectroscopy", invited by Dr. H. Linnartz, talk at the Laser Centre VU Amsterdam, Holland (2003)

6. S.E. Fiedler, G. Hoheisel, A.A. Ruth, A. Hese

"Incoherent broad-band cavity-enhanced absorption spectroscopy", Poster, D2 Conf. Proceed. 18th Colloquium On High Resolution Molecular Spectroscopy, Dijon, France, September (2003). 
7. S.E. Fiedler, G. Hoheisel, A.A. Ruth, A. Hese

"Incoherent broad-band cavity-enhanced absorption spectroscopy of azulene in a supersonic jet", Chem. Phys. Lett., 382 (2003) 447-453

8. $\underline{\text { S.E. Fiedler }}$

"IBBCEAS an Flüssigkeiten", talk (MO 3.7), DPG-Frühjahrstagung, München, 2004

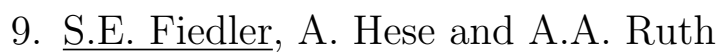

"Incoherent broad-band cavity-enhanced absorption spectroscopy of liquids", submitted to Rev. Sci. Instrum., (2004)

10. S.E. Fiedler

"IBBCEAS", invited talk, Cavity-Ring-Down User Meeting 2004, Heeze, Netherlands

\section{Patent}

"Resonatorverstärktes Absorptions-Spektrometer", German Patent DE 10255 022.0 and International Patent PCT/EP 03/13174, both pending, priority date: 25th Nov. 2002 


\section{Zusammenfassung}

In dieser Arbeit wird eine neue hochempfindliche Spektroskopie-Methode vorgestellt, die sogenannte "Incoherent Broad-Band Cavity-Enhanced Absorption Spectroscopy" (Inkohärente und breitbandige resonatorverstärkte Absorptionsspektroskopie, kurz: IBBCEAS). Diese Technik vereint die Einfachheit und Zuverlässigkeit der klassischen Absorptionsspektroskopie mit der Empfindlichkeit der "Cavity-Ring-Down" Spektroskopie. Das Meßprinzip wurde theoretisch betrachtet und die hergeleiteten Gleichungen experimentell an flüssigen und gasförmigen Proben, sowie bei Messungen an einem Überschall-Düsenstrahl überprüft.

Anhand des sehr schwachen $\mathrm{b}^{1} \Sigma_{g}^{+}\left(v^{\prime}=2\right) \leftarrow \mathrm{X}^{3} \Sigma_{g}^{-}\left(v^{\prime \prime}=0\right)$ triplet-singlet Übergangs von molekularem Sauerstoff konnte bei Spiegelreflektivitäten von $R=0.9999$ für die Messung des Absorptionskoeffizienten eine Empfindlichkeit von $3 \times 10^{-8} \mathrm{~cm}^{-1}$ bei einer spektralen Auflösung von $0.66 \mathrm{~cm}^{-1}(\approx 0.026 \mathrm{~nm})$ demonstriert werden. Vor kurzem wurde ein komplexes oszillierendes Absorptionsverhalten von gasförmigem Naphthalin in einem Edelgas entdeckt, verursacht durch eine MehrPhotonen-Photolyse. Mithilfe von IBBCEAS wurden erste zeitaufgelöste Spektren aufgenommen. Anhand der Spekren konnten einige Moleküle, die als Verursacher der oszillierenden Absorption in Betracht kamen, ausgeschlossen werden. Es wurden Anzeichen für eine autokatalytische chemische Reaktion entdeckt, welche die Oszillationen verursachen könnte, an der mindestens zwei unterschiedliche Moleküle beteiligt sind.

Die Anwendung von IBBCEAS für Absorptionsmessungen an kalten isolierten Molekülen in einem kontinuierlichen Überschall-Düsenstrahl wurde anhand des $\mathrm{S}_{1} \leftarrow \mathrm{S}_{0}$ Übergangs von Azulen demonstriert. Das Absorptionsspektrum wurde über den breiten spektralen Bereich zwischen 17300 und $20255 \mathrm{~cm}^{-1}$ mit einer Auflösung von $\approx 4 \mathrm{~cm}^{-1}$ gemessen. Die beobachteten Absorptionsstrukturen stimmen erstaunlich gut mit einem theoretischen Spektrum überein, das auf der Grundlage von identifizierten vibronischen Übergängen berechnet wurde. 


\section{Summary}

In this work a new highly sensitive method called "Incoherent Broad-Band CavityEnhanced Absorption Spectroscopy" (IBBCEAS) is presented. The technique combines the simplicity and robustness of standard non-invasive optical absorption spectroscopy with the enhancement concepts developed in cavity ring-down spectroscopy. The basic equations to handle the IBBCEA technique were derived and in proof-of-principle experiments verified for liquid and gaseous samples as well as for measurements in a supersonic jet.

By measuring the very weak $\mathrm{b}^{1} \Sigma_{g}^{+}\left(v^{\prime}=2\right) \leftarrow \mathrm{X}^{3} \Sigma_{g}^{-}\left(v^{\prime \prime}=0\right)$ triplet-singlet transition of molecular oxygen with mirrors of $R=0.9999$ and a spectrometer resolution of $0.66 \mathrm{~cm}^{-1}(\approx 0.026 \mathrm{~nm})$, a sensitivity of $3 \times 10^{-8} \mathrm{~cm}^{-1}$ for the absorption coefficient was demonstrated.

Using IBBCEAS, first spectra were recorded as a function of time of the recently discovered complex oscillatory absorption behaviour induced by multi-UV-photon photolysis in a closed gas-phase system of naphthalene in a nobel gas. Some molecules suspected to be the carrier of the absorption could be ruled out and signs for an autocatalytic chemical reaction scheme of at least two species causing the oscillations were found.

The application of IBBCEAS to isolated jet-cooled gas phase species was demonstrated on basis of the $\mathrm{S}_{1} \leftarrow \mathrm{S}_{0}$ transition of azulene in a continuous supersonic expansion. The absorption was measured over a broad spectral range between 17300 and $20255 \mathrm{~cm}^{-1}$ with a resolution of $\approx 4 \mathrm{~cm}^{-1}$. Most of the observed absorption features could be reproduced by a calculated spectrum, basing on identified fundamental vibronic transitions. 


\section{Contents}

$\begin{array}{lll}1 & \text { Introduction } & \mathbf{1 0}\end{array}$

1.1 Conventional Absorption Methods . . . . . . . . . . . . . . . . 11

1.2 Cavity-Enhanced Methods . . . . . . . . . . . . . . . . . . . . . . 12

1.3 Outline of this work . . . . . . . . . . . . . . . . . . . . . . . . . . 13

$\begin{array}{lll}2 & \text { Theory } & 15\end{array}$

2.1 Absorption Spectroscopy . . . . . . . . . . . . . . . . . . . . . . 15

2.2 Principle of CRDS $\ldots \ldots \ldots \ldots$. . . . . . . . . . . . . . . 17

$2.2 .1 \quad$ Sensitivity and Detection Limits . . . . . . . . . . . . . . 21

$2.2 .2 \quad$ Applications and Developments of CRDS . . . . . . . . . . . 22

2.3 Principle of IBBCEAS $\ldots \ldots \ldots \ldots$. . . . . . . . . . . . . . . . . . . . . 24

2.3 .1 The Transmitted Light . . . . . . . . . . . . . . . . . . . 24

2.3 .2 The Absorption Coefficient . . . . . . . . . . . . . . . . . . 26

2.3 .3 The Enhancement of a Spectrometer by IBBCEAS . . . . . 28

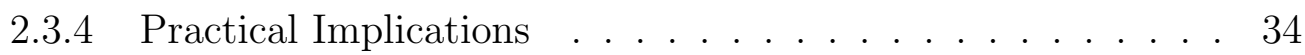

3 Oxygen and Azulene in the Gas Phase 36

$3.1 \quad$ Experimental Setup . . . . . . . . . . . . . . . . . . . 36

3.2 IBBCEAS of Molecular Oxygen . . . . . . . . . . . . . . . . . . . 39

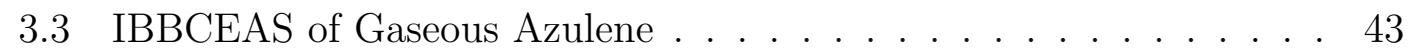

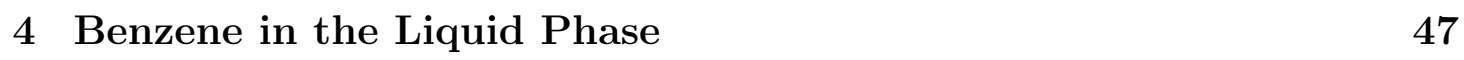

4.1 Cuvette Windows in a Cavity . . . . . . . . . . . . . . . . . . . . . 49

4.2 IBBCEA Setup in a Spectrometer . . . . . . . . . . . . . . . . . 51

4.3 Results . . . . . . . . . . . . . . . . . . . . . . . . . . . . . . . . 54

4.4 Discussion $\ldots \ldots \ldots \ldots \ldots$

4.5 Summary and Outlook $\ldots \ldots \ldots \ldots$ 
5 Naphthalene Gas Phase Oscillations $\quad 60$

$5.1 \quad$ CRDS - Setup . . . . . . . . . . . . . . . . . . 62

5.2 CRDS - Results and Classification of Oscillation Types . . . . . . . 64

5.3 CRDS - Discussion . . . . . . . . . . . . . . . . . . . 67

5.4 IBBCEAS - Setup . . . . . . . . . . . . . . . . . . . 69

5.5 IBBCEAS - The Absorption Spectrum as a Function of Time. . . . 70

5.6 IBBCEAS - Fit of the Spectrum . . . . . . . . . . . . . . . 71

5.7 IBBCEAS - Time Dependence of the Absorption Features . . . . . 75

5.8 IBBCEAS - Comparison to known spectra . . . . . . . . . . . . 78

5.9 Summary $\ldots \ldots \ldots \ldots$. . . . . . . . . . . . . . . . . . . . 82

5.10 Future Work . . . . . . . . . . . . . . . . . . . . . . . . . . . 83

$\begin{array}{llr}6 & \text { Azulene in a Supersonic Jet } & 85\end{array}$

6.1 Motivation . . . . . . . . . . . . . . . . . . . . . . . . 88

6.2 The Conical Intersection . . . . . . . . . . . . . . . . . . . . . . . . 89

6.3 Experimental Setup . . . . . . . . . . . . . . . . . . . . . . . . . . . 91

6.4 IBBCEAS in Comparison to CRDS Measurements . . . . . . . . . . 94

6.5 Variation of Experimental Parameters . . . . . . . . . . . . . . . . 96

6.6 Results of IBBCEAS of Azulene in a Supersonic Jet . . . . . . . . . 100

6.7 Calculation of the Azulene Spectrum . . . . . . . . . . . . . . . 103

6.8 Discussion . . . . . . . . . . . . . . . . . . . . . . 106

6.9 Summary $\ldots \ldots \ldots \ldots$. . . . . . . . . . . . . . . . . . . 110

$\begin{array}{lll}7 & \text { Prospects of the IBBCEA technique } & 111\end{array}$

$\begin{array}{lll}8 & \text { Conclusion } & 115\end{array}$

\begin{tabular}{lr}
\hline A Appendix & 118
\end{tabular}

A.1 Mirror Reflectivities . . . . . . . . . . . . . . . . . . . . . . . . . . 118

A.2 Nonlinear Chemical System . . . . . . . . . . . . . . . . . 120

A.3 Azulene: Vibronic Absorption Lines . . . . . . . . . . . . . . . . . . 122

\begin{tabular}{lr}
\hline Bibliography & 126
\end{tabular}

\begin{tabular}{lr}
\hline Curriculum Vitae & 138
\end{tabular}

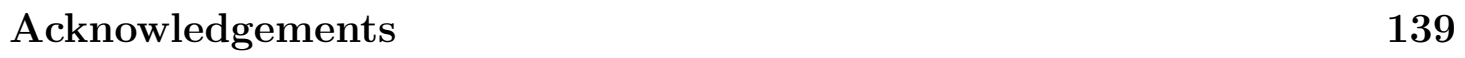




\section{Acronyms and Remarks}

\begin{tabular}{|c|c|}
\hline ASE & - Amplified Spontaneous Emission \\
\hline CEAS & - Cavity Enhanced Absorption Spectroscopy \\
\hline CRDS & - Cavity Ring-Down Spectroscopy \\
\hline $\mathrm{cw}$ & - continuous-wave \\
\hline DIB & - Diffuse Interstellar Band \\
\hline FSR & - Free Spectral Range \\
\hline FWHM & - Full Width at Half Maximum \\
\hline IBBCEAS & $\begin{array}{l}\text { - Incoherent Broad-Band Cavity-Enhanced Absorption } \\
\text { Spectroscopy }\end{array}$ \\
\hline ICOS & - Integrated Cavity Output Spectroscopy \\
\hline IR & - Infrared \\
\hline LIF & - $\quad$ Laser Induced Fluorescence \\
\hline $\mathrm{PAH}$ & Polycyclic Aromatic Hydrocarbon \\
\hline REMPI & Resonance Enhanced Multi-Photon Ionisation \\
\hline SNR & - Signal-to-Noise Ratio \\
\hline UV & - Ultraviolet \\
\hline VIS & - Visible \\
\hline wavenumber & $\begin{array}{l}\text { As usual for molecular spectroscopic publications, } \\
\text { wavenumbers are used here to represent the energy re- } \\
\text { lated to spectral lines: } E=h \cdot c \cdot \widetilde{\nu} \text {. The wavenumber } \\
\left([\widetilde{\nu}]=\mathrm{cm}^{-1}\right) \text { is the reciprocal of the wavelength } \lambda \text {, while } \\
h \text { is the Planck constant and } c \text { the speed of light. }\end{array}$ \\
\hline
\end{tabular}




\section{Chapter 1}

\section{Introduction}

For more than a century spectroscopy has been recognized as a fundamental field of science for the investigation of atomic and molecular properties. Most of our present knowledge on the molecular structure and dynamics of matter was derived from studying the interaction of electromagnetic radiation with matter, by looking at the processes of absorption, emission or scattering.

Wavelength measurements of spectral absorption and emission lines allow the determination of energy levels of atomic or molecular systems. The absorption strength is proportional to the transition probability, which measures how strongly the two levels of a molecular transition are connected by the transition moment. Since the transition probability depends on the wave functions of both levels, intensity measurements are useful to verify the spatial charge distribution of excited electrons, which can only be roughly calculated from approximate solutions of the Schrödinger equation. Similar approaches allow also to investigate the geometry of molecules using transitions between vibrational and rotational energy levels.

Today, direct optical absorption spectroscopy is widely used in chemical, biological, atmospheric and geophysical sciences. It is a straightforward non-invasive 
approach for the in-situ detection of trace species concentrations and the measurement of wavelength dependent cross-sections. Other common spectroscopical techniques, basing on the detection of phenomena induced by the absorption of light, e.g. fluorescence, do not give the absolute but the relative absorptions only. Generally two conceptually different experimental ways of measuring the absolute absorption of a gaseous sample can be distinguished:

\subsection{Conventional Absorption Methods}

In "conventional" optical absorption experiments the incident and transmitted intensity of a beam of light, travelling through a medium of a certain path length, are measured. The absorption is related to the attenuation of the light expressed in terms of Lambert-Beer's law, assuming light scattering to be a negligible attenuation mechanism. In this approach the measurement of the absorption is genuinely intensity dependent and its sensitivity depends linearly on the optical path length, which is therefore sought to be maximized in virtually all practical applications. Long path lengths are thus the key to the most sensitive established atmospheric pollution and trace gas monitoring methods. Classical experimental schemes employ multi-pass cells according to White [White42, White76] or Herriot and Schulte [Herriot65]. Many modifications and improvements of these original setups have been developed [Chernin01, Steyert01, which are associated with a loss of experimental simplicity. Many long path length approaches use narrow-band tunable diode-lasers (TDLAS [Reid78, Werle98]) in combination with multi-pass cells or in open path experiments. Multi-pass absorption spectroscopy is also combined with amplitude or frequency modulation spectroscopy (AMS and FMS respectively) Bjorklund80, Hall81, which achieves sufficient sensitivity for detection of certain atmospheric trace gases. Spectrally broad (white) light sources, whose 
emission is dispersed before or after passing the sample, are also used, for instance in differential optical absorption spectroscopy (DOAS) [Platt94].

\subsection{Cavity-Enhanced Methods}

"Classical" cavity ring-down spectroscopy (CRDS) O'Keefe88, which is insensitive to intensity fluctuations, underwent a rapid development over the past decade. Various types of cavity-enhanced methods, based on pulsed and cw laser excitation [Romanini97a] of the sample gas were established, summaries can be found in several reviews [Scherer97, Wheeler98, Busch99, Berden00, Ball03]. Among the continuous wave cavity-enhanced techniques significant improvements were made in recent years using diode lasers [Ye98, whereby exceptional sensitivities were achieved in applications such as NICE-OHMS [Ma99] or heterodyne CRDS [Ye00]. However, refinement of the spectral resolution (better than $1 \mathrm{MHz}$ ) and enhancements in terms of sensitivity are commonly achieved at the expense of experimental simplicity $\overleftarrow{C}$. In diode laser based cavity-enhanced absorption spectroscopy (CEAS), as published by Engeln et al. Engeln98d the cavities' transmission is detected and extensively averaged, similar to the pulsed integrated cavity output analysis (ICOS) demonstrated by O'Keefe O'Keefe98. Thereby a level of experimental simplicity as well as a high level of sensitivity is retained. However, although CEAS is promising in terms of robustness and spectral resolution, it cannot be flexibly applied in a wide spectral region (particularly not

\footnotetext{
a Noise-Immune Cavity-Enhanced Optical Heterodyne Modulation Spectrometry.

b The signal is modulated with two or more different frequencies and detected phase-sensitive.

c In many applications the mode-locking of the cavity is necessary, which requires feedback electronics, fast optical switches, etc., also Faraday rotators are commonly used to avoid feedback into the laser diode.

d The same approach was somewhat later published again and referred to as integrated cavity output spectroscopy, cw-ICOS ['Keefe99.
} 
at short wavelengths); also mode-hops in the laser's semi-conductor material represent a practical drawback, especially if a high resolution is not required and a large spectral region is to be studied. In atmospheric applications where several compounds are to be monitored simultaneously or species with broad absorption bands are to be detected, techniques covering wide spectral absorption regions are required, rather than high spectral resolution. Therefore, and due to multiplexing possibilities, the development of broad-band cavity-enhanced methods appear very attractive and several pulsed laser applications have been developed [Engeln96, O'Keefe98, Crosson99, Scherer98, Czyzewski01], besides more established intracavity absorption methods [Romanini95, Cheskis99]. With two very recent exceptions on fourier transform phase shift CRDS [Hamers02] and broadband CEAS using light emitting diodes Ball04 all previous cavity-enhanced absorption techniques were laser based.

\subsection{Outline of this work}

In this work a new highly sensitive method called Incoherent Broad-Band CavityEnhanced Absorption Spectroscopy (IBBCEAS) is presented. IBBCEAS is able to use a simple white incoherent light source. A cavity, in which a sample can be placed, enhances the effective absorption path length up to a factor of 10000 . On the other hand the light intensity is strongly reduced. The light coming out of the cavity is dispersed by a conventional spectrometer and detected over a broad wavelength region at the same time (broad-band) with a photodiode array.

Section 2.3 presents the measurement principle of IBBCEAS. The theoretical limits of the enhancement of the signal-to-noise ratio of IBBCEAS in comparison with single pass absorption experiments are discussed for a set of given experimental cavity parameters. The features of the new technique are demonstrated on basis 
of the absorption spectra of benzene in the liquid phase (chapter 4) and gas phase spectra of azulene and oxygen (section 3.3 and 3.2 .

New scientific results are reported in chapter 5 concerning the recently discovered complex oscillatory absorption behaviour induced by UV multi-photon photolysis in a closed gas-phase system of naphthalene in a noble gas. The phenomenon was found at preliminary experiments by accident and was first investigated by CRDS [Ruth02b, Gash04] at one wavelength only. In this work first IBBCEA spectra of the oscillating absorption are presented as a function of time.

The spectrum of azulene in a pulsed supersonic jet was measured in the research group of Hese between $14283 \mathrm{~cm}^{-1}$ and $17300 \mathrm{~cm}^{-1}$ using CRDS [Ruth99. In chapter 6IBBCEA spectra of azulene in a continuous supersonic jet are shown at high excess energies and compared to the CRD results. The IBBCEA measurements cover the interesting spectral region at high excess energies $\left(>3000 \mathrm{~cm}^{-1}\right.$ above the $\mathrm{S}_{1}$ origin). In that region a conical intersection of the $\mathrm{S}_{0}$ and $\mathrm{S}_{1}$ potentials leads to very short lifetimes and therefore to homogeneously broadened line widths.

In chapter 7 some possible future modifications and applications of IBBCEAS are discussed and chapter 8 concludes this work. 


\section{Chapter 2}

\section{Theory}

This chapter contains basic considerations about absorption and its associated physical values. For a better comprehension of the technique used in this work, the methods of conventional single pass spectroscopy and cavity ring-down absorption spectroscopy (CRDS) as an example for cavity-enhanced absorption techniques are briefly described. Subsequently the principle of the new Incoherent Broad-Band Cavity-Enhanced Absorption Spectroscopy (IBBCEAS) is discussed and mathematical formulas are developed for the physical description of the system.

\subsection{Absorption Spectroscopy}

Molecules and atoms absorb electromagnetic radiation at specific frequencies. Each compound has its own unique spectrum of frequency dependent absorption transitions associated with its electronic, vibrational and rotational properties (refer e.g. [Hollas96]). In a conventional direct optical absorption experiment the incident and transmitted intensities ( $I_{0}$ and $I$, respectively) of a light beam, propagating through a medium of path length $d$, are measured. The absorption of the medium 
is related to the attenuation of the light intensity which is expressed by the well known Lambert-Beer law:

$$
I(\lambda)=I_{\text {in }}(\lambda) \cdot \exp [-\alpha(\lambda) \cdot d]
$$

where $\alpha(\lambda)$ is the wavelength dependent absorption coefficient, which is usually given in $\mathrm{cm}^{-1}$. The absorption coefficient $\alpha(\lambda)=n \sigma(\lambda)$ is normally expressed as the product of the number density $n$ and the specific absorption cross-section $\sigma(\lambda)$ of the absorbing species ${ }^{\mathfrak{a}}$. The absorption coefficient is also commonly expressed in molar quantities $\alpha(\lambda)=C \cdot \epsilon(\lambda)$, where $C$ is the concentration and $\epsilon(\lambda)$ the molar extinction coefficient of the absorbing species. The unitless product $A(\lambda)=\alpha(\lambda) \cdot d$, called "absorbance" or "optical density", is the normalized loss of light intensity per pass through the medium.

From Eq. 2.1 the following equation for the absorption coefficient can be obtained:

$$
\begin{aligned}
\alpha(\lambda) & =-\frac{1}{d} \ln \left(\frac{I(\lambda)}{I_{\mathrm{in}}(\lambda)}\right) \\
& \approx \frac{1}{d}\left(1-\frac{I(\lambda)}{I_{\mathrm{in}}(\lambda)}\right)
\end{aligned}
$$

The smallest detectable absorption is limited by the minimum fractional change in intensity that can be measured. For inherently weak absorptions $\left(1-\frac{I}{I_{\mathrm{in}}}<10^{-6}\right)$ it is difficult to achieve an adequate signal-to-noise ratio to distinguish the difference between $I_{\text {in }}$ and $I$ and the sensitivity is generally limited by fluctuations in $I_{\text {in }}$. It is clear that a long path length would result in a larger intensity difference and thus an increased sensitivity.

$\overline{\text { a }} \quad[\alpha(\lambda)]=\mathrm{cm}^{-1},[n]=\mathrm{cm}^{-3},[\sigma(\lambda)]=\mathrm{cm}^{2},[C]=\mathrm{mol} / \mathrm{dm}^{3},[\epsilon(\lambda)]=\mathrm{dm}^{3} \mathrm{~mol}^{-1} \mathrm{~cm}^{-1}$ 


\subsection{Principle of Cavity Ring-Down Spectroscopy}

Cavity ring-down spectroscopy (CRDS) is the name for a highly sensitive method for direct absorption measurements [Busch99]. The absorption is determined by measuring the decay-rate of light transmitted through a stable optical resonator, formed by two highly reflecting mirrors, enclosing the gaseous sample under investigation. Since the experimental principle is based on the measurement of a rate rather than a magnitude of an intensity change, this approach virtually eliminates the problem of light intensity fluctuations, which are common in conventional absorption spectroscopy. This, combined with the ability to use very long effective path lengths, gives CRDS its high sensitivity. Recently many developments and refinements to the cavity ring-down technique have expanded its versatility and have allowed it to be used in a much wider range of studies. However, here an introduction is given only to pulsed CRDS. For a detailed overview of the different existing varieties of CRD and other cavity-enhanced schemes see for instance the references [Berden00] and Ball03].

The experimental scheme for pulsed CRDS is shown in Fig. 2.1. A short pulse ( $\sim 10 \mathrm{~ns})$ of a tunable laser is coupled into an optically stable high-finesse cavity formed by two highly reflective dielectric mirrors which are separated by a distance $d$. A small fraction of the laser pulse intensity enters the cavity through the front mirror. This pulse travels back and forth between the two highly reflecting mirrors. If the reflectivity $\mathrm{R}(\lambda)$ of the mirrors at the wavelength $\lambda$ is high enough (typically $R>0.9999$ in the visible) the light pulse can be stored in the cavity for tens of microseconds, which corresponds to several thousand passes of the pulse depending on the mirror separation $d$. The light intensity in the cavity decreases due to the overall losses of the resonator. This decay is monitored by measuring the transmitted intensity of the light pulses by means of an appropriate detector 


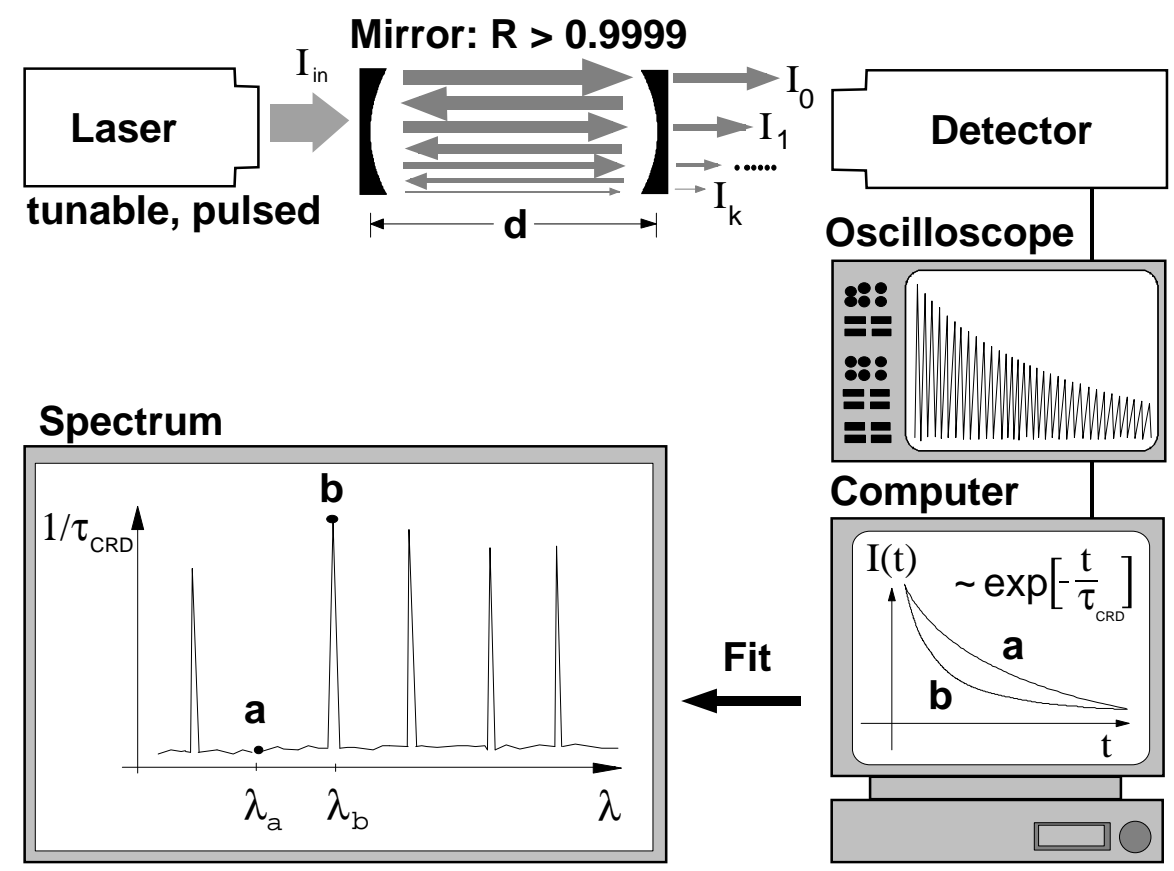

Figure 2.1: Experimental scheme [Ruth00] demonstrating the pulsed cavity ring-down principle. The difference between e.g. the ring-down time at $\lambda_{\mathrm{a}}$ and the ring-down time at $\lambda_{\mathrm{b}}$ is an absolute measure of the absorption at $\lambda_{\mathrm{b}}$ according to Eqs. 2.7 and 2.8, provided other loss mechanisms are negligible.

on the exit side of the resonator. Usually photomultiplier tubes or photodiodes are employed for that purpose. The signal is recorded with a digital oscilloscope and the decay time is analysed by a computer. Two cases have to be distinguished:

(a) empty resonator or no absorption: In an empty (evacuated) resonator or if the sample in the resonator does not absorb at the wavelength $\lambda_{\mathrm{a}}$, the intensity measured after the first pass will be $I_{0}=T^{2} \cdot I_{\text {in }}$, where $T=1-R$ is the transmission of each mirror and $I_{\text {in }}$ is the intensity of the input laser pulse (cf. Fig. 2.1). During each round-trip (= two passes) the pulse intensity is attenuated by a factor of $R(\lambda)^{2}$ leading to an intensity of $I_{k}=I_{0} R^{2 k}=I_{0} \cdot \exp (2 k \cdot \ln [R])$ after $k$ round-trips. Since different mirrors are never of exactly equal reflectivity, $R$ is the geometric average, $\sqrt{R_{1} \cdot R_{2}}$, of the reflectivities of the two mirrors. Replacing the

$\overline{\mathbf{b}} \quad I_{0}=I_{0}(\lambda), I_{\text {in }}=I_{\text {in }}(\lambda), I_{k}=I_{k}(\lambda), T=T(\lambda), R=R(\lambda), R_{1}=R_{1}(\lambda), R_{2}=R_{2}(\lambda)$ 
discrete number of round-trips $k$ by the continuous parameter time, $t=k \cdot(2 d / c)$, leads to an expression for the time-dependence of the laser pulse intensity, which is measured at the resonator exit ( $c$ is the speed of light).

$$
I(t)=I_{0} \cdot \exp \left(\frac{c \ln R}{d} t\right)
$$

The decay time of this mono-exponential decay is called ring-down time

$$
\tau_{\mathrm{CRD}}=\frac{d}{c|\ln R|} \quad, \quad \ln R \approx R-1<0
$$

which is solely dependent on the mirror reflectivity at a given mirror separation $d$. By scanning the laser wavelength and measuring $\tau_{\mathrm{CRD}}(\lambda)$ the wavelengthdependence of the mirror reflectivity can be determined. For the derivation of Eq. 2.4 it was assumed that diffraction losses of the cavity are negligible. This is a valid assumption since in practice almost always resonator conditions can be found where this is the case.

(b) filled resonator: In a resonator which contains a gaseous medium the absorption of the respective species will increase the total losses per pass by $\sim \exp (\alpha \cdot d)$ according to Lambert-Beer's law. It is important that losses due to scattering of the light in the resonator are kept much smaller than the losses due to the limited mirror reflectivity. From the intensity after $k$ round-trips in the cavity, $I_{k}=I_{0} \cdot[R \cdot \exp (\alpha \cdot d)]^{2 k}$, the time-dependence of the intensity leaving the resonator can be written as

$$
I(t)=I_{0} \exp \left(-\frac{\alpha \cdot d+|\ln R|}{d} c \cdot t\right)
$$

$\overline{\mathbf{c}} \quad \tau_{\mathrm{CRD}}=\tau_{\mathrm{CRD}}(\lambda), \alpha=\alpha(\lambda)$ 
In this case the reciprocal ring-down time $\tau_{\text {CRD }}^{-1}$ is proportional to the total losses per round-trip:

$$
\tau_{\mathrm{CRD}}^{-1}=\alpha \cdot c+|\ln R| \cdot \frac{c}{d}
$$

The absorption coefficient of the gaseous medium $\alpha$ is derived by fitting Eqs. 2.4 and 2.6 to the respective measured intensity decay curves for the empty and the filled resonator and subsequently subtracting the respective reciprocal ring-down times (see Fig. 2.1, at $\lambda_{\mathrm{a}}$ is $\alpha\left(\lambda_{\mathrm{a}}\right) \approx 0$ and at $\lambda_{\mathrm{b}}$ is $\alpha\left(\lambda_{\mathrm{b}}\right)>0$ ):

$$
\alpha=\frac{1}{c}\left(\tau_{\mathrm{CRD}}^{-1}(\text { filled })-\tau_{\mathrm{CRD}}^{-1}(\text { empty })\right)
$$

An absorption spectrum is therefore measured by tuning the laser and plotting $\tau_{\text {CRD }}(\lambda)$ versus the wavelength $\lambda$, taking into account the "background" of the empty resonator, $|\ln R(\lambda)| c / d$, which is solely dependent on the mirror reflectivity. CRD absorption spectroscopy is therefore based on the measurement of the rate of absorption rather than the magnitude of absorption of the light pulse confined in the cavity. This is the reason why CRD spectroscopy is practically intensityindependent and only limited by the shot-to-shot noise of the ring-down time. If the absorbing species is not a static gas but for instance a molecular jet or a flame, the mirror separation $d$ must be replaced by the real interaction length of the laser beam with the absorbing species. If the number density $n$ (or concentration $C$ ) of the absorbing species in the interaction region is known, then absolute absorption cross-sections $\sigma(\lambda)$ (or extinction coefficients $\epsilon(\lambda)$ ) can be determined and vice versa. 


\subsubsection{Sensitivity and Detection Limits}

From Eq. 2.6 it becomes obvious that the better the mirror reflectivity, i.e. the longer the ring-down time, the smaller absorption coefficients can be measured. Formally the sensitivity of the CRD method is defined through the relative decrease of the light intensity per pass, $\delta I$, which is related to the quantities in a CRD experiment in the following way (Eq.2.5 in Eq.2.8, refer also to [Zalicki95]):

$$
\begin{aligned}
\delta I & =\frac{I_{0}-I}{I_{0}} \approx \alpha \cdot d \\
& \approx(1-R) \cdot \frac{\tau_{\mathrm{CRD}}(\text { empty })-\tau_{\mathrm{CRD}}(\text { filled })}{\tau_{\mathrm{CRD}}(\text { filled })}
\end{aligned}
$$

The smallest measurable absorption coefficients therefore depend on the smallest detectable change in the ring-down time. In other words, the accuracy with which the ring-down time can be measured determines the size of the smallest measurable absorption coefficient. This is in agreement with the fact, that ring-down times can be determined more accurately the longer the ring-down decays are.

Difficulties in the determination of the ring-down time are (i) alignment of the mirrors, (ii) mode beating as well as saturation effects, (iii) laser light quality (amplified spontaneous emission (ASE) $\mathbf{d}$, superposition of laser modes), (iv) nonlinearities of the detector and (v) electrical noise (e.g. offset corrections). The different contributions of the experimental conditions that increase the error in $\tau_{\mathrm{CRD}}$ are not easy to establish.

The mirrors used for CRDS in this work had a reflectivity of $R=0.9999$ and the cavity was between $45 \mathrm{~cm}$ and $100 \mathrm{~cm}$ long. Typical ring-down times measured

d The spontaneously emitted light is amplified by a single pass through the dye laser medium, but the requirements for a narrow wavelength selection are generally multiple passes through the laser setup. 
were in the order of $20 \mu \mathrm{s}$. This corresponds to an effective path length travelled by the light of $6000 \mathrm{~m}$. The accuracy of the ring-down times achieved in this work is $\sim 1 \%\left(\Delta \tau_{\mathrm{CRD}} / \tau_{\mathrm{CRD}} \approx 0.01\right)$ resulting in a detection limit for $\alpha$ of $\sim 10^{-8} \mathrm{~cm}^{-1}$. Although this is a common value for CRDS, more elaborated setups reported values of $10^{-10} \mathrm{~cm}^{-1}$ in the visible Romanini97b]. The reference [Ye98] even reports an outstanding sensitivity of $10^{-14} \mathrm{~cm}^{-1}$ at $1064 \mu \mathrm{m}$, using a very sophisticated setup. The highest mirror reflectivity, $R=0.9999984$, reported in the literature G. Rempe92 was measured at $852 \mathrm{~nm}$ in a very short cavity $(d=4 \mathrm{~mm})$.

It is important to point out here that CRDS is a highly sensitive method for direct absorption measurements. Other absorption techniques can be more sensitive but are based on the detection of phenomena induced by the absorption of light: e.g. Laser induced fluorescence (LIF) which is able to measure absorptions per pass up to $10^{-15}$ [Demtröder03] and resonant enhanced multi-photon ionisation (REMPI) [Demtröder88]. Both techniques require calibration to obtain quantitative absorption coefficients, CRDS does not.

\subsubsection{Applications and Developments of CRDS}

CRDS with pulsed lasers has demonstrated its versatility and adaptability; it has been used in many different environments and for many varied measurements.

CRDS measurements have been made in open air (for example see ['Keefe88]), cells, molecular jets [Scherer95, Ruth99], plasmas [Miller97] and flames [Meijer94. As well as gas phase species, CRDS has also been used to study the absorption properties of molecular clusters [Paul99, molecules in the liquid Pipino00, Xu02, and solid phase Engeln99 and to investigate the scattering properties of aerosols Sappey98.

Recently the range of application of the pulsed CRD technique has been extended with the introduction of many new variants using for instance a fourier transform 
spectrometer, [Engeln96], polarisation techniques [Engeln97a, a free-electron laser Engeln97b or a monolithic folded resonator for evanescent waves Pipino00. Another development in CRDS should be mentioned here since it is close to the IBBCEA technique presented in this work. The 'cavity enhanced absorption spectroscopy' (CEAS, Engeln98]), also called 'integrated cavity output spectroscopy' (ICOS, O'Keefe98]), allows for absorption measurements to be made without temporal analysis of the ring-down transient. In CEAS the wavelength of a diode laser is scanned continuously. The laser light is coupled into the cavity via accidental coincidences of the light with the cavity eigenmodes. The key concept is that the frequency of the laser should be in resonance with each cavity mode equally long. This can easily be done by scanning the frequency of the laser very fast, so that the interaction time is determined by the scanning speed of the laser and not by the instability of the cavity. Alternatively, the laser can be scanned slowly while the cavity mode structure is scanned by moving one of the cavity mirrors. The time-integrated intensity of the light leaking out of the cavity is measured. Its inverse is linearly proportional to the losses of the cavity. Although coherent light of only one wavelength is used and the integrated output for CEAS is calculated in the time domain by the buildup and decay of the light energy stored in the cavity, a similar equation is derived in this work for a broad-band excitation of the cavity (see section 2.3, Eq. 2.16).

A disadvantage of CEAS is that it is not possible to determine absorption coefficients directly. In order to calculate absorption coefficients either the mirror reflectivities must be known or calibration with molecules of known cross section and number density must be undertaken. 


\subsection{Principle of Incoherent Broad-Band Cavity- Enhanced Absorption Spectroscopy}

An optical cavity is transparent for particular frequencies, even if excited with thermal, totally incoherent light. This fact is based on the superposition principle [Born80 as discussed by Lehmann and Romanini for ring-down cavities [Lehmann96]. The eigenmode structure of an optical cavity exclusively depends on its geometry, and is based on wave interference in the frequency domain (and not in the time domain), which are generally interpreted in terms of cavity resonances. The eigenmode structure is an inherent feature of the cavity and mode formation is independent of the coherence time of the excitation light (see also Hodges96]). The following consideration concerning measurements of absorption coefficients using optical cavities with broad-band incoherent light is based on the superposition principle and therefore valid for all wavelengths. It is assumed that nonlinear effects are negligible and that the bandwidth $\Delta \nu$ of the detector is sufficiently large (i.e. $\Delta \nu>$ free spectral range of the cavity) to be insensitive to intensity variations due to the eigenmode structure of the resonator.

\subsubsection{The Transmitted Light}

We consider an optically stable cavity of length $d$ (see Fig. 2.2), formed by two dielectric mirrors of reflectivity $R_{1}$ and $R_{2}$, which is continuously excited with incoherent light of intensity $I_{i n}$. This light may have any spectral intensity distribution if the transmitted light is spectral analyzed. However, it is sufficient to consider monochromatic light only due to the superposition principle. It is assumed that the dielectric layers of the mirror do not absorb light and, apart from the losses $(1-R)$ due to imperfect mirror reflectivities, the cavity is subject to 


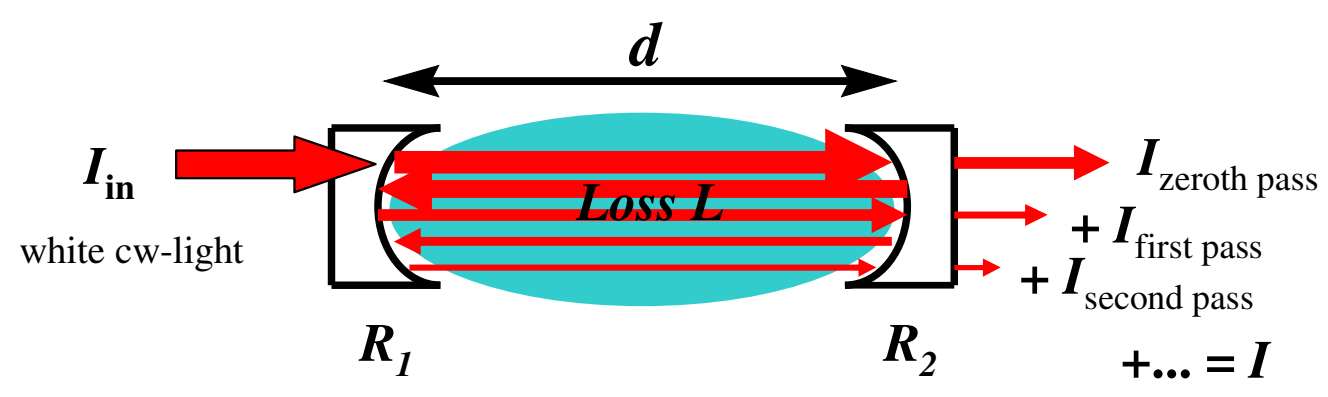

Figure 2.2: Sketch of the IBBCEAS principle.

additional losses $(1-L)$ per pass. The intensity of the light transmitted by the cavity, $I$, can then be described by the sum of the intensities after an even number of reflections in the cavity.

$$
\begin{array}{rlr}
I= & I_{\text {in }}\left(1-R_{1}\right)(1-L)\left(1-R_{2}\right) & \text { (zeroth pass) } \\
& +I_{\text {in }}\left(1-R_{1}\right)(1-L) R_{2}(1-L) R_{1}(1-L)\left(1-R_{2}\right) & \text { (first pass) } \\
& +\ldots \ldots & \\
& + & \\
& +I_{\text {in }}\left(1-R_{1}\right)\left(1-R_{2}\right) R_{2}^{n} R_{1}^{n}(1-L)^{2 n+1} & \\
& +\ldots \ldots & \\
= & I_{i n}\left(1-R_{1}\right)\left(1-R_{2}\right)(1-L) \sum R_{1}^{n} R_{2}^{n}(1-L)^{2 n} &
\end{array}
$$

Since $R_{1}$ and $R_{2}<1$ and $L<1$ this geometric series converges and Eq. (2.11) can be written in the form

$$
I=I_{i n} \frac{\left(1-R_{1}\right)\left(1-R_{2}\right)(1-L)}{1-R_{1} R_{2}(1-L)^{2}}
$$




\subsubsection{The Absorption Coefficient}

For an (empty) resonator with $L=0$, the time-integrated transmitted intensity, $I_{0}$, is given by ${ }^{\mathrm{e}}$

$$
I_{0}=I_{\text {in }} \frac{\left(1-R_{1}\right)\left(1-R_{2}\right)}{1-R_{1} R_{2}} \quad \stackrel{\substack{R_{1} \approx R_{2}=R \\ 1+R \approx 2}}{\approx} \quad I_{\text {in }} \frac{1-R}{2}
$$

and the fractional losses per pass can be expressed as a function of the ratio of intensities measured without and with losses, $I_{0} / I$ :

$$
1-L={ }_{(-)}^{+} \sqrt{\frac{1}{4}\left(\frac{I_{0}}{I} \frac{\left(1-R_{1} R_{2}\right)}{R_{1} R_{2}}\right)^{2}+\frac{1}{R_{1} R_{2}}}-\frac{1}{2} \frac{I_{0}}{I} \frac{\left(1-R_{1} R_{2}\right)}{R_{1} R_{2}}
$$

Assuming the losses per pass to be solely due to (linear) Lambert-Beer absorption, i.e. $(1-L)=\exp (-\alpha d)$, the absorption coefficient, $\alpha$, can be written in the very general form

$$
\alpha=\frac{1}{d}\left|\ln \left(\frac{1}{2 R_{1} R_{2}}\left(\sqrt{4 R_{1} R_{2}+\left(\frac{I_{0}}{I}\left(1-R_{1} R_{2}\right)\right)^{2}}-\frac{I_{0}}{I}\left(1-R_{1} R_{2}\right)\right)\right)\right|
$$

It should be noted that no approximation, neither regarding the size of $\alpha$ nor that of $R$, was made in order to derive Eq. 2.15), which is therefore valid for large absorptions and small reflectivities. The path length $d$ for a single pass through the absorbing species has to be distinguished here from the cavity length $\ell$. Although $d$ and $\ell$ are often the same (e.g. gas cells) they can have very different values (e.g. supersonic jet expansions).

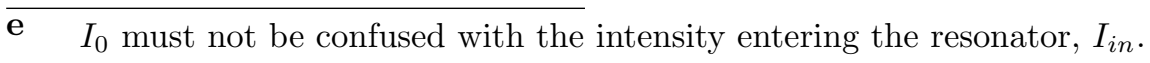


Approximating the ratio $I_{0} / I$ by a Taylor expansion for small losses per pass (see Eq. 2.12 and Eq. 2.13), $L \rightarrow 0$, and high reflectivities of the mirrors, $R \rightarrow 1$, the absorption coefficient in Eq. 2.15 can be approximated by ff

$$
\alpha=\frac{1}{d}\left(\frac{I_{0}}{I}-1\right)\left(1-\sqrt{R_{1} R_{2}}\right)
$$

The deviation of the solid lines calculated by Eq. 2.16 from the dotted line according to Eq. 2.15 due to the approximation for high losses $L$ and small mirror reflectivities $R$ can be seen in figure 2.3. Only for high reflectivities $(R \geq 99.9 \%)$ or small losses $\left(I_{0} / I \leq 2 \hat{\approx} L \leq(1-R)\right)$ the approximation is valid and the lines merge.

The sensitivity of the new IBBCEAS technique can be easily compared to that of conventional single pass absorption spectroscopy as demonstrated by the following equationg,

$$
\alpha_{\min }=\frac{1}{d}\left(1-\frac{I_{\text {min, single }}}{I_{0, \text { single }}}\right)=\frac{1}{d}\left(1-\frac{I_{\text {min,IBBCEAS }}}{I_{0, \text { IBBCEAS }}}\right)\left(1-\sqrt{R_{1} R_{2}}\right)
$$

$I_{\text {min,single }}$ and $I_{\text {min,IBBCEAS }}$ are the minimum detectable intensities smaller than $I_{0, \text { single }}$ and $I_{0}$, respectively. The right side of Eq. 2.17 represents the minimum detectable fractional absorption in IBBCEAS, which follows directly from Eq.2.16. It is equal to the minimum detectable absorption coefficient, $\alpha_{\min }$, which is expressed for a single pass absorption experiment on the left side of Eq. 2.17. If similar signalto-noise ratios (discussed below in detail) of the intensities for both measurements

f A similar formula was given for cavity-enhanced absorption spectroscopy (CEAS), confer Eq. (9) in Ref. Berden00.

$\mathrm{g}$ In the case of very small (minimal) losses the approximation $\left(I_{0}-I\right) / I_{0} \approx\left(I_{0}-I\right) / I$ holds. 


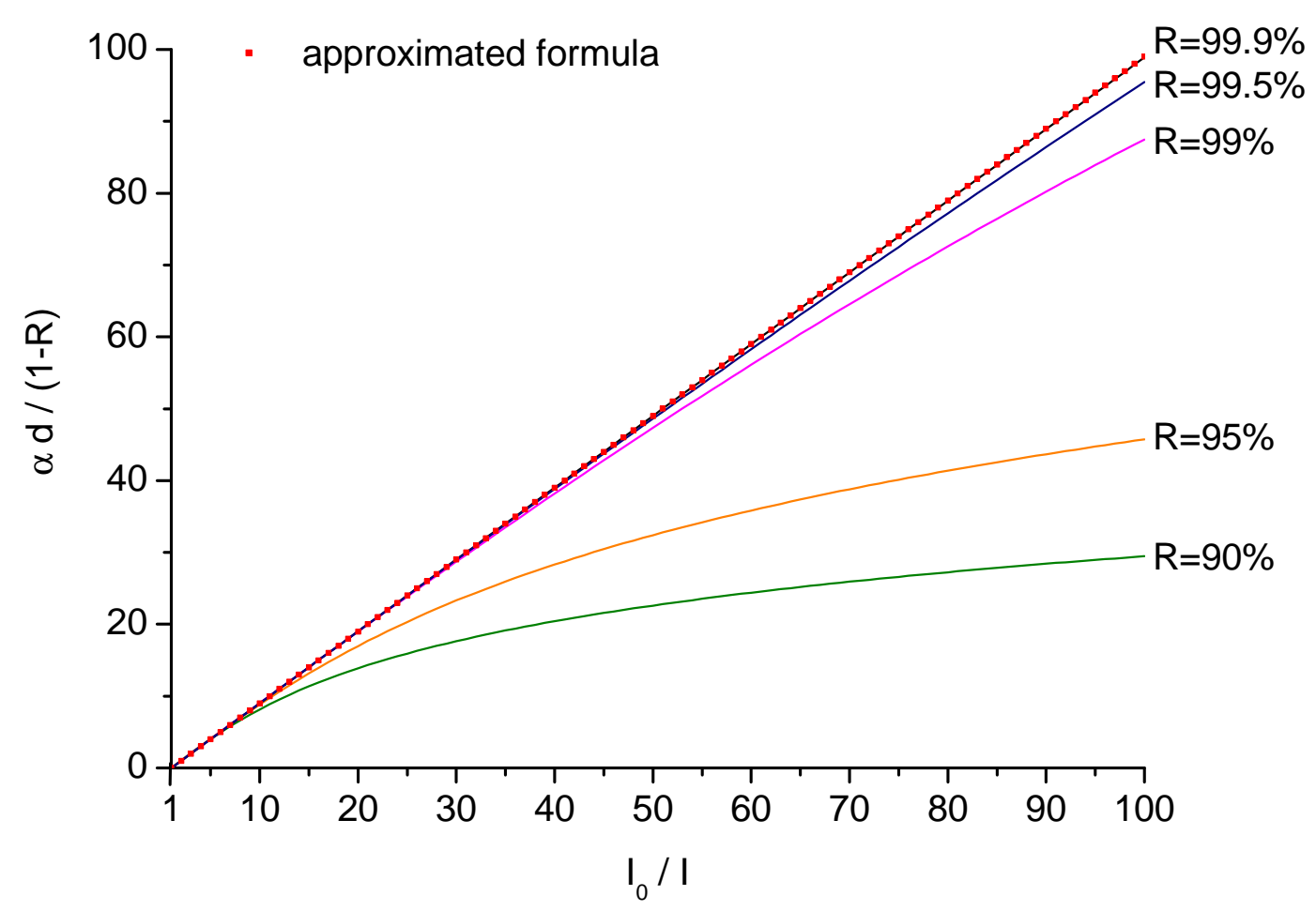

Figure 2.3: Absorption per pass (absorbance $A=\alpha \cdot d$ ) in the unit $(1-R$ ) a as a function of $I_{0} / I$ calculated for different reflectivities by Eq. 2.15- solid line - and by the approximated formula of Eq. 2.16- dotted line.

are assumed, the above equation demonstrates (setting $R=R_{1}=R_{2}$ ) that the new technique is $(1-R)^{-1}$ times more sensitive than a conventional single pass absorption method because of the longer effective path length. For instance, for mirror reflectivities of $R=0.99$, for which lower lamp luminosities are sufficient for IBBCEA measurements, this corresponds to an enhancement-factor of 100 in comparison to conventional absorption experiments.

\subsubsection{The Enhancement of a Spectrometer by IBBCEAS}

Whenever a cavity is used to enhance the sensitivity of a conventional single pass absorption experiment, the signal-to-noise ratio will be affected. Considering ideal quantum noise only and equal duration of a single pass measurement in comparison 
to a cavity-enhanced absorption measurement, the signal-to-noise ratio, $S N R=$ $N / \Delta N$, depends on the number of detected photons, $N$, with $\Delta N=\sqrt{N}$. For the sake of convention we will use the measured intensity $I$ (photons/time) to represent $N$ in the dependence of the signal-to-noise ratio, even though all equations in this section were derived using $N$. Due to the fact that the mirrors in an IBBCEA experiment will dramatically lower the intensity of the transmitted light (Eq.2.12), the intensity-dependent signal-to-noise ratio will be lower than in a single pass measurement:

$$
\begin{aligned}
& S N R_{\text {IBBCEAS }}(I)=\sqrt{\frac{\left(1-R_{1}\right)\left(1-R_{2}\right)(1-L)}{1-R_{1} R_{2}(1-L)^{2}}} S N R_{\text {single }}(I)
\end{aligned}
$$

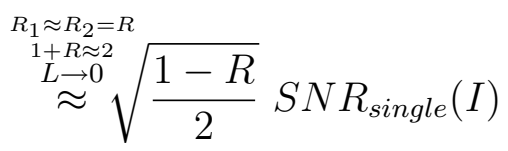

However, since for small losses the effective absorption path length in an IBBCEA measurement is increased by a factor of $\left(1-\sqrt{R_{1} R_{2}}\right)^{-1}$, the relevant signal-to-noise ratio for the absorption coefficient will be greater than in a single pass experiment. For losses, $L<(1-R)$, and mirror reflectivities $R_{1}$ and $R_{2}$, the maximum enhancement of the signal-to-noise ratio with respect to a single pass experiment is determined by

$$
\begin{aligned}
& S N R_{\text {IBBCEAS }}(\alpha)=\sqrt{\frac{\left(1-R_{1}\right)\left(1-R_{2}\right)(1-L)}{\left[1-R_{1} R_{2}(1-L)^{2}\right]\left(1-\sqrt{R_{1} R_{2}}\right)^{2}}} S N R_{\text {single }}(\alpha)
\end{aligned}
$$

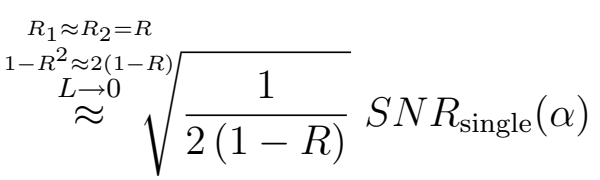

Eq. 2.21 essentially introduces a maximum enhancement factor $Q \equiv \sqrt{(2(1-R))^{-1}}$ by which the signal-to-noise ratio of measurement of $\alpha$ is improved through IBBCEAS compared to a conventional single pass absorption measurement, provided L is vir- 
tually negligible. For a mirror reflectivity of $R=0.99$ as in the example above the expected enhancement factor is $Q \approx 7$.

The situation is different in case of a system where, apart from the absorption loss of interest, $\delta L$, other significant time-independent "background losses", $L_{\mathrm{b}}$, exist, such as the scattering of a liquid or the losses introduced by the surfaces of a cuvette. In that case the overall loss $L$ is the sum of the (absorption) loss per pass, $\delta L$, and all background losses per pass, $L_{\mathrm{b}}$, which can be determined through calibration measurements, i.e. $L=L_{\mathrm{b}}+\delta L$. Let $I_{L_{\mathrm{b}}+\delta L}$ and $I_{L_{\mathrm{b}}}$ be the known intensities of the light transmitted by the cavity for the losses $L$ and $L_{\mathrm{b}}$ respectively, then the absorption coefficient, $\alpha$, associated with the absorption loss per pass, $\delta L$, can be calculated analytically (cf. Eq. 2.12):

$$
\begin{aligned}
\alpha=\frac{1}{d} \mid & \ln \left(1-L_{\mathrm{b}}+\frac{1}{2 R_{1} R_{2}}\left(\frac{I_{L_{\mathrm{b}}}}{I_{L_{\mathrm{b}}+\delta L}} \frac{\left(1-R_{1} R_{2}\left(1-L_{\mathrm{b}}\right)^{2}\right)}{1-L_{\mathrm{b}}}\right.\right. \\
& \left.\left.-\sqrt{4 R_{1} R_{2}+\left(\frac{I_{L_{\mathrm{b}}}}{I_{L_{\mathrm{b}}+\delta L}} \frac{\left(1-R_{1} R_{2}\left(1-L_{\mathrm{b}}\right)^{2}\right)}{1-L_{\mathrm{b}}}\right)^{2}}\right)\right) \mid
\end{aligned}
$$

It should be noted that again no approximations were made in order to derive Eq. 2.22). In order to find an expression for the signal-to-noise enhancement factor, $Q$, the ratio of the uncertainty $\Delta \alpha_{\text {single }}$ (=noise) of the absorption coefficient in a single pass experiment and the uncertainty $\Delta \alpha_{\text {IBBCEAS }}$ in a cavity-enhanced experiment must be calculated $Q=\left(\Delta \alpha_{\text {single }} / \Delta \alpha_{\text {IBBCEAs }}\right)$. The expression obtained for $Q$ is inexpedient, if $\Delta \alpha_{\text {IBвCEAS }}$ and $\Delta \alpha_{\text {single }}$ are derived by applying the principle of Gaussian error propagation to Eq. 2.22 and the single pass relation, $\alpha=\left(1-I / I_{0}\right) / d$, respectively. Therefore $Q$ was calculated numerically [Mat] as a function of $(1-R)$ for $R=R_{1} \approx R_{2}$, and as a function of the loss $L$ for different

$\overline{\mathbf{h} \quad S N R_{\mathrm{IBBCEAS}}(\alpha)=\frac{\alpha}{\Delta \alpha_{\mathrm{IBBCEAS}}}=Q} \frac{\alpha}{\Delta \alpha_{\text {single }}}=Q \cdot S N R_{\text {single }}(\alpha)$ 


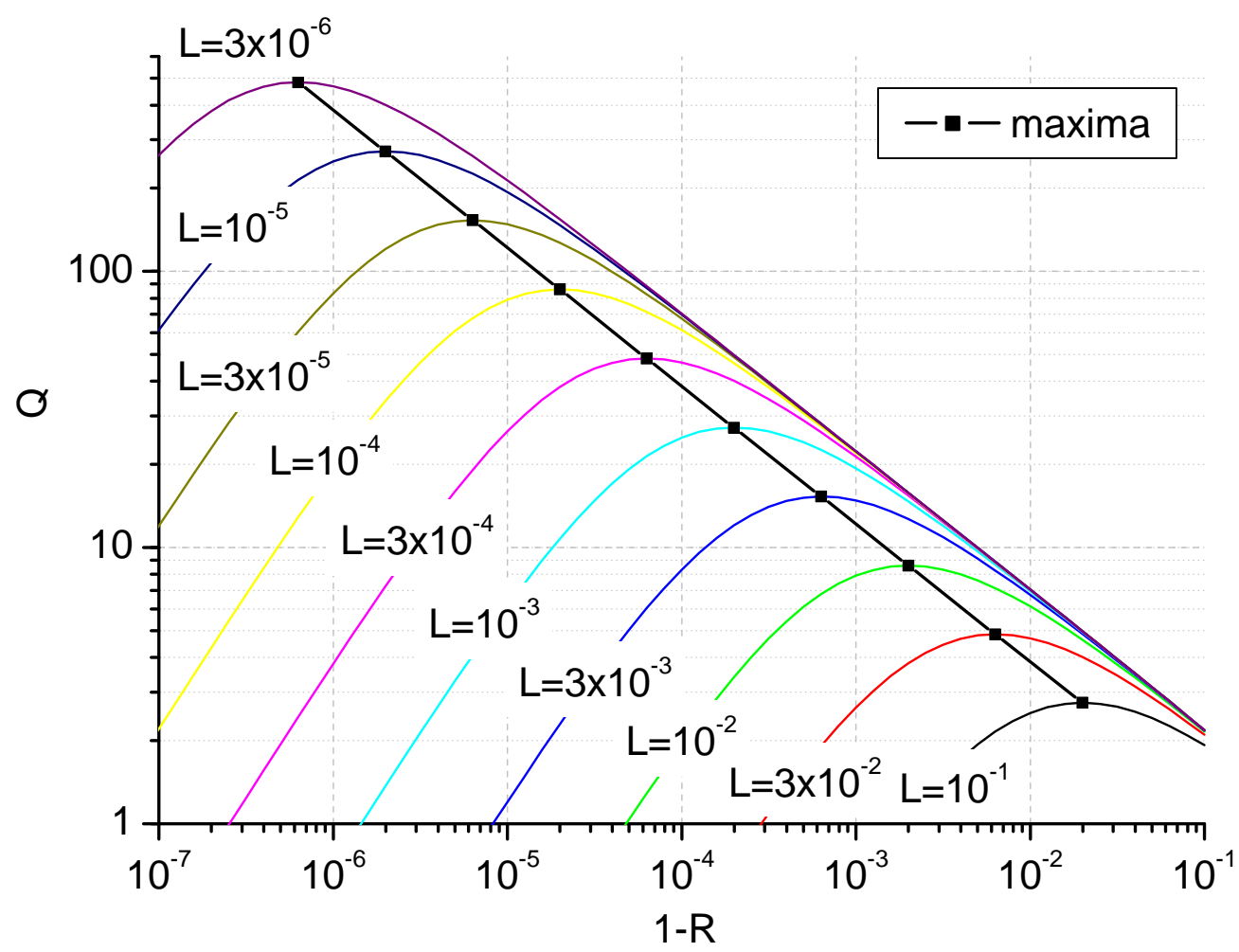

Figure 2.4: The numerically calculated signal-to-noise ratio enhancement factor $Q$ of $\overline{\text { IBBCEAS }}$ compared to a single pass experiment. In this figure $Q$ is shown as a function of $(1-R)$ for different losses $L . \delta L \rightarrow 0$ is assumed, hence $L \approx L_{\mathrm{b}}$.

reflectivities, assuming $L \approx L_{\mathrm{b}} \gg \delta L \rightarrow 0$. The results are shown in Fig. 2.4 and 2.5. For a given loss $L$, which is governed by the background losses of the system, there is an optimum value $\left(1-R_{\mathrm{opt}}\right)$ where the signal-to-noise enhancement factor is maximal, $Q_{\max }$ (see symbols in Fig. 2.4). A higher reflectivity than $R_{\text {opt }}$ for a given loss $L$ does not improve the IBBCEA experiment in comparison to a single pass measurement. The optimum reflectivities $R_{\mathrm{opt}}$ for a given loss $L_{\mathrm{b}}$ are also shown in Fig. 2.5 (straight line of symbols), where $Q$ is plotted versus $L$ for several values of $(1-R)$. If the total losses become negligibly small the upper limits of $Q$ for a given value of $(1-R)$ are approached (intercepts of the $(1-R)$-curves with the vertical axis in Fig. 2.5, compare Eq. 2.21). 


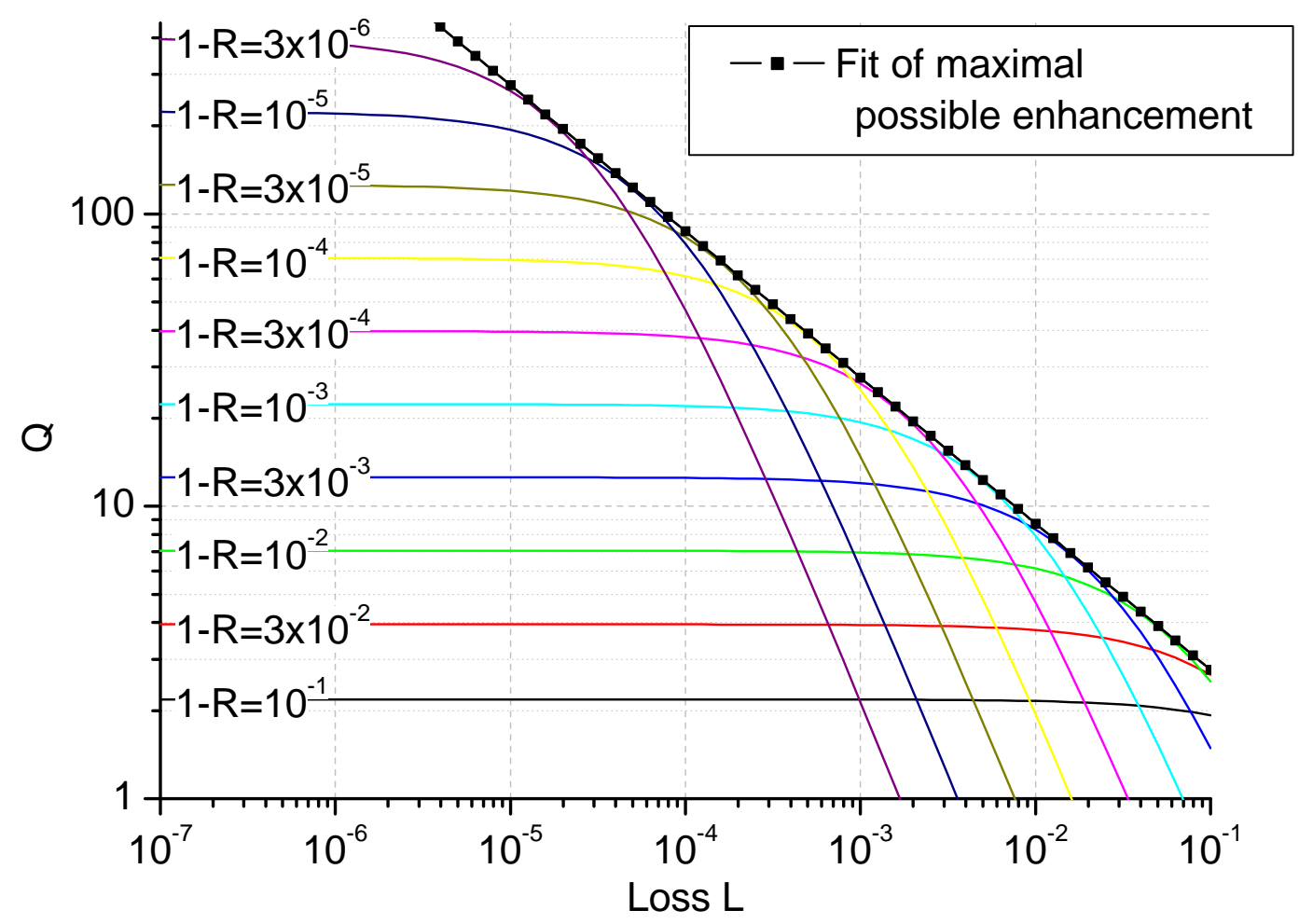

Figure 2.5: The numerically calculated signal-to-noise ratio enhancement factor $Q$ of $\overline{\text { IBBCEAS }}$ compared to a single pass experiment. In this figure $\delta L \rightarrow 0$ is assumed, hence $L \approx L_{\mathrm{b}}$, and $Q$ is shown as a function of loss $L$ for different mirror reflectivities $R$. For the measurements presented in chapter $4(\mathrm{R}=0.99)$ a maximum enhancement factor of $\approx 7$ can be achieved for small losses $\delta L \ll L_{\mathrm{b}}$.

The following power laws, $Q_{\max }=p_{1} \cdot L^{p_{2}}$, and $Q_{\max }=p_{3} \cdot(1-R)^{p_{4}}$ were fitted to the straight lines of symbols in Fig. 2.4 and 2.5 , yielding the parameters $p_{1}=0.861$, $p_{3}=0.384$ and $p_{2}=p_{4}=-1 / 2$. Hence the following simple empirical relationships between $Q_{\max }$, the background losses $L_{\mathrm{b}}(\approx L$ for $\delta L \rightarrow 0)$, and the optimum reflectivity $R_{\text {opt }}$ can be established for small absorption losses $\delta L$ :

$$
\begin{gathered}
Q_{\max }=\left(1.350 \times L_{\mathrm{b}}\right)^{-\frac{1}{2}}=(6.766 \times(1-R))^{-\frac{1}{2}} \\
1-R_{\mathrm{opt}}=\frac{L_{\mathrm{b}}}{5.012}
\end{gathered}
$$


Note that equations 2.23 and 2.24 only concern Lambert-Beer absorption losses, assuming $1-R=T$ ( $T=$ transmission) to be valid for the cavity mirrors. Additional loss factors of the mirrors, typical for the UV region below $300 \mathrm{~nm}$, are not accounted for. Saturation effects at very strong absorptions or possibly other nonlinear processes are also not considered.

For all calculations presented here, the light was regarded to be on the optical axis only. Actually, the equations presented in this section are valid even for off axis light, supposing that all of the light focussed by the lenses could be coupled without losses into the resonator and from there through the entrance slit of the spectrometer. This, however, can not always be accomplished. Due to geometric reasons not all of the light fits through the aperture of the mirrors and the light inside of the cavity could leave it sideways. Therefore light losses may be caused by long resonators, small mirror diameters, large mirror curvatures, a large light emitting area of the lamp or large divergence of the light.

Additionally the geometry of the light sent in is destroyed, due to multiple reflections in the cavity and lens effects by the concave mirror glass substrates. In other words, the light sent in has different properties than the light going out. The intensity distribution of output light is the superposition of light beams after different numbers of reflections. Compared to a single pass this may result in a larger focus at an other distance from the second lens. Therefore additional light loss at the entrance slit of the spectrometer is possible.

The optimum geometry of the lenses is quite complex to calculate and was therefore empirically assessed in this work by trial and error for each geometry used. A systematical analysis theoretically as well as experimentally will be future work (see chapter 7). 


\subsubsection{Practical Implications}

Measurements based on the IBBCEA method are intensity dependent, which has the following practical consequences:

(A) In order to measure absolute absorption cross-sections a calibration is required, or alternatively the mirror reflectivities must be known over the entire wavelength range used for the measurement.

(B) A high spectral luminosity of the light source is required in order to achieve a large signal-to-noise ratio.

(C) Due to the use of a CCD or photodiode-array, measurements of $I$ and $I_{0}$ are generally independent of intensity fluctuations of the excitation light. However, intensity variations in the separate measurements of $I$ and $I_{0}$ generally determine the uncertainty with which a baseline can be determined. In many cases however, where at certain wavelengths in the measured spectrum there is no absorption, the intensities $I$ and $I_{0}$ can be normalized and hence changes in the lamp intensity can be eliminated. In an ideal setup $I$ and $I_{0}$ should be measured simultaneously in a two-beam experiment to remove the dependence on intensity fluctuations.

(D) The spectral resolution of the technique is in principle limited by the density of eigenmodes of the cavity (free spectral range of the cavity $F S R=\frac{1}{2 d}$ ), which for all practical purposes is so high, that the resolution is ultimately limited by the resolving power of the monochromator used.

(E) Due to a limited detection sensitivity generally longer integration times are required for high resolution measurements.

It is important to stress that if the IBBCEA method is applied with mirrors of appropriate reflectivity, there is practically no upper limit for strong absorptions 
as opposed to cavity ring-down spectroscopy, where for strong absorption losses the ring-down transient becomes too short in order to be easily measurable.

As opposed to multi-pass cells White42, Herriot65, White76] in IBBCEAS always the same volume is sampled. Therefore it can be used in certain experiments with inhomogeneous distribution of the sample such as supersonic jets and flames. The size of an IBBCEAS experiment can be substantially smaller than multi-pass applications because it is not necessary to separate light spots on the mirrors. A general comparison of the signal-to-noise ratio between IBBCEAS and (Whiteor Herriott-type) multi-pass cells is not possible since the number of passes in IBBCEAS depends solely on the mirror reflectivity (and losses in the sample) as opposed to the set-up geometry and f-number of the light imaging optics in case of a multi-pass cell. Generally the effective path length which can be achieved with IBBCEAS is longer than in multi-pass absorption cells, it can however not be controlled through the cavity's geometry (only by using different mirror reflectivities).

In the calculations concerning the signal-to-noise enhancement ideal quantum noise was assumed. Real detectors however are sources of "digital" as well as "dark" noise, which can affect the measurement. If the transmitted light intensity of a single pass measurement is very high, the detector is ultimately limited by the A/D converters resolution and digital noise can be introduced in form of jumps between two digits. In such a case an additional improvement of the signal-to-noise ratio with IBBCEAS can be expected, because the intensity is reduced by the cavity mirrors and quantum noise becomes dominant again. If however the intensity is reduced too much by the cavity mirrors, the dark noise of the detector dominates and the enhancement of the signal-to-noise ratio will be smaller than calculated in this section. 


\section{Chapter 3}

\section{Oxygen and Azulene in the Gas Phase}

Simple and sensitive techniques to measure broad-band absorptions of gaseous samples are of high interest not only for applied atmospheric and exhaust sciences but also for fundamental research. In this section the features of IBBCEAS are demonstrated for gas cells exemplary at the broad absorption bands of gaseous azulene and at the narrow and very weak absorption lines of oxygen.

\subsection{Experimental Setup}

The experimental apparatus of IBBCEAS for the gas phase measurements is schematically shown in Fig. 3.1. The light source is a short-arc xenon lamp (ISAS Berlin) with a specified luminosity of $18 \mathrm{~W} \mathrm{~cm}^{-2} \mathrm{sr}^{-1} \mathrm{~nm}^{-1}$ at $400 \mathrm{~nm}$. The lamp current is stable to within $2-3 \%$. The light intensity is additionally subject to fluctuations of up to $\sim 10 \%$, if random spatial jumps of the light-arc occur. These variations can however be accounted for in the evaluation procedure. The white 


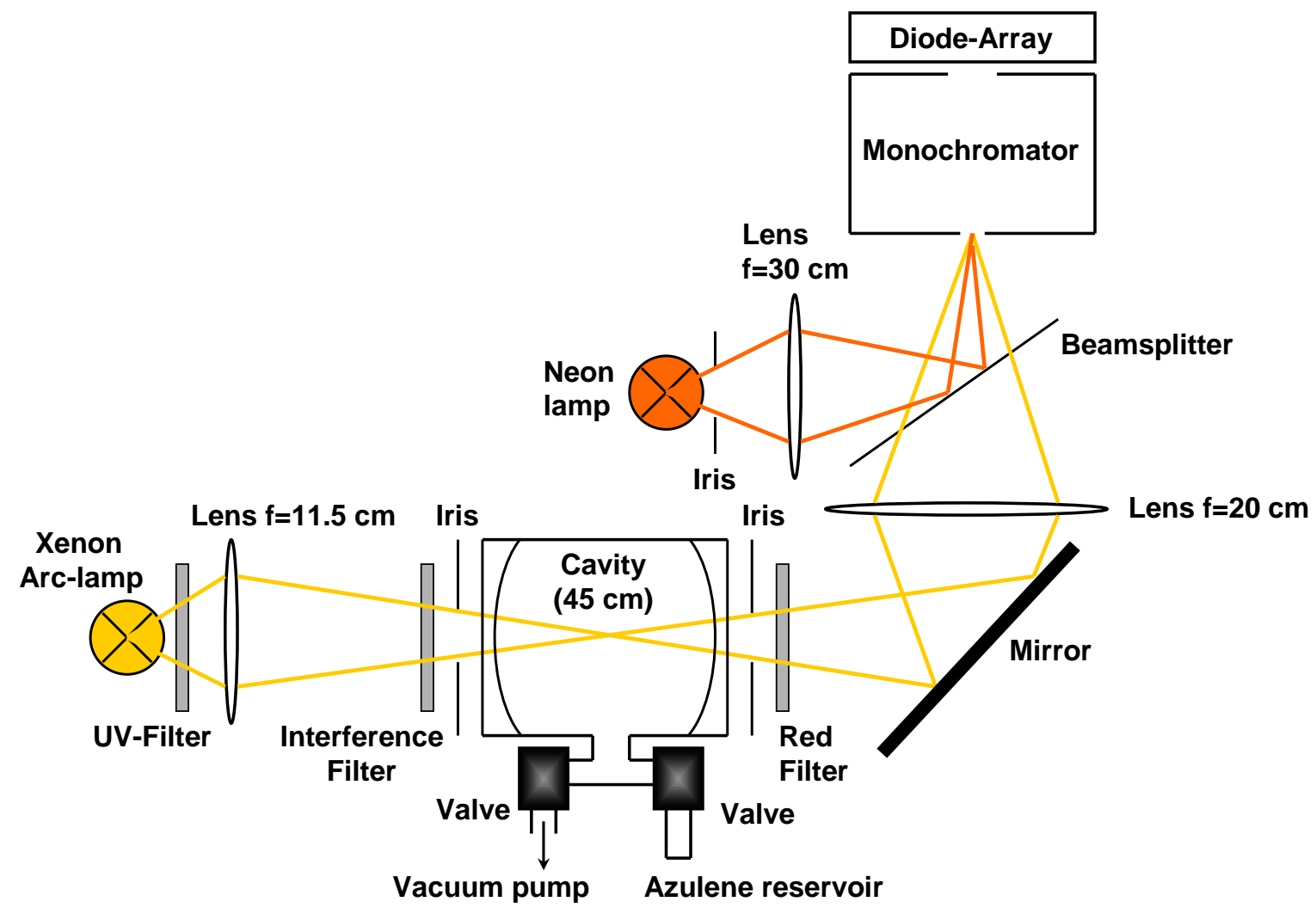

Figure 3.1: Experimental setup schematically for IBBCEAS of gaseous samples. The optical filters used depend on the region of high reflectivity of the cavity mirrors and their position in the setup is irrelevant, since they only reduce unwanted scattered (and second order) light in the monochromator. The neon-lamp is used for wavelength calibration only. The light guide before the entrance slit of the monochromator is not shown.

light is narrowed by an interference filter to the spectral range, which corresponds to the high reflectivity wavelength region of the dielectric mirrors forming the optically stable cavity. The reflectivity of the mirrors (REO, Boulder Colorado) at the center wavelength of $610 \mathrm{~nm}$ is $R>0.9999$, the curvature is $|r|=200 \mathrm{~cm}$, and the length of the cavity is $\ell=d=45 \mathrm{~cm}$. A set of lenses images the brightest zone of the arc lamp $(\sim 150 \mu \mathrm{m})$ through the mechanically not stabilized cavity as shown in Fig. 3.1. Before the light of the lamp enters the cavity it passes through a UV cut-off filter (335 nm) to suppress light of wavelengths around $305 \mathrm{~nm}$, which 
corresponds to the 2 nd order of a grating. The two mirrors of the cavity are mounted to a vessel, which was evacuated, flushed with an inert gas or filled with the sample gas. Two irises on either side of the cavity are to spatially define the light beam and avoid transmission of transverse modes of high order. The light transmitted by the cavity is coupled into a light-guide with circular input aperture and rectangular output aperture, which is connected to the entrance slit of a grating monochromator. For the measurements with oxygen a $1.5 \mathrm{~m}$ Jobin-Yvon monochromator (2400 grooves $/ \mathrm{mm}$ ) with spectral resolution of $\sim 0.026 \mathrm{~nm}$ was used to disperse the light; for azulene a small $0.25 \mathrm{~m}$ B\&M Spektronik monochromator (600 grooves $/ \mathrm{mm}$ ) with low spectral resolution of $\sim 1.2 \mathrm{~nm}$ was employed. The emission from a neon-lamp, which is used for the wavelength calibration only, enters the light-guide at the same angle as the light transmitted by the cavity (see Fig. 3.1).

Assuming only losses due to the limited mirror reflectivity, the light intensity is inherently reduced by a factor of $\frac{1}{2}(1-R)$ by the resonator (confer Eq. 2.13) and further lowered by the spectral transmission of the monochromator. Therefore, a coolable and intensified photodiode-array detector with low noise level (IRY700S/BP-46, Princeton Instruments) is used, which is interfaced to a computer. By integrating and averaging the light transmitted by the cavity over time, the absorption spectrum of the sample gas filling the resonator can be obtained. At very high reflectivities $R$ the signal-to-noise ratio is ultimately limited by the dark signal of the detection system or integration times can become unreasonably long for practical purposes; these limits were however not reached in the present investigation. On the contrary, integration times were surprisingly short considering a mirror reflectivity of $\approx 0.9999$. In a measurement with a spectral resolution $<1 \mathrm{~cm}^{-1}$ (see measurement on $\mathrm{O}_{2}$ presented in subsection 3.3 ) it can be estimated that the signal of the intensified photodiode-array would reach the noise level at 
$R \approx 0.99999$. For state-of-the-art CCD detectors with lower electronic noise than the diode-array used, mirror reflectivities of 0.99999 and even slightly larger (if available) are experimentally feasible, particularly if a monochromator with a low dispersion is employed.

\subsection{IBBCEAS of Molecular Oxygen}

The $\mathrm{b}^{1} \Sigma_{g}^{+}\left(v^{\prime}=2\right) \leftarrow X^{3} \Sigma_{g}^{-}\left(v^{\prime \prime}=0\right)$ triplet-singlet transition of molecular oxygen is very weak, because it is doubly forbidden by quantum mechanics. The symmetry of this transition, $\Sigma_{g}^{+}-\Sigma_{g}^{-}$, allows the absorption of magnetic dipole radiation only and the selection rule for the spin conservation is violated, which causes a reduction of the cross section of the order of $10^{3}$. Additionally the second vibronic transition $\left(v^{\prime}=2\right)$ is $\sim 300$ times weaker than the origin $\left(v^{\prime}=0\right.$, cp. Engeln96), due to Franck-Condon inhibition. Therefore and because of the availability of oxygen this transition is widely used as a standard test for high sensitive absorption experiments like CRDS or in this case IBBCEAS.

Fig. 3.2 shows part of the $\mathrm{b}^{1} \Sigma_{g}^{+}\left(v^{\prime}=2\right) \leftarrow X^{3} \Sigma_{g}^{-}\left(v^{\prime \prime}=0\right)$ transition from 15865 to $15930 \mathrm{~cm}^{-1}$ in molecular oxygen at 1000 mbar at room temperature measured with IBBCEAS. The spectrum was obtained by measuring the intensity ratio $\left(I_{\mathrm{O}_{2}}-\right.$ $\left.I_{d}\right) /\left(I_{N_{2}}-I_{d}\right)\left[\equiv\left(I / I_{0}\right)\right.$ in Eq. 2.16], where $I_{O_{2}}$ and $I_{N_{2}}$ are the transmitted intensities with only oxygen and only nitrogen in the cavity, respectively. $I_{d}$ is the dark signal measured with the light beam blocked and was like $I_{N_{2}}$ measured only once. The $\alpha$-axes were obtained using Eq. 2.16. The spectral resolution for the IBBCEA spectrum is $0.66 \mathrm{~cm}^{-1}(\approx 0.026 \mathrm{~nm}$ in that wavelength region) and it was measured by averaging 100 spectra taken with an exposure time of $5 \mathrm{~s}$ each 


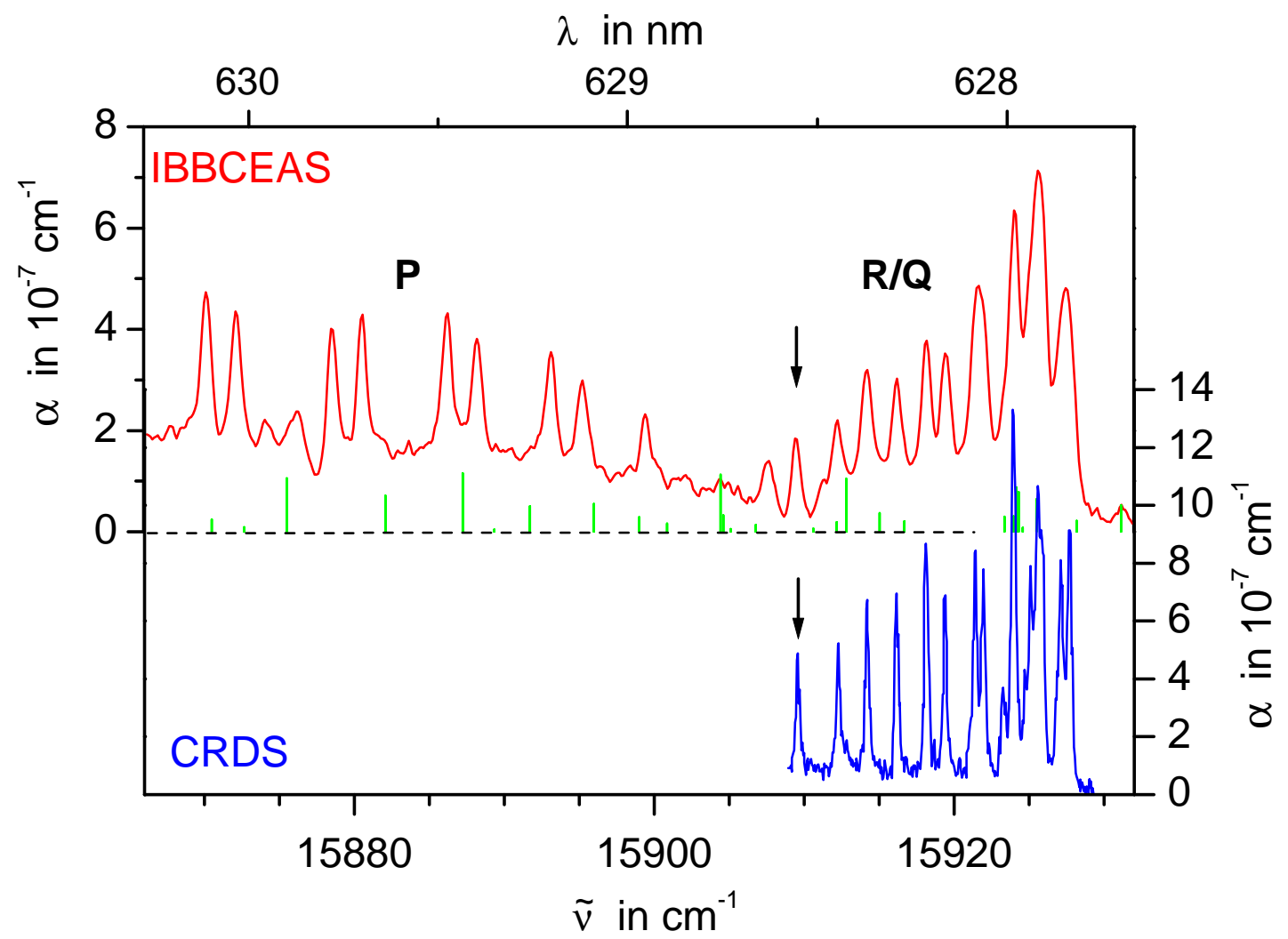

Figure 3.2: Absorption spectrum of the $\mathrm{b}^{1} \Sigma_{g}^{+}\left(v^{\prime}=2\right) \leftarrow X^{3} \Sigma_{g}^{-}\left(v^{\prime \prime}=0\right)$ transition (Q/R and part of $\mathrm{P}$ branch) in molecular oxygen at 1000 mbar in a static cell at room temperature measured with IBBCEAS and pulsed CRDS.

(total exposure time $500 \mathrm{~s}$ ). The rovibronic lines in the low energy $\mathrm{P}$ branch ${ }^{\mathrm{a}}$ are spectrally separated. The increased background may be due to an incomplete compensation of the decreasing mirror reflectivity in this region of the spectrum. Also water, which may have been present in the cavity, may contribute to the background signal. Relative absorption strengths and position of water absorptions according to the HITRAN Hitran98] database are indicated by green bars at the middle of Fig. 3.2 .

a The rotational structure of an electronic transition are divided in branches, called $\mathrm{P}$ if the rotational quantum number $J$ changes by $\Delta J=-1$, Q if $\Delta J=0$ and $\mathrm{R}$ if $\Delta J=+1$. 
The unresolved band head (the $\mathrm{Q}$ and $\mathrm{R}$ branches) was also measured in the same cavity by pulsed CRDS also shown in Fig. 3.2. The spectrum was measured using a grazing incidence dye laser (1800 lines/mm and DCM as dye) pumped by the 2nd harmonic of a Nd:YAG laser (Model HY 500, Fa. Lumonics, JKL GmbH), an Hamamatsu R 1477 photomultiplier and a LeCroy 9450A 300 Mhz Dual channel oscilloscope. The pulsed laser achieved a spectral resolution of $0.07 \mathrm{~cm}^{-1}$ [?]. The usual cavity ring down times were $\tau_{\mathrm{CRD}} \approx 22 \mu$ s. Hence the absorption coefficients $\alpha$ are somewhat larger than in the IBBCEA spectra. The stepsize in the CRD spectrum is $0.06 \mathrm{~cm}^{-1}$ and the overall time to record the spectrum was $\sim 4500 \mathrm{~s}$, using 150 samples per datapoint at a $20 \mathrm{~Hz}$ laser repetition rate. This demonstrates the significantly improved time resolution of IBBCEAS in comparison to standard pulsed CRDS. In order to directly compare the measurements in Fig. 3.2 with the literature we integrated the ${ }^{R} \mathrm{Q}(1)$ line at $15909.5 \mathrm{~cm}^{-1}$ (indicated by vertical arrows) and compared it to the intensity of $7.351 \times 10^{-27} \mathrm{~cm} /$ molecule given in the HITRAN database for $296 \mathrm{~K}$ [Hitran98]. The IBBCEA and CRD measurements yielded values of $6.8 \times 10^{-27}$ and $8.7 \times 10^{-27} \mathrm{~cm} /$ molecule, respectively, which is in good agreement with the literature value considering the uncertainties regarding the integration limits, the background of the IBBCEA spectrum and and stepsize of the CRD spectrum. The oxygen absorption spectrum can also be compared with a Fourier transform spectrum taken in a long-path $(\approx 257 \mathrm{~m})$ measurement of 1000 mbar oxygen [Newnham98]. The signal-to-noise ratio in Fig. 9 of Ref. [Newnham98] is significantly smaller in comparison to our measurement. The range of cross-sections $\left(\sim 13 \times 10^{-26} \mathrm{~cm}^{2}\right)$ shown there is in satisfactory agreement with the one presented here $\left(\sim 3 \times 10^{-26} \mathrm{~cm}^{2}\right)$ considering the higher resolution and the error limits stated in Newnham98. Through Fig. 3.2 it is also demonstrated that the sensitivity of IBBCEAS is comparable to that of CEAS (see Fig. 3 in Ref. Engeln98), the main difference is the spectral resolution. 


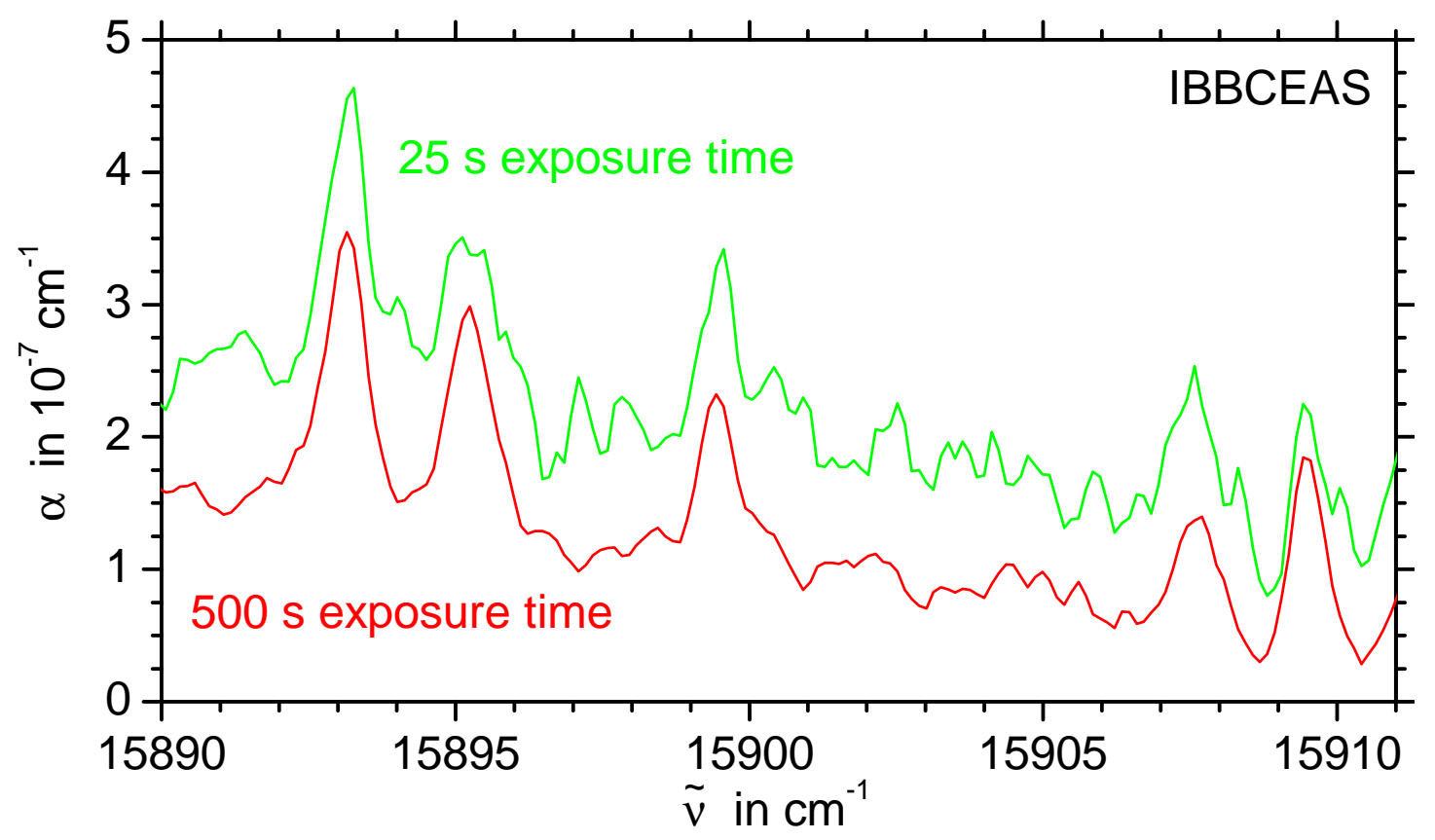

Figure 3.3: Comparison of two oxygen spectra measured by IBBCEAS with $25 \mathrm{~s}$ (green) and $500 \mathrm{~s}$ (red) exposure time.

\section{Influence of Exposure Times on the Signal-to-Noise Ratio}

In Fig. 3.3 the effect of different exposure times for IBBCEAS measurements is demonstrated. The upper spectrum was measured by averaging 5 spectra taken with an exposure time of $5 \mathrm{~s}$ each (total exposure time $25 \mathrm{~s}$ ). For the lower spectrum number the total exposure time was extended by a factor 20 to $500 \mathrm{~s}$ by averaging 100 spectra. An improvement by a factor $\sqrt{20} \approx 4.5$ in the signal-tonoise ratio is therefore to be expected. However, the experimental signal-to-noise ratio of $\sim 25$ for the longer exposure time is only by factor $\approx 2$ better than for the shorter exposure time. This discrepancy can be explained by a relatively high dark noise of the detector. The dark counts of the detector already added up to a quarter of the measured intensity. They were measured separately and subtracted from the intensity signal. The dark noise however can not be reduced by the sub- 
traction of the dark counts. Since the dark noise depends on the detector and does not act in accordance with quantum statistics, longer exposure times do not decrease the dark noise in the same manner like the quantum noise is diminished. Therefore a detector with very low dark noise, like for instance provided by modern cooled CCD-arrays, would be more appropriate for very sensitive absorption measurements.

\section{Summary}

By measuring the weak $\mathrm{b}^{1} \Sigma_{g}^{+}\left(v^{\prime}=2\right) \leftarrow X^{3} \Sigma_{g}^{-}\left(v^{\prime \prime}=0\right)$ triplet-singlet transition of molecular oxygen the incoherent broad-band cavity-enhanced absorption spectroscopy proofed to be a high sensitive absorption technique. Using a resolution of $0.66 \mathrm{~cm}^{-1}(\approx 0.026 \mathrm{~nm})$ a sensitivity of $3 \times 10^{-8} \mathrm{~cm}^{-1}$ for the absorption coefficient could be achieved. The narrow observed spectral region (only $2.5 \mathrm{~nm} \approx 65 \mathrm{~cm}^{-1}$ ) is due to the setup of the monochromator and the photodiode-array and not a problem of principle. Other monochromator types (e.g. Echelle-monochromators [Becker-Ross97]) will be able to cover the whole high reflectivity range of the used dielectric mirrors with even higher resolution.

\subsection{IBBCEAS of Gaseous Azulene}

The $\mathrm{S}_{1} \leftarrow \mathrm{S}_{0}$ transition of azulene $\left(\mathrm{C}_{10} \mathrm{H}_{8}\right)$ shows at room temperature broad vibronic structures and the extraordinarily fast non-radiative relaxations (see chapter 6) make fluorescence measurements almost impossible. Therefore the $\mathrm{S}_{1}$ manifold is simplest studied in a direct absorption experiment, however, the low oscillator strength of $f \sim 0.009$ [Hunt62] requires a sensitive absorption method. 


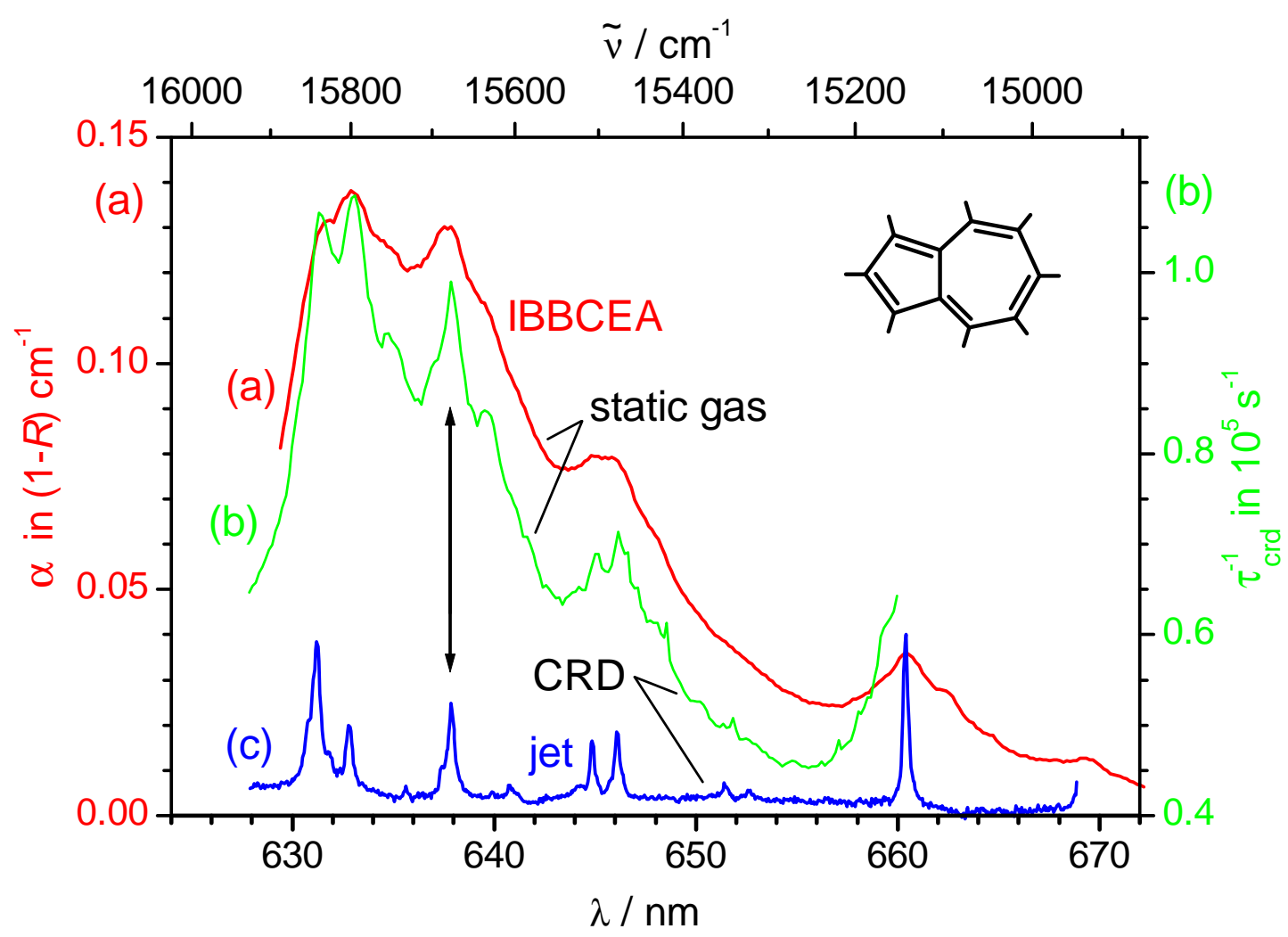

Figure 3.4: $\quad \mathrm{S}_{1} \leftarrow \mathrm{S}_{0}$ absorption spectrum of gaseous azulene in a static cell at room temperature measured with IBBCEAS (trace (a), left axis) and pulsed CRDS (trace (b), right axis). The lower trace ((c) in arbitrary units) is a spectrum of azulene in a cold pulsed supersonic jet, which was also measured by CRDS [Ruth99].

Fig. 3.4 shows part of the $\mathrm{S}_{1} \leftarrow \mathrm{S}_{0}$ absorption spectrum of gaseous azulene in a static cell at room temperature measured with IBBCEAS (a) and with pulsed CRDS (b) in the region between 628 and $670 \mathrm{~nm}$, where several vibronic transitions are located [Ruth99]. On the left axis of Fig. 3.4. referring to the IBBCEA measurement (a) with an exposure time of $\approx 90 \mathrm{~s}$, the absorption is expressed in terms of $\alpha$ given in units of $\left[(1-R) \mathrm{cm}^{-1}\right]$ for a resonator of $d=45 \mathrm{~cm}$ (confer Eq. 2.16). This illustrates that generally not the absolute value of $\alpha$ is determined by IBBCEAS, but an absorption coefficient that refers to the wavelength dependent reflectivity $R(\lambda)$ of the mirrors, which was not exactly known for the 
azulene measurements shown in this section. For IBBCEA measurements either $R(\lambda)$ must be known from an independent experiment, or the spectrum must be calibrated with a species of known absorption or concentration. For measurements of spectra (a) and (b) different chambers were used and different amounts of azulene were present in the optical cavity, owing to the very long equilibration time of the sublimation process; partial pressures were however not determined. Assuming $R=0.9999$ the relative concentrations (a) : (b) were however estimated by the absorption coefficients in the spectra $\left(\alpha_{a} \approx 1.3 \times 10^{-5}, \alpha_{b} \approx 2.0 \times 10^{-6}\right.$ and $\alpha_{c} \approx 1.4 \times 10^{-7} \mathrm{~cm}^{-1}$ ) to be $1: 0.15$ at $637.8 \mathrm{~nm}$.

The right axis in Fig. 3.4 refers to the static cell CRD experiment (b) in units of the reciprocal value of the measured ring-down time $\tau_{c r d}$. Thus spectrum (b) is also expressed relative to a certain unspecified mirror reflectivity "baseline". This way of displaying spectrum (b) was chosen in order to allow a better comparison with the IBBCEA spectrum (a). Since the mirror reflectivity decreases significantly over the range of the spectra (a) and (b), the absorption at long wavelengths is somewhat too small. This fact can be seen in comparison with the pulsed CRD absorption spectrum of jet-cooled azulene (c), which is corrected (in arbitrary units) regarding the mirror reflectivity (see [Ruth99]). In the CRD jet-spectrum (c) the features at $\sim 631$ and $\sim 660 \mathrm{~nm}$ are approximately equally strong, which is not the case in the spectra (a) and (b). This demonstrates the significant decrease of the mirror reflectivity at longer wavelengths.

The spectral resolution in the CRD spectrum (b) is due to the interval at which the dye laser was stepped in this experiment, i.e. $\sim 0.15 \mathrm{~nm}$. The spectral resolution in the IBBCEA measurement (a) is significantly lower, i.e. $\sim 1.2 \mathrm{~nm}$. This demonstrates that the IBBCEA spectrum is not fully resolved, because the CRD spectrum (b) exhibits significantly more structure. All main features are nevertheless observed in the IBBCEA spectrum, despite the $\approx 10$ times lower resolution. 
Since the IBBCEA spectrum (a) was taken at room temperature, there is a significant population of rovibrational ground states leading to the observed strongly broadened rovibronic contour bands. Comparison of the static cell spectra (a) and (b) with the absorption spectrum of jet-cooled azulene (c) demonstrates this effect of temperature on the spectrum.

\section{Summary}

The broad-band capabilities of IBBCEAS were demonstrated by measuring the $\mathrm{S}_{1} \leftarrow \mathrm{S}_{0}$ absorption spectrum of gaseous azulene in a static cell at room temperature. Using a monochromator resolution of $1.2 \mathrm{~nm}$ a wavelength region of $44 \mathrm{~nm}$ was measured broad-band, which is almost the full high reflectivity region of the mirrors used. This measurement showed also, that it is vital for IBBCEAS to know the exact wavelength dependence of the mirror reflectivities. 


\section{Chapter 4}

\section{Benzene in the Liquid Phase}

Although cavity ring-down spectroscopy (CRD) is a well established sensitive technique only recently a few approaches were made to measure absorptions in the liquid phase with CRD spectroscopy [Xu02, Hallock02, Hallock03, Snyder03, Tong03]. Due to high scattering losses in liquids ring-down transients are generally rather short $(\sim \mathrm{ns})$ and therefore challenging to measure. The noise of the spectra is increased by density fluctuations and striae in the liquid. IBBCEA spectroscopy is not only able to measure high absorptions but also avoids the fluctuation problems by averaging the whole spectrum at the same time through a broad-band measurement.

In this chapter the application of incoherent broad-band cavity-enhanced absorption spectroscopy (IBBCEAS) to weak transitions of liquid benzene through a very straightforward modification of a commercially available double-beam UV/VIS absorption spectrometer is reported.

Due to strong inhomogeneous broadening of absorption features in solution a high resolution is generally not required and a broad-band technique is predestined for such measurements. Loss fluctuation problems are avoided by averaging an entire 
spectrum at the same time and measurement times can be kept reasonably short due to broad-band detection and signal integration.

Benzene $\left(\mathrm{C}_{6} \mathrm{H}_{6}\right)$ is a transparent solvent with general optical losses of the order of $\left(\sim 10^{-3} \mathrm{~cm}^{-1}\right)$ in the visible spectrum (conservative estimate based on our measurement). Apart from a strong absorption "edge" owing to the electronic $\mathrm{S}_{1} \leftarrow \mathrm{S}_{0}$ transition with onset in the near UV at $\sim 278 \mathrm{~nm}$, benzene also exhibits weak absorption features at 541 and $607 \mathrm{~nm}$ due to vibrational overtones of the molecule in $\mathrm{S}_{0}$ (Franck-Condon inhibited absorption of the sixth and fifth C-H stretch overtone [Reddy82]). These weak absorption bands of pure benzene were used to demonstrate the superior signal-to-noise ratio of IBBCEAS in comparison to single pass absorption spectroscopy.

A straightforward way to perform an IBBCEA experiment with liquid samples is to fill the solvent/solution directly into the volume between the mirrors of the cavity. However, the reflectivity of the mirrors depends on the difference of the refractive indices of the boundary layer of the dielectric coating and air. Owing to the fact that liquids possess significantly larger refractive indices than air, the reflectivities of the mirrors are therefore reduced in such an approach. Moreover, since the reflectivities must be known in order to determine absolute absorption coefficients, the direct contact of liquid and mirror coating is particularly inappropriate and should generally be avoided. A promising and experimentally simpler implementation is placing a liquid-filled optical cuvette into the cavity. For such a setup the effect of the cuvette windows on the overall losses must be considered. 


\subsection{Cuvette Windows in a Cavity}

A typical transparent cuvette window causes additional losses in the resonator: (1) Absorption losses in the window material, which depend on the corresponding absorption coefficient, the thickness of the window and the angle of incidence, but not on the polarization of the light. (2) Reflection/scattering losses at the surfaces, which depend on the surface roughness, the angle of incidence and the polarization.

The cavity used for this experiment had a length of $\ell=197 \mathrm{~mm}$, a diameter of $\varnothing 22 \mathrm{~mm}$, reflectivities of $R \simeq 0.99$ and a curvature of the mirrors of $|r|=200 \mathrm{~mm}$. The losses of a BK7 glass plate (flatness $\lambda / 4$ ) inside the cavity were measured as a function of the angle of incidence, $\theta$, by gradually tilting the plate towards the optical axis of the resonator. For this measurement the light was polarized in front of the resonator perpendicular to the rotation axis of the glass (Brewster arrangement). Fig. 4.1a shows the result for different angles of incidence. In addition to the expected minimum at the Brewster angle $\left(\theta_{b}=\arctan (n)=56.5^{\circ}\right.$ for BK7) according to the Fresnel formula [Born80],

$$
R_{\|}=\frac{\tan ^{2}\left(\alpha-\arcsin \left(n_{1} / n_{2} \cdot \sin \alpha\right)\right)}{\tan ^{2}\left(\alpha+\arcsin \left(n_{1} / n_{2} \cdot \sin \alpha\right)\right)}
$$

a second minimum is found for perpendicular incidence of light. For $\theta=0^{\circ}$ the light is simply reflected back by the glass plate and therefore remains in the cavity. This loss minimum is quite narrow compared to the "Brewster minimum". Consequently, minimizing losses at $\theta=0^{\circ}$ requires a more accurate angular alignment of the plate in the cavity than at the Brewster angle ${ }^{\mathrm{a}}$.

In IBBCEAS the light rays coupling into the cavity have a certain angular distri-

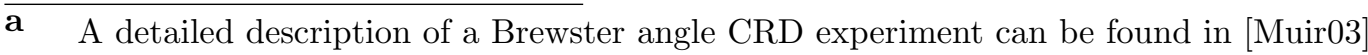




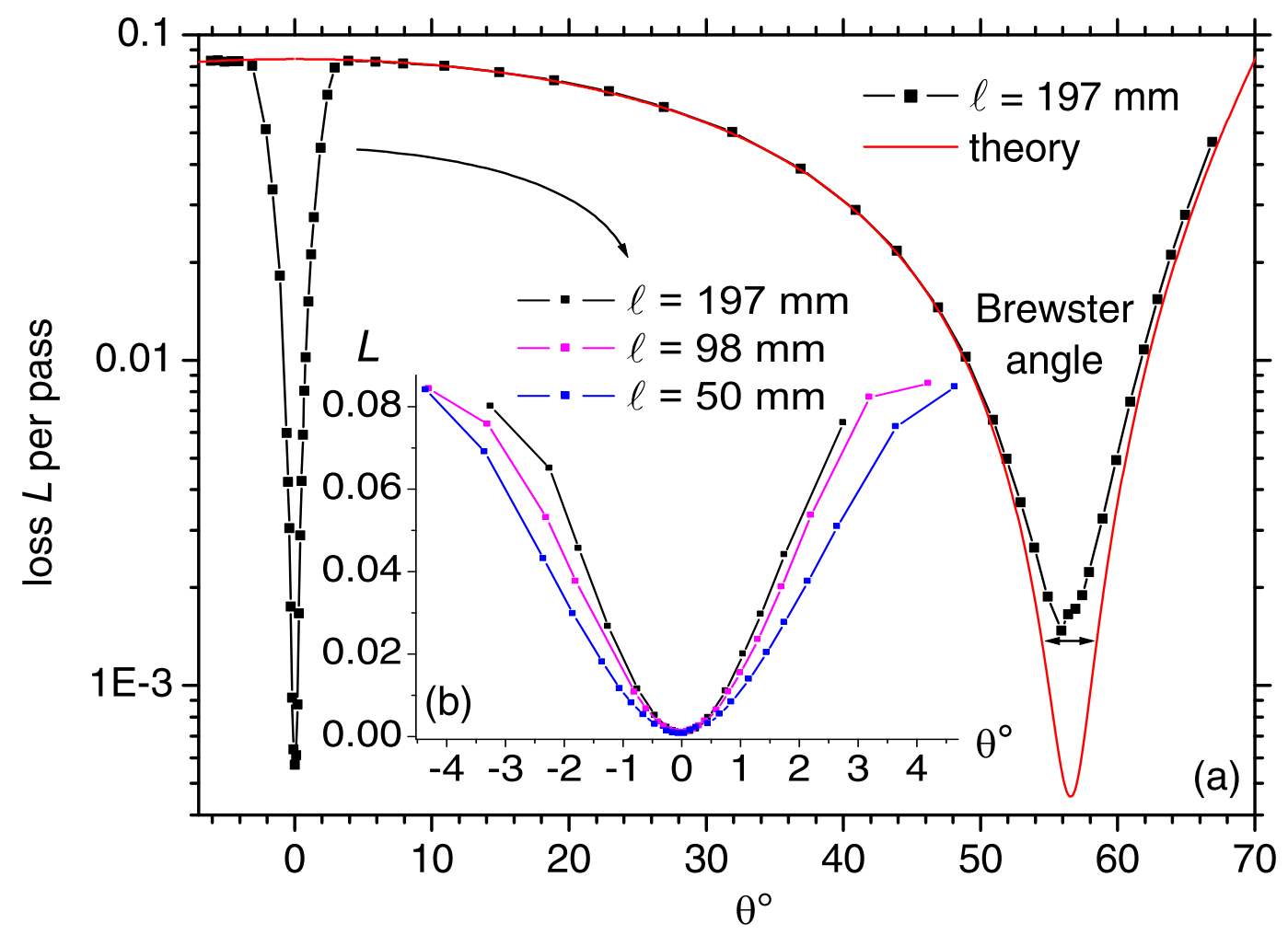

Figure 4.1: Losses induced by a BK7 glass plate (flatness $\lambda / 4$ ) in an optical resonator $\overline{\text { (length } \ell}=197 \mathrm{~mm}$ ) for polarized light (logarithmic loss scale), measured with the IBBCEA method for different angles of incidence $\theta$. Dotted lines: Measured losses per pass. Red line: Calculated loss curve according to Fresnel equation taking the inherent absorption of BK7 into account. Insert: The loss minimum around $\theta=0^{\circ}$ for different resonator lengths (linear loss scale).

bution with respect to the optical axis and hence the glass window. The angular distribution is predetermined by the angular entrance aperture of the cavity (for our cavity configuration the maximal possible angles were $\pm 6.5^{\circ}$ ) and causes the Brewster angle to be ill-defined in the experiment. Therefore the measured loss of $(14 \pm 1) \cdot 10^{-4}$ at $\theta=56^{\circ}$ is significantly higher than the pure absorption loss of a BK7 glass plate at the Brewster angle, which should only be $(4.5 \pm 0.8) \cdot 10^{-4}$ (thickness: $1.6 \pm 0.1 \mathrm{~mm}$ ) according to the Schott glass catalogue [Schott04]. From the measured minimum and the width of the theoretical curve (marked with an 
arrow) an average angle of $1.9^{\circ}$ of the light rays with respect to the optical axis can be estimated.

The stability geometry of the cavity determines in how far narrow angular distributions of the rays coupling into the cavity can be compensated. In short cavities the lateral off-set of the reflected beams is smaller for a given angle of the BK7 substrate, in other words the amount of light leaving the cavity sideways is lower. For long cavities this property is reversed. This effect is shown in the insert in Fig. 4.1 b. The width of the minimum increases as the distance, $\ell$, between the cavity mirrors is reduced.

Since the loss for the $0^{\circ}$-position of the window is only $(5.3 \pm 0.5) \cdot 10^{-4}$, which is almost as low as the pure absorption value of $(3.8 \pm 0.7) \cdot 10^{-4}$ for a BK7 glass plate, all IBBCEAS measurements on liquids were carried out in a cuvette with windows perpendicular to the optical axis of the cavity. This fact has two advantages: (i) Non-polarized light can be used for the measurement, which allows the maximum light intensity to be applied as opposed to the polarized case, where the intensity is halved. (ii) Standard cuvettes can be used for the measurements.

\subsection{IBBCEA Setup in a Spectrometer}

All but one IBBCEA measurement of benzene in the liquid phase were carried out by means of a standard double-beam UV/VIS absorption spectrometer (PerkinElmer Lambda-9 series, standard halogen lamp). In this spectrometer the monochromator is located between the excitation lamp and the sample. Therefore the wavelength distribution of the excitation light is narrow and depends on the slit width of the monochromator. The broad-band advantage is lost in such a spectrometer, because the monochromator requires slewing. However, the measurement princi- 


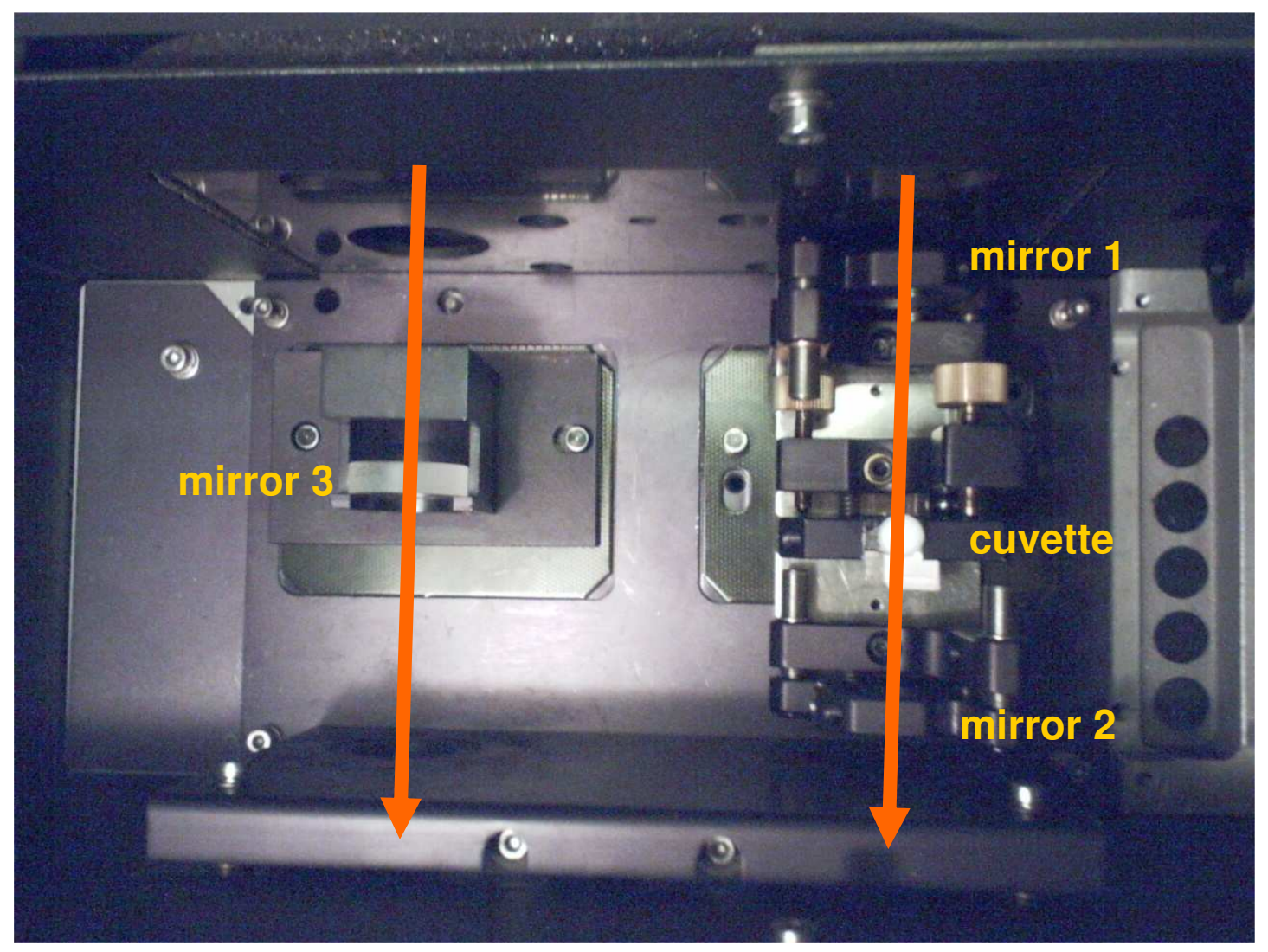

Figure 4.2: Photograph of the double-beam sample chamber of the Perkin-Elmer $\overline{\text { Lambda-9 }}$ spectrometer modified according to the IBBCEAS principle. The sample cuvette is simply placed in between two highly reflecting mirrors $R=0.99$ (right side) an additional mirror is placed in the reference beam (left side).

ple should also be applicable in conjunction with other (more modern) broad-band spectrometers which use diode arrays or CCDs to detect the transmission of the sample. An optical cavity assembly, consisting of a cylindrical sample cuvette (Hellma GmbH, $\varnothing=19 \mathrm{~mm}$, suprasil window thickness $1.25 \mathrm{~mm}$ ) in the middle between two identical, spherical, concave mirrors $(\varnothing=25 \mathrm{~mm})$ was placed in the sample beam of the Lambda-9 as shown in Fig. 4.2 $\mathrm{p}$. The mirror separation

b This experimentally very simple realization of the IBBCEA principle may significantly differ in other spectrometers than the Perkin-Elmer Lambda-9. As opposed to a plain optical cuvette, a cavity changes the imaging properties of the spectrometer when placed into the sample volume. This alteration can be more or less severe and has to be taken into account. 
( $\ell \sim 80 \mathrm{~mm}$ ) was smaller than the radius of curvature of the mirrors $|r|=200 \mathrm{~mm}$, which is not critical as long as the cavity remains optically stable. In the Lambda9 spectrometer the beam entering and leaving the sample chamber (start and tip of arrows in Fig. 4.2 has a diameter of $\approx 10 \mathrm{~mm}$, the focus is in the middle of the sample chamber. The reflectivities, $R_{1}$ and $R_{2}$, of the cavity mirrors were equal in this $\operatorname{setup}\left(R=R_{1} \approx R_{2} \approx 0.99\right.$, from $\lambda=415$ to $\left.640 \mathrm{~nm}\right)$. The transmitted intensity of the beam was strongly reduced by the mirrors by approximately $\left(1-\sqrt{R_{1} \cdot R_{2}}\right) / 2$ (cf. Eq. 2.4), even without the sample in the cavity. Therefore the analog-to-digital capability of the spectrometer is not fully exploited considering the strong signal in the reference beam. A third mirror (identical with the other two mirrors forming the cavity, $\left.R_{3} \approx 0.99\right)$ was thus placed in the reference beam, (see Fig. 4.2) reducing the transmitted intensity by $\left(1-R_{3}\right)$. In that way the transmitted intensities of the two beams differed only by about a factor of 0.5 , enabling almost the full scale of the A/D converter to be used. The following spectrometer settings were used: scan speed $60 \mathrm{~nm} / \mathrm{min}$, slit width corresponding to $1 \mathrm{~nm}$ band-width, averaging time constant $0.5 \mathrm{~s}$. For comparison single pass experiments without the optical cavity in place were performed. In these experiments the spectrometer was run in the conventional manner using the same settings. Only the cuvette was placed in the sample beam, the reference beam was left blank, since the four air-glass surfaces of a cuvette would have caused additional unnecessary losses.

A home-made apparatus employing a high power short-arc Xe lamp was also used to evaluate the limits of IBBCEAS on basis of the benzene overtone spectrum. The setup is shown schematically in Fig. 4.3. The light from the arclamp was focused through a cutoff-filter $(560 \mathrm{~nm})$ into an optical cavity (the same

The requirements concerning an optimization of the imaging of the cavities multi-mode response with respect to the spectrometers detection system varies from setup to setup and cannot easily be generalized. 


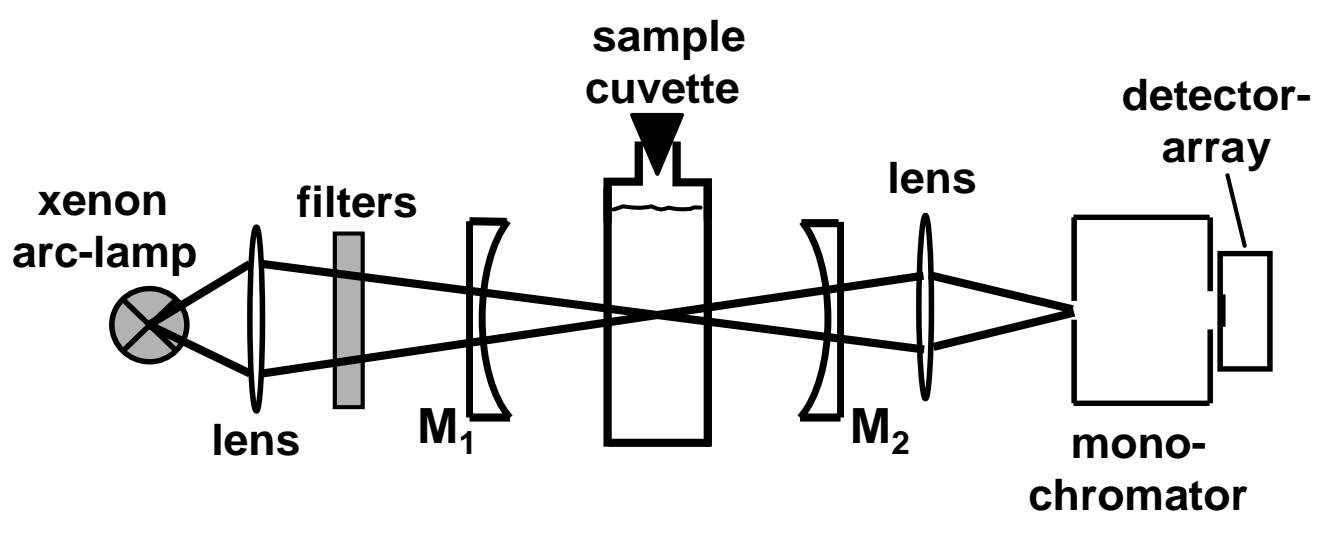

Figure 4.3: Schematic of a home-built IBBCEAS setup employing a high luminosity

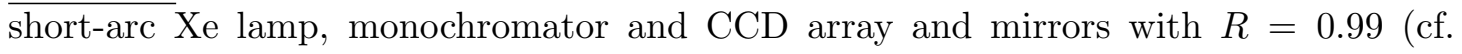
Fig. 4.4).

mirrors were used as above, $R \approx 0.99$ ) containing a cuvette with a $1 \mathrm{~mm}$ path length. The transmitted light was focused to the slit $(100 \mu \mathrm{m})$ of a spectrometer (600 lines/mm grating, focal length $250 \mathrm{~mm}$ ). A CCD-array (Sony ILX511) at the position of the exit slit measured the broad-band transmitted intensity spectrum with a resolution of $0.9 \mathrm{~nm}$. Owing to the high luminosity of the lamp (specified as $18 \mathrm{~W} \mathrm{~cm}^{-2} \mathrm{sr}^{-1} \mathrm{~nm}^{-1}$ at $400 \mathrm{~nm}$, ISAS Berlin) a neutral density filter in front of the cavity had to be used even at the shortest possible exposure time of the CCD array $(1 \mathrm{~ms})$, in order to reduce the intensity by a factor of $\approx 10$. The analog voltage signal of the CCD was measured with an 11-bit vertical resolution of a digital oscilloscope (LeCroy 9450A) by averaging over 5000 samples. For a detailed description see section 3.1 .

\subsection{Results}

The results of IBBCEA and single pass absorption measurements for three benzenefilled cuvettes of different path length (i.e. $d=0.1,1$, and $10 \mathrm{~cm}$ ) are shown in Fig. 4.4 between 450 and $650 \mathrm{~nm}$. The absorption values of the single pass ex- 


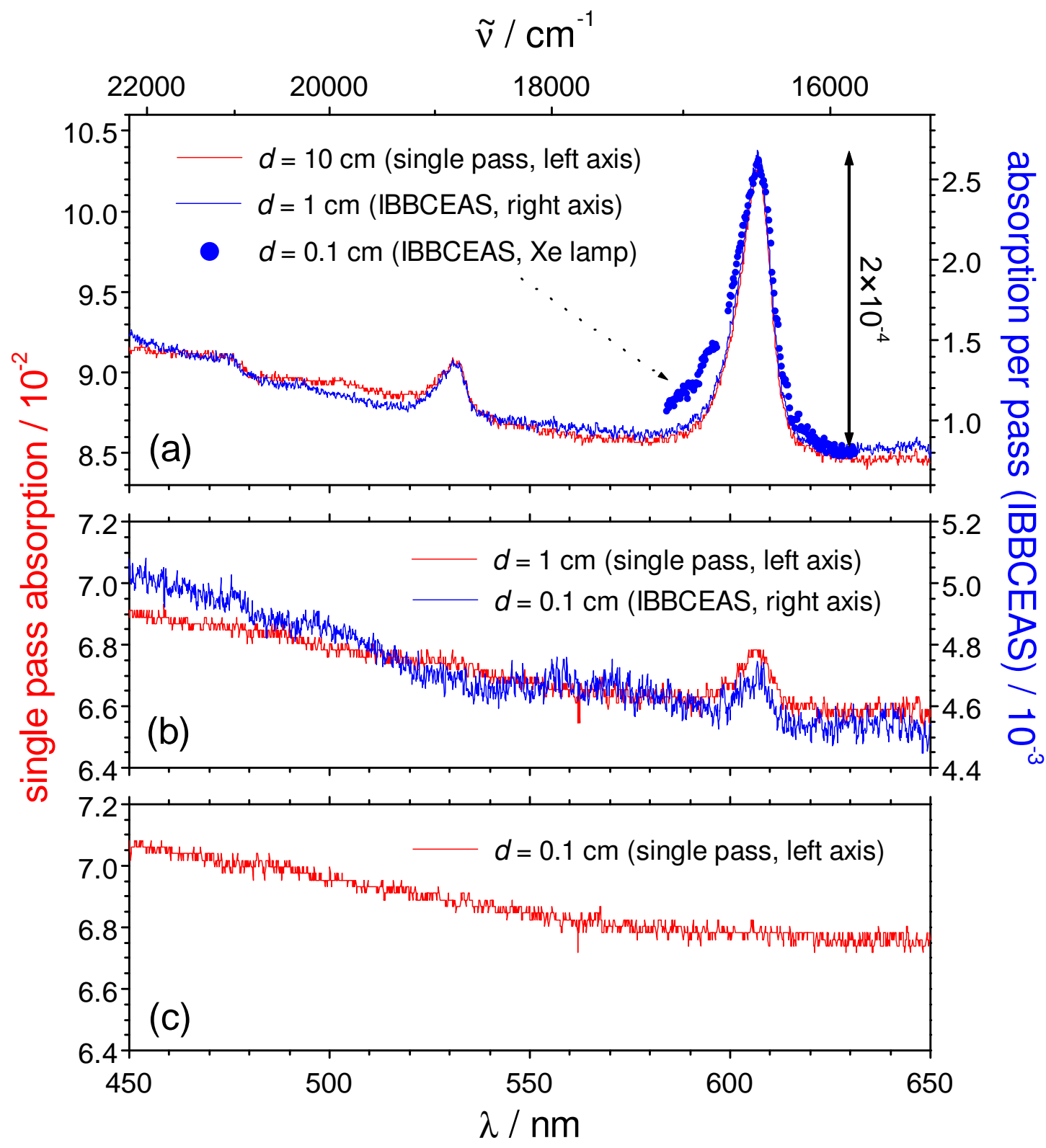

Figure 4.4: Comparison of single pass (left axis) and IBBCEA (right axis) absorption measurements of pure liquid benzene in cuvettes with different path lengths, $d$, given in the figure. The scaling on the left axis is ten times larger than on the right axis with slightly different offsets but the same distances from tick to tick. All spectra were measured with a standard Perkin-Elmer Lambda-9 double-beam spectrometer (Fig. 4.2) except for the one in a) represented by the solid blue circles ( $\bullet$ ") which was measured with the home-built setup shown in Fig. 4.3. This spectrum is normalized to the measurements taken with the Lambda-9 for comparison (axis not shown - absorption per pass $\approx 2 \times 10^{-4}$ at $\lambda=606.7 \mathrm{~nm}$ (maximum) indicated by vertical double arrow). 
periments are larger due to the different impact that back reflection of light from the cuvette windows has on the measurement. In the IBBCEA measurement back reflection losses are much less severe, since the reflected light is not lost inside the cavity, whereas in the single pass experiment the reflection losses significantly contribute to the overall absorbance. For all measurements the reflection losses of the cell windows $(\sim 7 \%)$ were not subtracted. Spectra with a similar signal-to-noise ratio are summarized in Fig. 4.4. Note that the scaling on the left axis is ten times larger than on the right axis with slightly different offsets but the same distances from tick to tick. Thus the relative division on the vertical axes differs exactly by a factor of 10 .

While in the single pass experiment for a cuvette with $0.1 \mathrm{~cm}$ path length no absorption feature can be seen at all (Fig. 4.4c), in the IBBCEA experiment using the same cuvette the fifth $\mathrm{C}-\mathrm{H}$ stretch overtone of benzene at $607 \mathrm{~nm}$ is already clearly visible (Fig. 4.4b). This IBBCEA spectrum compares well with a single pass experiment taken with a cuvette of $1 \mathrm{~cm}$ path length. The spectra taken with cuvettes of $1 \mathrm{~cm}$ (IBBCEAS) and $10 \mathrm{~cm}$ (single pass) path lengths in Fig. 4.4a also compare rather well. Fig. 4.4 also shows clear evidence of the sixth C-H stretching overtone of benzene at $531 \mathrm{~nm}$ and even a feature that can be attributed to the seventh overtone at $475 \mathrm{~nm}$ Reddy82. Since the noise of the light intensity for the single pass measurements was close to the analog-to-digital converting limit, the signal-to-noise ratio was even improved by a factor of $\sim 10$, which in fact is larger than the expected factor of 7 for mirrors with $R=0.99$ (see section 2.3.3). Better mirrors $(R \approx 0.9998)$ could still improve the enhancement of the sensitivity from an expected factor of 7 to 27 (see Fig. 2.4), because the additional losses by the cuvette windows are merely of the order of $10^{-3}$ per pass.

The circular symbols ("•") in Fig. 4.4 a were measured with the home-built setup in Fig. 4.3 using the same mirrors $(R=0.99)$ as for the other measurements shown 
in Fig. 4.4. The high luminosity of the Xe-arc lamp reduces the quantum noise strongly and therefore a much larger signal-to-noise ratio was achieved, even for a cuvette with $d=0.1 \mathrm{~cm}$. The absorption per pass for this spectrum (no absorption axis shown - see vertical arrow in Fig. 4.4 a) is approximately 10 times smaller than indicated on the right axis of Fig. 4.4 a. Even though the total exposure time in this experiment was only $5 \mathrm{~s}$, the slow 11-bit A/D conversion caused the overall measurement duration to be $\approx 500 \mathrm{~s}$.

\subsection{Discussion}

CRDS on benzene in the liquid phase was first demonstrated by Xu et al. [Xu02, who described measurements of different benzene concentrations in hexane. The path length of their Brewster window sample cell was $1 \mathrm{~cm}$ inside an optical cavity (length $48 \mathrm{~cm}$ ) formed by mirrors with a reflectivity of $R=0.9997$. The reported absorption per pass of $\alpha=14 \times 10^{-4} \mathrm{~cm}^{-1}$ at the band maximum at $607.1 \mathrm{~nm}$ $\left(\approx 16471 \mathrm{~cm}^{-1}\right)$ can be compared with our results of $\alpha=(17.2 \pm 0.4) \times 10^{-4} \mathrm{~cm}^{-1}$ at $\lambda_{\max }=606.9 \pm 0.3 \mathrm{~nm}$ for both the single pass and the IBBCEAS experiments. Although the absorption maximum differs slightly, other groups have given different values between $6 \times 10^{-4} \mathrm{~cm}^{-1}$ and $22 \times 10^{-4} \mathrm{~cm}^{-1}$ for the amplitude of this absorption band [Swofford76, Stone78, Patel79]. The asymmetric form of the band is in good agreement with CRD measurements presented by $\mathrm{Xu}$ and co-workers. Their data show that due to different refractive indices of the components of binary mixtures (benzene and hexane) inevitably occurring striae significantly increase the noise of the absorption spectra in comparison to normal one-component liquids. Based on Fig. 2 in Ref. $\mathrm{Xu02}$ their noise of the measured absorption coefficient was estimated to be of the order of $\sim 4 \times 10^{-5} \mathrm{~cm}^{-1}$ for the pure benzene sample. This value can be compared with the standard deviation of the absorption noise 
of $\approx 2 \times 10^{-5}$ per pass for the IBBCEAS measurements using a cuvette with $1 \mathrm{~cm}$ path length in the Lambda-9 spectrometer. Despite the low mirror reflectivities $(R \approx 0.99)$, the noise in the IBBCEAS experiment is even slightly better in comparison to the CRD measurement in [Xu02]. For the IBBCEA setup employing the Xe-arc lamp the standard deviation of the noise is $\sim 3 \times 10^{-6}$ per pass, however, the path length in this measurement was only $0.1 \mathrm{~cm}$. The IBBCEAS detection limit with a bright light source is hence approximately $6 \times 10^{-6}$ (twice the noise) per pass for mirrors with only $R \approx 0.99$.

The absorption of the fifth $\mathrm{C}-\mathrm{H}$ stretch overtone of benzene has also been studied in the gas-phase using pulsed CRDS in a cavity of $142.8 \mathrm{~cm}$ length and a mirror reflectivity of $R=0.9993$ [Kleine99. The absorption coefficient in the maximum of the absorption peak was of the order of $1.2 \times 10^{-6} \mathrm{~cm}^{-1}$ at $16549.8 \mathrm{~cm}^{-1}$ $(\approx 604 \mathrm{~nm})$ and the FWHM was $\sim 96 \mathrm{~cm}^{-1}(\sim 3.5 \mathrm{~nm})$, in agreement with other data presented by Reddy et al. Reddy82, who studied the $\mathrm{CH}$ and $\mathrm{CD}$ stretch overtone excitations of gas phase benzene with a dye-laser photo-acoustic method. In comparison, the empirical FWHM of the $6 \nu_{C} H$ absorption band in Fig. $4.4 \mathrm{a}$ is $\approx 250 \mathrm{~cm}^{-1}$, due to strong inhomogeneous broadening in solution.

\subsection{Summary and Outlook}

Incoherent broad-band cavity-enhanced absorption spectroscopy (IBBCEAS) combines the simplicity and robustness of standard non-invasive optical absorption spectroscopy with the enhancement concepts developed in cavity ring-down spectroscopy. In this section it was demonstrated how IBBCEAS can be applied to liquid samples in a straightforward manner using either a standard commercial absorption spectrometer or a simple home-built setup. 
The presented IBBCEAS experiments of liquid benzene (as well as not presented experiments using acetonitrile and methanol in the same setup) confirmed the expected signal-to-noise ratio enhancement (section 2.3.3) compared to single pass measurements even for relatively high additional losses by cuvette windows. In practice the cuvette windows could be turned into highly reflecting mirrors by coating them from the outside with an appropriate dielectric material. The light would then be transmitted by only two instead of four surfaces, which reduces the losses. Preferably plano-convex windows should be used in order to ensure the cavity to stay within the limits of optically stable resonator geometries. 


\section{Chapter 5}

\section{Naphthalene Gas Phase Oscillations}

Oscillations and dynamic instabilities occur in a variety of chemical systems. However, the majority of such "exotic phenomena" are observed either in the condensed phase [Epstein98, Field85], in gas flow reactors or combustion reactions [Scott90, Scott94]. The serendipitous discovery of a new closed gas phase system was reported by Ruth et al. Ruth02b] exhibiting unusual periodic and complex dynamical behaviour. The original objective was to test whether UV multi-photon ionization (MPI) is a viable way to selectively create a sufficient concentration of polycyclic aromatic hydrocarbon (PAH) cations in a supersonic expansion, for absorption measurements of long wavelength $\pi-\pi$ transitions, using the sensitive cavity ring-down (CRD) technique ['Keefe88, Wheeler98. The prime motivation was to find a method that could replace the already established, but much less selective, electrical discharge approach for the production of ionized carbon chains Motylewski99 and/or cationic PAHs [Romanini99]. Our long term ambition was to study the absorption of cationic PAHs in a supersonic jet. Therefore gaseous 
naphthalene in a static cell buffered by a noble gas as a first "well known" test case for preliminary MPI experiments.

The absorption of the MPI products of naphthalene was at first measured at $\approx 648.84 \mathrm{~nm}$, a wavelength where a strong transition ${ }^{2} B_{3 g}\left(D_{2}\right) \leftarrow X^{2} A_{u}\left(D_{0}\right)$ of gaseous cationic naphthalene is expected [Romanini99, Pino99] and neutral naphthalene is completely transparent. It turned out that the time-dependence of the absorption, measured after UV multi-photon excitation of the gas mixture, is rather extraordinary and non-trivial. Depending on the experimental conditions, the absorption of the multi-photon photolysis products exhibited different types of temporal behaviour, ranging from simple growth/decay patterns, via oscillatory responses to complex dynamics, all on a timescale of seconds to many minutes. These unexpected observations made us revise our original objectives and led to the investigation of this new nonlinear gas phase phenomenon [Ruth02b]. A detailed description of these experiments varying different parameters of the setup and using CRDS to detect the absorption is given in Gash04]. Nonetheless with the existing data no evidence of the carriers of the oscillating absorption was found since absorption spectra could not be measured. Due to the time depending absorption signal a wavelength scan of the laser was inappropriate to gather spectral information.

Because of its broad-band capabilities and fast data acquisition, the IBBCEA method was the first choice to measure the time depending absorption spectra of these oscillations. To explain the oscillation phenomenon to the reader, the CRD setup used first and a short overview of the CRD results is given below (refer also [Ruth02b] and Gash04]), followed then by the presentation and discussion of new experimental data measured with IBBCEAS. 


\subsection{CRDS - Setup}

The experimental setup is shown schematically in Fig. 5.1. It consists of a wellpumped static vacuum cella which was filled with gaseous naphthalene at its room temperature vapour pressure $\left(\mathrm{P}_{\mathrm{Nap}} \sim 0.1 \mathrm{mbar}\right)$ vanderLinde98 and buffered with either helium or argon. The stagnation pressures $\mathrm{P}_{\mathrm{He}}$ for helium and $\mathrm{P}_{\mathrm{Ar}}$ for argon were typically between $\sim 5$ and 135 mbar and between $\sim 5$ and 30 mbar, respectively. Naphthalene was excited in a resonance enhanced $n$-photon absorption process $(\mathrm{n} \geq 1)$ using the UV output from a pulsed excimer laser at $308 \mathrm{~nm}$ (Lumonics EX-700, XeCl, $\sim 15 \mathrm{~ns}$ duration, $\mathrm{E}_{\text {pulse }}$ typically between 5 and $35 \mathrm{~mJ}$, measured by a Gentec ED-500L joule meter (JM) after being transmitted through the quartz windows of the chamber). The excitation beam was focused with a lens into the static gas chamber onto the optical axis of a high finesse cavity. The excitation conditions are estimated to be well below the breakdown threshold of Ar or He in comparison with literature data [Turcu97, Sircar96]. In typical experiments the gas mixture was exposed to $\mathrm{N}_{\text {pulse }}$ laser pulses at a repetition rate of either 10 or $20 \mathrm{~Hz}$, with $\mathrm{N}_{\text {pulse }}$ typically being between 10 and 100 . Immediately after blocking the UV laser beam with a shutter, the absorption of the photoproducts formed in this multi-photon photolysis process was measured as a function of time at a selected fixed wavelength between 635 and $665 \mathrm{~nm}$ (Lumonics HD-300 dye laser, rhodamine 101) using the cavity ring-down (CRD) technique. In this wavelength region neutral gaseous naphthalene is completely transparent. An Angstrom WS7 wavemeter (WLM) measured the wavelength of the dye laser. The cavity used for the absorption measurement in the experiment was formed by two highly reflecting $(\mathrm{HR})$ spherical mirrors $(R \approx 0.9998)$ with a separation of $66 \mathrm{~cm}$. The transmitted intensity was measured by an "EMI 9558QB" photomultiplier tube

a The vacuum chamber is a stainless steel six-way cross piece with an approximate volume of $\mathrm{V} \approx 5 \mathrm{dm}^{3}$. 


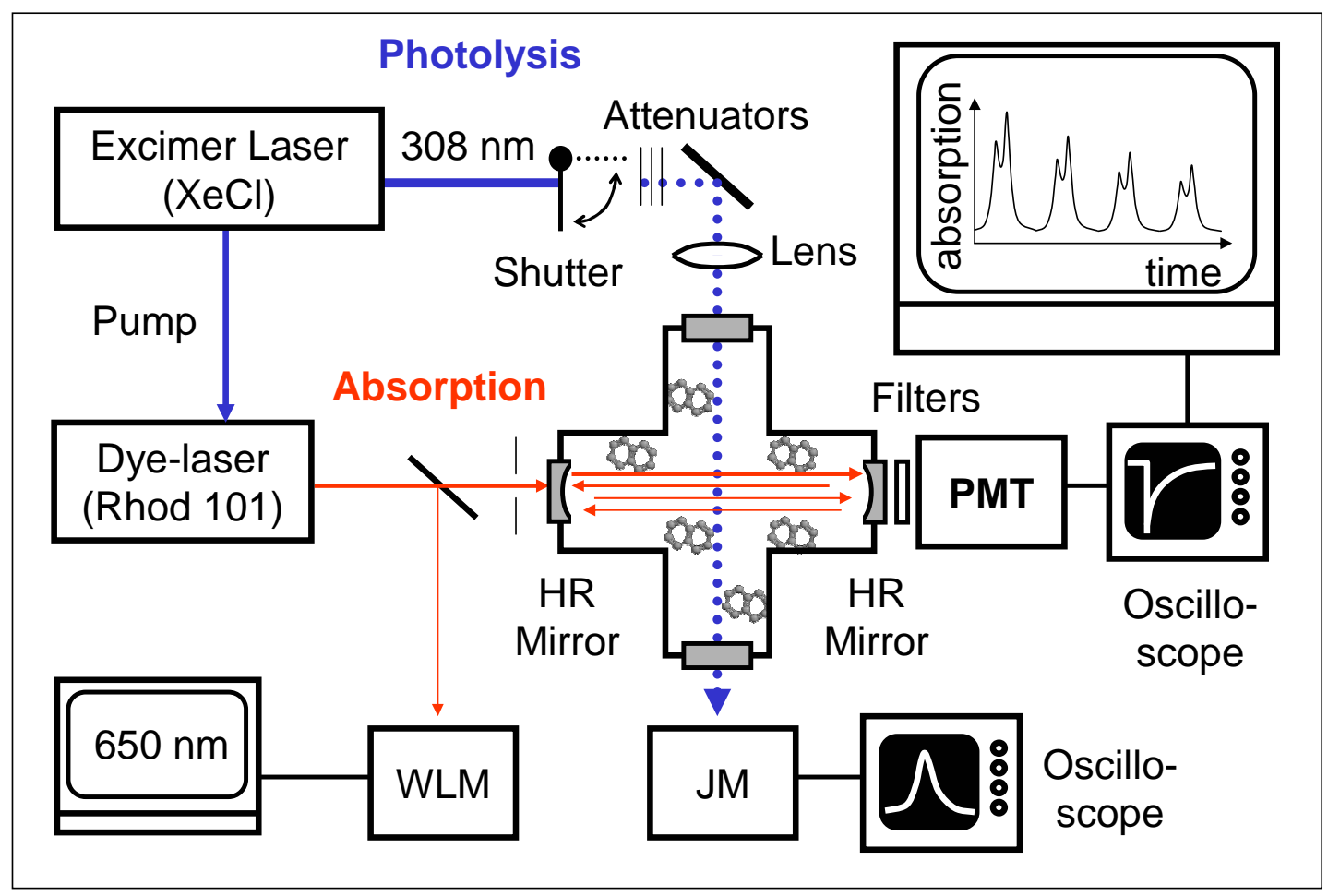

Figure 5.1: Schematic illustration of the experimental setup [Gash04]. For the photolysis of naphthalene the UV beam is focused into the centre of the static gas cell. The shutter blocks the UV beam during the cavity ring-down absorption measurement. (WLM: Wavemeter, PMT: Photomultiplier tube, JM: Joule meter).

(PMT) and a digital oscilloscope (Lecroy 9410) which averaged several ring-down decays. Typical cavity ring-down times, $\tau_{\text {crd }}$, in the non-absorbing gas mixture before photolysis were $\sim 14 \mu \mathrm{s}$.

For conditions, as they are outlined above, the UV multiphoton photolysis products of the naphthalene gas mixture exhibited absorption properties over the entire wavelength region considered (635 to $665 \mathrm{~nm}$ ). Within this wavelength region the absorption was found to be strongly time-dependent, revealing unusual dynamical features. Consequently measurements were only taken at certain fixed wavelengths. 


\subsection{CRDS - Results and Classification of Oscillation Types}

The time-dependence of the absorption is extremely sensitive to the specific experimental conditions; the most critical parameters turned out to be (i) the partial pressure of the buffer gas and of naphthalene and (ii) the number and energy of the UV pulses used in the multi-photon photolysis, implying different photon fluences.

Depending on the type of buffer gas and its partial pressure three cases of dynamical behaviours of the absorption can be distinguished:

Type I: Simple growth and subsequent decay. At "low" partial pressures of the buffer gas a fast rise in the absorption is followed by a monoexponential decay of the absorption with lifetimes between $\sim 2$ and $\sim 23 \mathrm{~s}$. The term low partial

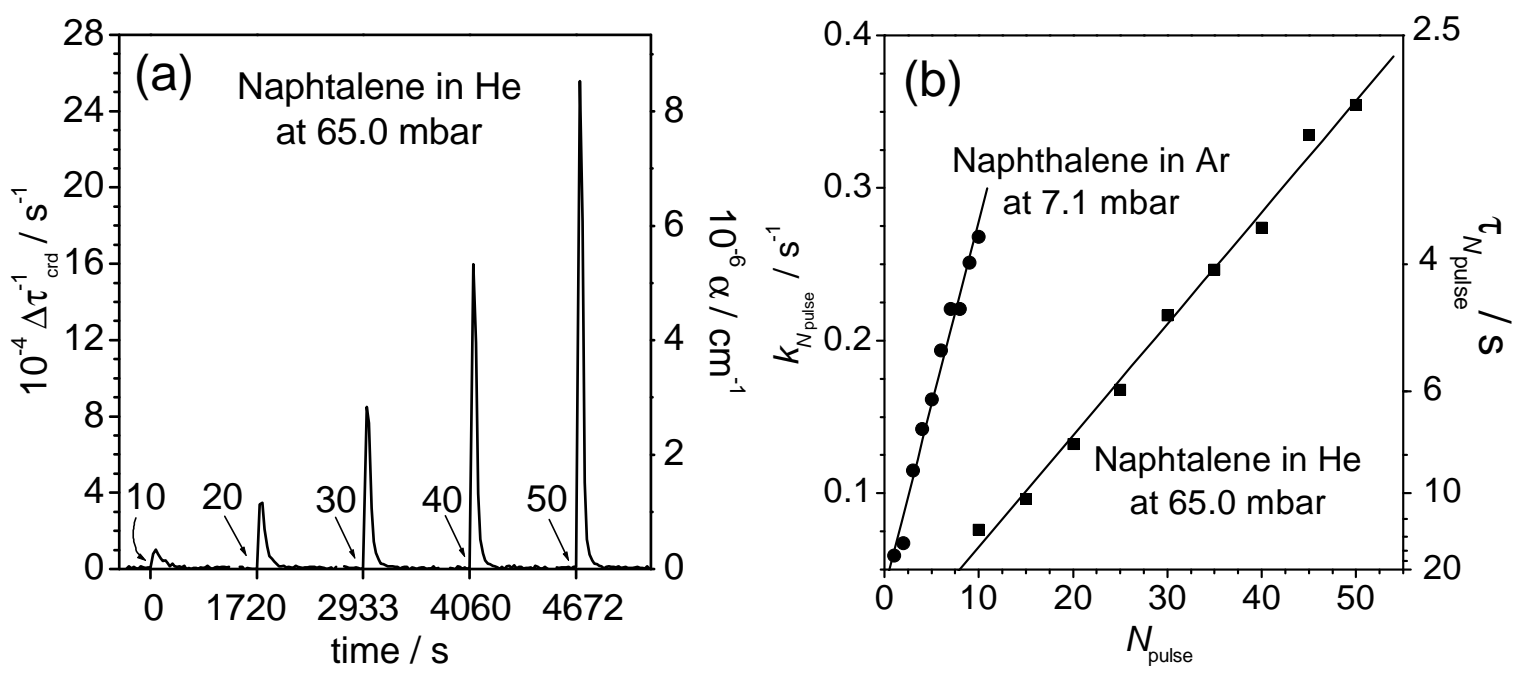

Figure 5.2: Example for Type I behaviour: Simple growth and subsequent decay. (a) Absorption coefficient $\alpha$ of the resonance-enhanced multiphoton photolysis products of gaseous naphthalene in He measured at $650 \mathrm{~nm}$ after excitation with 10, 20, 30, 40 and $50 \mathrm{UV}$ laser pulses at $20 \mathrm{~Hz}$ (arrows indicate excitation times). The decay follows a single exponential function with rate coefficient $k_{\mathrm{N}_{\text {pulse }}}$. The maximum absorption depends on the number of pulses, $N_{\text {pulse }}$. (b) $k_{\mathrm{N}_{\text {pulse }}}$ as a function of $N_{\text {pulse }}$ for measurements in buffer gases He and Ar. 
pressure refers to the range of $\sim 5$ to $\sim 70$ mbar in He and $\sim 5$ to $\sim 7.5$ mbar in Ar. This type of temporal response of the system is most reproducible; the reproducibility is subject to the degradation of the initial gas mixture. An example of growth and subsequent decay at low pressures for different numbers of UV shots is shown in Fig. 5.2(a). The rate coefficient of the monoexponential decay $\mathrm{k}_{\mathrm{N}_{\text {pulse }}}$ was found to increase linearly with increasing number of UV photolysis pulses as shown in Fig. $5.2(\mathrm{~b})$.

Type II: Quasi-periodic oscillations with one or more components. At "medium" partial pressures of the buffer gas oscillations in the strong absorption of the photolysis products are observed with amazingly long periods ranging from seconds up to several minutes. The total oscillatory behaviour can persist for several hours (see Fig. 5.3 . The term medium partial pressure refers to the range

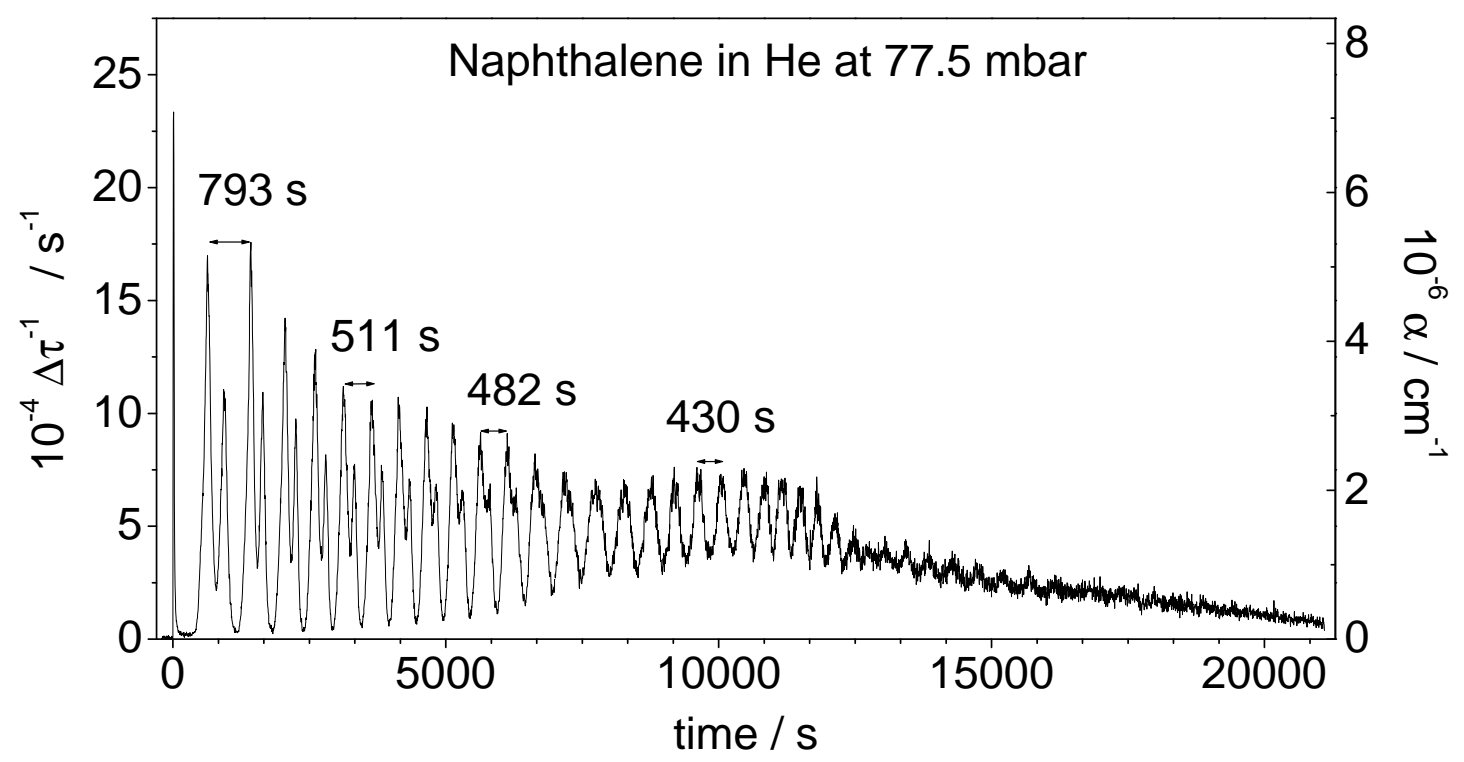

Figure 5.3: Example for Type II behaviour: A Quasi-periodic oscillations of the absorption coefficient with two components, measured in He at $650 \mathrm{~nm}$ after excitation (at $t=0)$ with $20 \mathrm{UV}$ laser pulses at $20 \mathrm{~Hz} ; P_{\mathrm{Nap}}=0.10 \pm 0.01 \mathrm{mbar}$. The change of the period is indicated for one of the components; $E_{\text {pulse }}=26.2 \pm 2.2 \mathrm{~mJ}, P_{\mathrm{He}}=77.5 \pm 0.2 \mathrm{mbar}$, $T=24.1 \pm 0.2^{\circ} \mathrm{C}$. For similar conditions (changes of less then $2 \%$ ) significantly different oscillations of shorter periods (by a factor of more than 10) can be observed. 
of $\sim 70$ to $\sim 80$ mbar in He and $\sim 7.5$ to $\sim 12$ mbar in Ar. The oscillatory time dependence, Fig. 5.3, is typical of numerous measurements. Virtually all oscillations obtained are only reproducible qualitatively, i.e. the periods and amplitudes can vary significantly from measurement to measurement. The absorption during oscillations can be an order of magnitude stronger than in simple growth/decay responses described for Type I; amplitudes can be as large as $\Delta \alpha \sim 3 \times 10^{-5} \mathrm{~cm}^{-1}$. The oscillatory response often consists of more than one independently varying component (see Fig. 5.3). The character of the oscillations is extremely dependent on all relevant experimental parameters. Some of the temporal structure could be empirically described by Gaussian profiles.

Type III: Apparently complex oscillatory behaviour. At "high" partial pressures of the buffer gas, and on certain occasions also at pressures which were

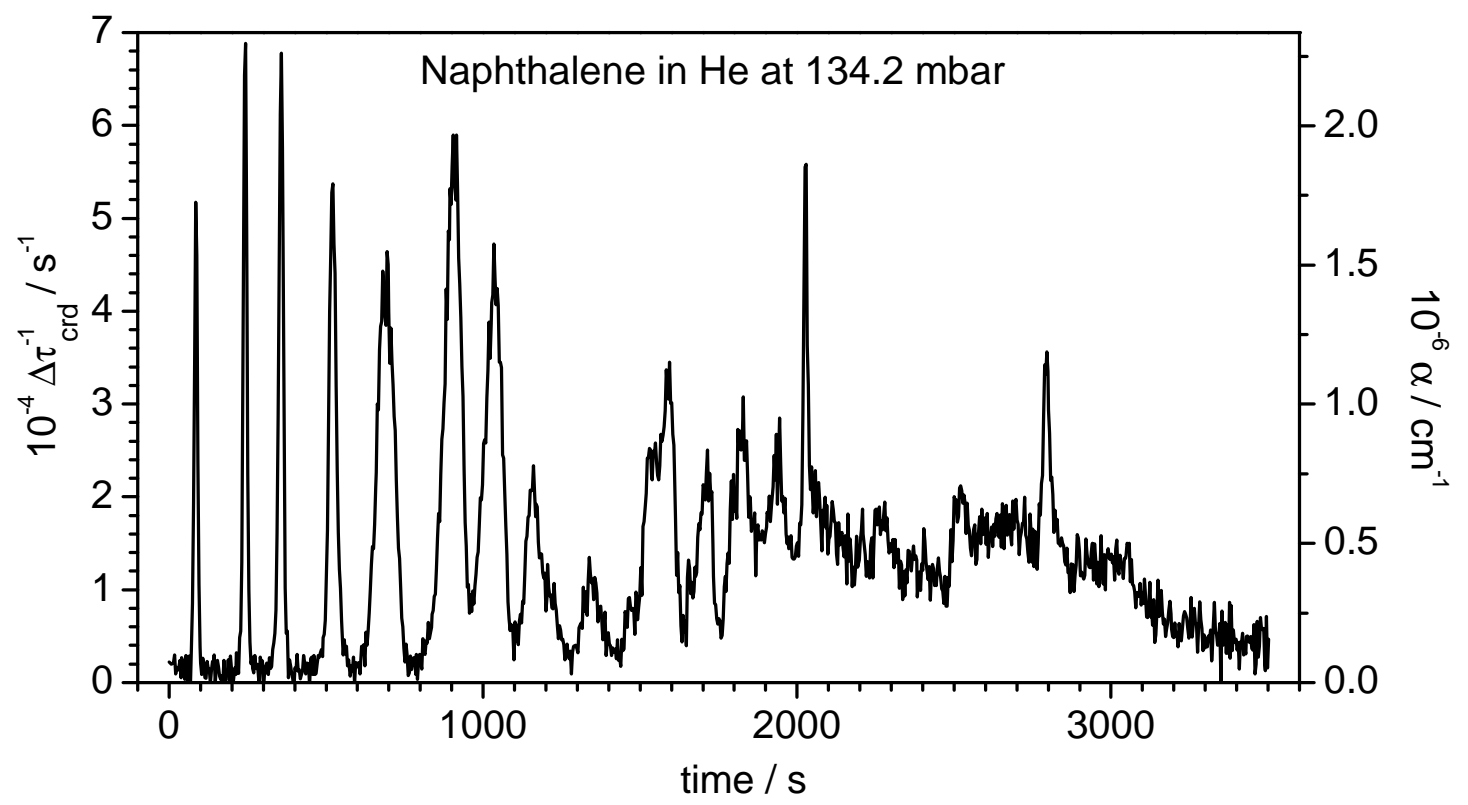

Figure 5.4: Example for Type III behaviour: Apparently complex oscillatory behaviour of the system, which develops at high buffer gas pressure. Measurement of gaseous naphthalene in He measured at $650 \mathrm{~nm}$ after excitation (at $t=0$ ) with $10 \mathrm{UV}$ laser pulses at $10 \mathrm{~Hz}$; parameters: $P_{\mathrm{Nap}}=0.09 \pm 0.01 \mathrm{mbar}, P_{\mathrm{He}}=134.2 \pm 0.2 \mathrm{mbar}, E_{\text {pulse }}=$ $10.8 \pm 1.3 \mathrm{~mJ}, T=23.1 \pm 0.2^{\circ} \mathrm{C}$. 
categorized as "medium", complex temporal responses, as shown in Fig. 5.4, were observed. Since periodic oscillations and complex temporal responses (Type II and Type III) are extremely dependent on all relevant experimental parameters, oscillations as shown in Fig. 5.4 are virtually impossible to reproduce other than qualitatively. That way it is tempting to attribute chaotic properties to the system, however, there is no evidence for such an assignment yet.

\subsection{CRDS - Discussion}

Additionally, it was established that for conditions when the system was continuously exposed to UV pulses during the CRD interrogation (i.e. the shutter in Fig. 5.1 is left open) a slow but continuous increase of the absorption was observed, followed by strong oscillatory behaviour only when the UV light was cut off $b$. This important fact shows that the UV radiation prevents the formation of one or even several components, which are essential for the occurrence of oscillations. Vestiges of molecular oxygen and nitrogen at levels of $<5 \times 10^{-3}$ mbar may be present in the static cell. Under several (important) experimental conditions no absorption was observed after multi-photon UV excitation!c

1. When the UV laser beam was not focused.

2. With only the buffer gas present in the cell but no naphthalene vapour.

3. With only naphthalene present in the cell (no buffer gas).

4. Under typical experimental conditions stirring of the gas mixture with a fan removes oscillatory behaviour, however using specific experimental conditions strongly damped oscillations were still observable.

b In fact, our first experiments were conducted that way, we subsequently changed to the procedure described in the experimental paragraph.

c The dependence of the dynamics on the gas chamber size has not yet been tested. 
The most striking feature of this gas phase system is the remarkably long time that it is able to remain away from an equilibrium state. The timescale on which it evolves seems unique for a gas phase experiment. No experimental results that can be directly compared with the presented data could be found in the literature. In Gash04 it was demonstrated that a nonlinear chemical reaction model using a pseudo-first order reaction scheme can yield results which qualitatively agree with the absorption behaviour of type I shown by this experiment. It considers two starting reactants $\mathrm{Q}$ and $\mathrm{S}$ which react over two intermediate steps $\mathrm{A}$ and $\mathrm{B}$ to $\mathrm{C}$. In appendix A.2 the model is explained in detail. If additionally an autocatalytic reaction step, $\mathrm{A}+2 \mathrm{~B} \rightarrow 3 \mathrm{~B}$, is introduced into the model, the reaction scheme becomes nonlinear. Depending on the reaction rates and on the initial concentrations of $\mathrm{Q}$ and $\mathrm{S}$ the concentration of reactant $\mathrm{B}$ shows a similar behaviour as was observed for the type I and II absorption in the experiments reported here. Several species were suggested to be B, the carrier of the observed absorption: Naphthalene excited to the metastable triplet state $T_{1}$, the naphthalene-isomer azulene, dissociation products $\left(\mathrm{H}, \mathrm{C}_{2} \mathrm{H}_{2}, \mathrm{C}_{2} \mathrm{H}_{4}, \mathrm{H}_{2}\right.$ or $\left.\mathrm{C}_{3} \mathrm{H}_{3}\right)$ as well as the associated ions and their chemical reaction products. Even the scattering of light at condensed reaction particles was proposed. Time-resolved spectra of the oscillating absorption are fundamental to rule out some of the possibilities considered in Gash04]. The IBBCEA technique has the qualities necessary to measure these spectra broad-band and fast enough . The setup is easy to modify since the same chamber and mirrors can be used. 


\section{$5.4 \quad$ IBBCEAS - Setup}

In this setup the CRD system to detect the absorption was replaced by the IBBCEA method. The light from a fibre optic cold light source (Schott KL,2500 LCD, $250 \mathrm{~W}$ halogen lamp) was focussed by a lens $(f=30 \mathrm{~cm})$ into the center of the ring down cavity (length $\ell=66 \mathrm{~cm}$ ). Different cut-off filters were used to limit the spectrum of the light to the high reflectivity region of the mirrors (Layertec, $R=0.9995$ at $662 \mathrm{~nm},|r|=200 \mathrm{~cm}, \varnothing 25 \mathrm{~mm})$. The reflectivity $R(\lambda)$ was measured for $636 \mathrm{~nm}<\lambda<688 \mathrm{~nm}$ by CRDS using a pulsed Lumonics HD-300 dye laser (rhodamine 101). A second lens $(f=15 \mathrm{~cm})$ focussed the light transmit-

\section{Photolysis}

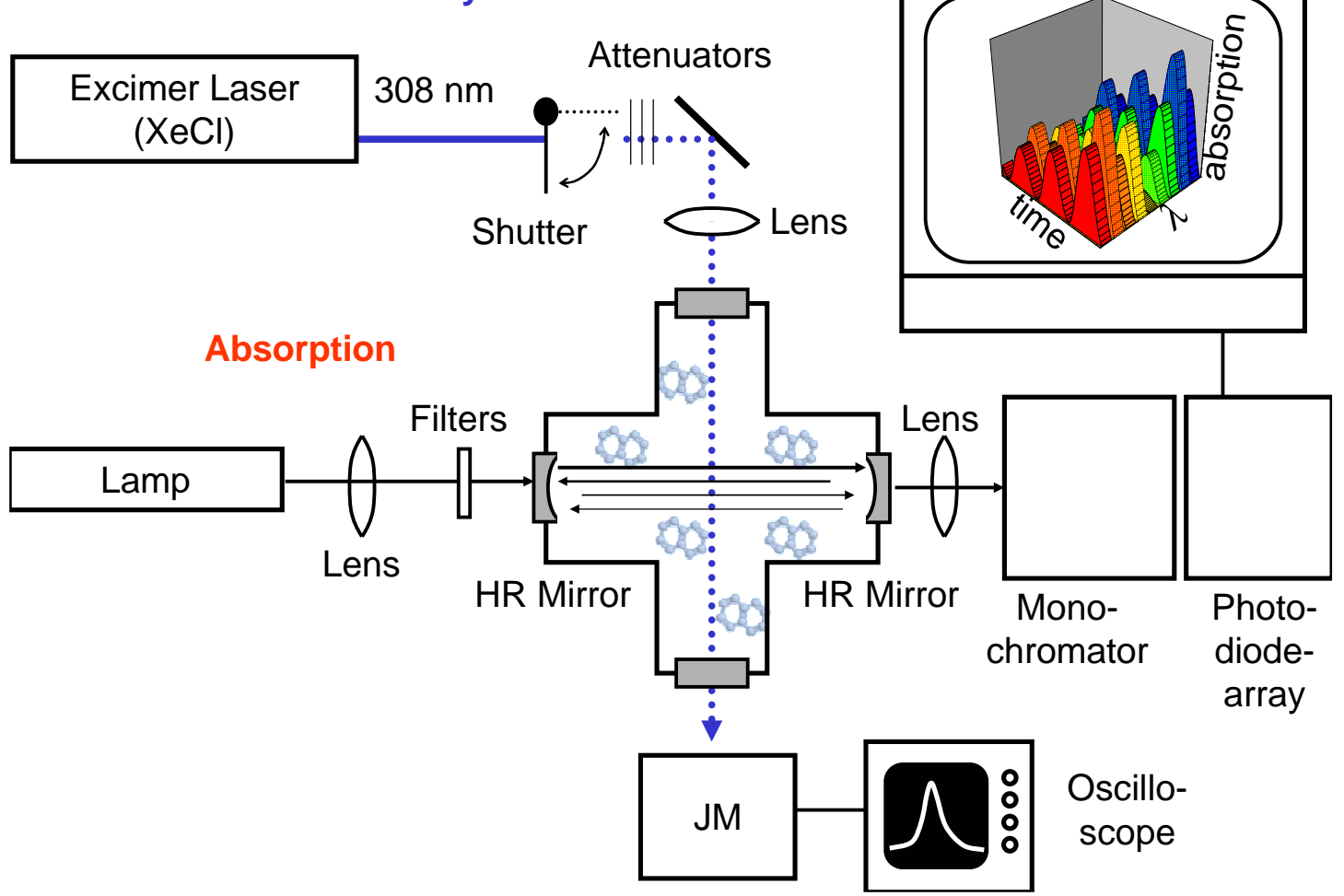

Figure 5.5: Schematic illustration of the IBBCEAS setup. For the photolysis of naphthalene the UV beam is focused into the centre of the static gas cell. The UV beam was not blocked by the shutter during the IBBCEA measurement. (JM: Joule meter). 
ted by the cavity onto the entrance slit (ca. $0.3 \mathrm{~mm}$ ) of a monochromator (B\&M Spektronik, 600 grooves/mm grating). The exit slit of the monochromator was replaced by a photodiode array (IRY-1024G, Princeton Instruments Inc., 1024 pixel at $25 \mathrm{~mm}$ ). The wavelength range of ca. $170 \mathrm{~nm}$ was calibrated by the light of a neon lamp and a spectral resolution $(F W H M)$ of $\approx 1.7 \mathrm{~nm}$ was achieved. The high reflectivity region of the mirrors limited the spectral region which could be investigated to $630-715 \mathrm{~nm}$. The minimum exposure time of the photodiodearray of $33 \mathrm{~ms}$ was used and 50 spectra were averaged. The time resolution for the IBBCEA measurements presented in this section is therefore $1.65 \mathrm{~s}$.

Naphthalene sublimated for more than eight hours into the evacuated chamber to ensure the thermodynamic equilibrium being established at room temperature. Then helium was filled in with a pressure of $p=80$ mbar. The temperature $T=22.0^{\circ} \mathrm{C}$ was measured in the vacuum chamber with a thermocouple. It drifted during the measurements by less than $\pm 0.4^{\circ} \mathrm{C}$. In the following figures concerning the IBBCEA measurements the absorption per pass is shown instead of the absorption coefficient $\alpha$ because the absorbing species is not necessarily homogeneously distributed over the path length of the cavity.

\subsection{IBBCEAS - The Absorption Spectrum as a Function of Time}

A time resolved IBBCEA spectra of the oscillating absorption is shown in Fig. 5.6. The absorption per pass $A$ is plotted versus the time and the wavelength $\lambda$. The profile of the time evolution is coloured in pink. The four narrow peaks in the first $650 \mathrm{~s}$ are due to four multi photon excitations of 20 shots with a pulse energy of approximately $45 \mathrm{~mJ}$. They follow a typical type I behaviour of simple growth 


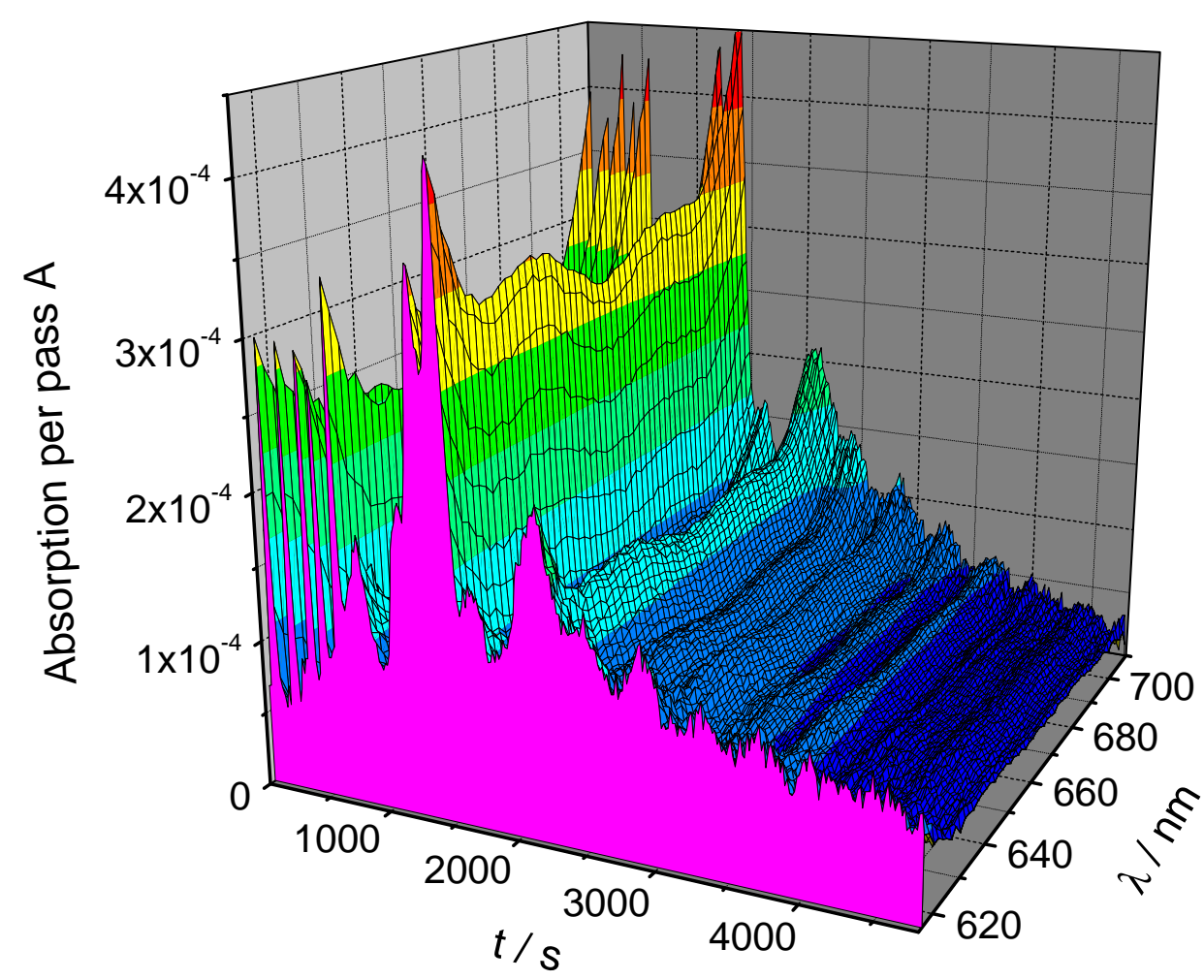

Figure 5.6: Time resolved IBBCEA spectra of the oscillating absorption after $4 \times 20$ shots of UV multi-photon laser $\left(E_{\text {pulse }}=45 \pm 2 \mathrm{~mJ}\right)$ photolysis of naphthalene; parameters: $P_{\text {Nap }}=0.10 \pm 0.01 \mathrm{mbar}, P_{\mathrm{He}}=80.0 \pm 0.2 \mathrm{mbar}, T=22.0 \pm 0.4^{\circ} \mathrm{C}$.

and subsequent decay. The oscillation triggered is of the type II and shows two superimposed periodic oscillations. The periods of approximately $160 \mathrm{~s}$ and $640 \mathrm{~s}$, respectively, increase with time while the amplitudes decay exponentially.

\subsection{IBBCEAS - Fit of the Spectrum}

The spectrum at the time of the highest absorption per pass, averaged over $28 \mathrm{~s}$, is shown in each graph of Fig. 5.7. With a standard deviation for the noise $\Delta A$ of $4 \times 10^{-7}$ the signal-to-noise ratio is of the order of 1000 . Considering a cavity length of $\ell=66 \mathrm{~cm}$ a signal-to-noise ratio of 2 corresponds to a sensitivity limit of 


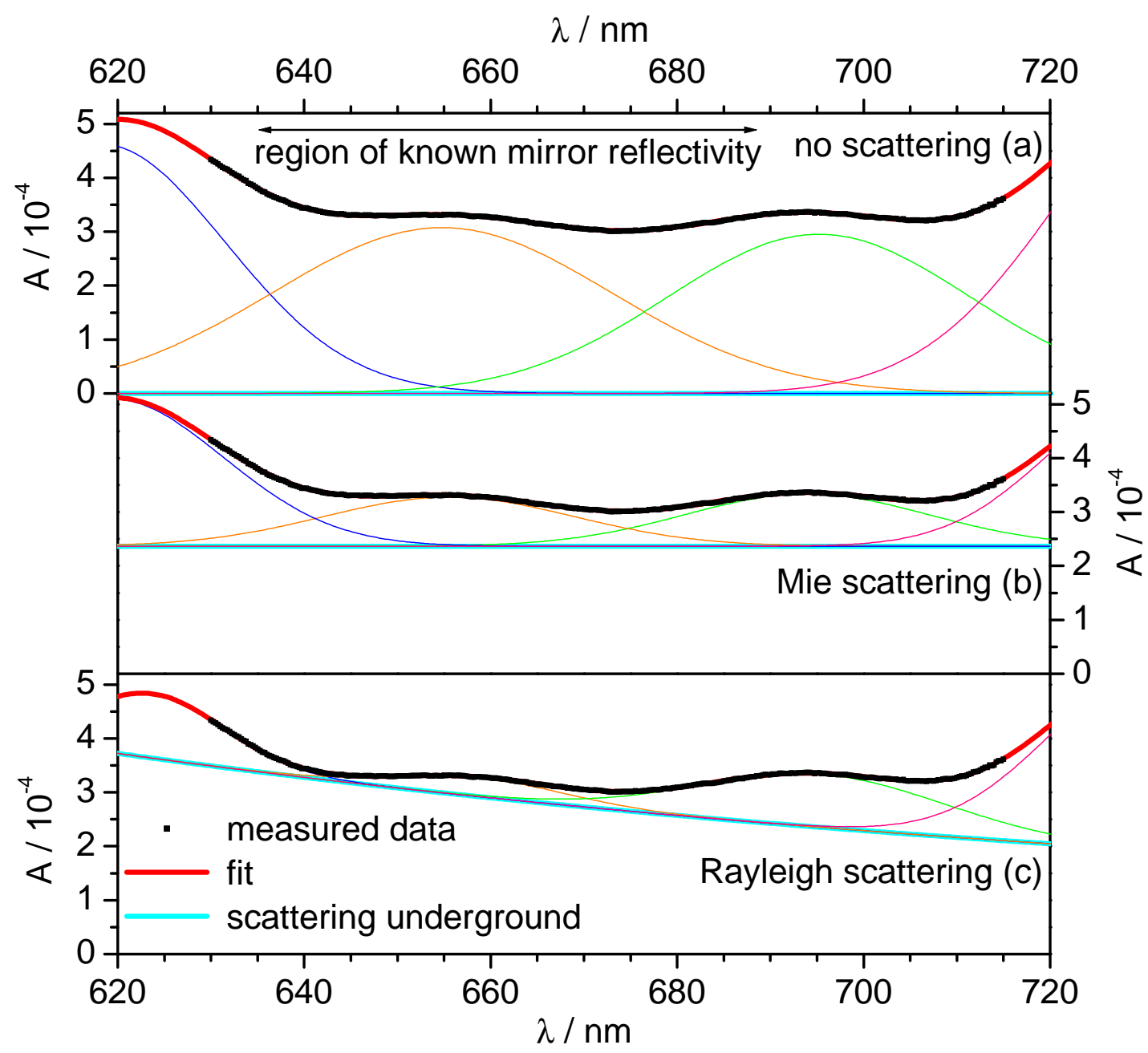

Figure 5.7: The measured absorption per pass A of the oscillation maximum is represented with black dots in all graphs. Different functions were fitted to the measured data considering four Gauss functions for the absorption features and (a) no scattering, (b) Mie scattering or (c) Rayleigh scattering background.

the absorption coefficient $\alpha$ of $1.2 \times 10^{-8} \mathrm{~cm}^{-1}$. Within these limits the measured absorption features of $\sim 30 \mathrm{~nm}$ width are smooth and without structure, despite the spectral resolution $(\mathrm{FWHM})$ of $\approx 1.7 \mathrm{~nm}$.

The fact that absorption features can be seen at all disproves the possibility discussed in Gash04], that only scattering from particles formed during photolysis, and not absorption, could be responsible for the decrease in the ring-down time 
measured following excitation. Nonetheless could scattering [Bohren83, Stover95] by particles or droplets, formed during or following the photolysis pulse, contribute in the observed oscillation of the optical loss. Precipitation of the naphthalene vapour, caused by the UV pulse, or another mechanism allowing for the creation of small particles may occur. It is possible for naphthalene following pyrolysis to undergo successive additions to form soot particles [Bockhorn94]. If these particles are suspended in the buffer gas, they will scatter the incident light.

If the average size of the particles is much smaller than the wavelength of the scattered light, Rayleigh scattering would be the dominant mechanism. The degree of scattering is then inversely proportional to the 4th power of the wavelength. According to the scattering theory of Mie [Born80] the wavelength dependence of the scattering cross section for particle sizes of the order of magnitude of the wavelength is strongly connected to the particle size. For the case that the particle sizes are larger than a few wavelengths the scattering loss is almost independent from the wavelength, herein after referred to as "Mie scattering".

The functions used to fit the measured absorption features considered besides four Gauss functions the different scattering mechanisms. The fits are shown in Fig. 5.7 where (a) no scattering background, (b) a constant wavelength independent Mie scattering background and (c) a Rayleigh scattering background $\left(\sim \lambda^{-4}\right)$ were assumed.

All functions used were suitable to fit the measured data. The fitted parameters of each absorption feature for the different scattering models are compared in Table 5.1. Since some of the fit parameters are correlated, the errors given by the fit procedure and added to the systematic errors are relatively high. The amplitude $A$ was normalized to the second feature $(\sim 655 \mathrm{~nm})$ and is given in arbitrary units (a.u.). The wavelength positions $\lambda$ of the absorption features differ for all fitted functions only within the error margins. Contrary to that the full 
Table 5.1: Parameters used to fit the measured IBBCEA spectrum for no scattering, Mie scattering and Rayleigh scattering background respectively.

\begin{tabular}{c|c|cccc} 
background & parameter & feature 1 & feature 2 & feature 3 & feature 4 \\
\hline \multirow{3}{*}{ (a) none } & A/a.u. & $1.5 \pm 0.4$ & $1.0 \pm 0.2$ & $1.0 \pm 0.2$ & $1.9 \pm 0.8$ \\
& $\lambda / \mathrm{nm}$ & $618 \pm 3$ & $655 \pm 2$ & $695 \pm 2$ & $736 \pm 8$ \\
& $w_{\text {FWHм }} / \mathrm{nm}$ & $32 \pm 4$ & $43 \pm 6$ & $38 \pm 5$ & $35 \pm 13$ \\
\hline \multirow{3}{*}{ (b) Mie } & A/a.u. & $2.6 \pm 0.7$ & $1.0 \pm 0.2$ & $1.0 \pm 0.2$ & $2.2 \pm 0.8$ \\
$\left(\sim \lambda^{0}\right)$ & $\lambda / \mathrm{nm}$ & $619 \pm 5$ & $655 \pm 2$ & $694 \pm 2$ & $730 \pm 8$ \\
& $w_{\text {FWHм }} / \mathrm{nm}$ & $28 \pm 8$ & $32 \pm 2$ & $31 \pm 3$ & $22 \pm 8$ \\
\hline \multirow{3}{*}{ (c) Rayleigh } & A/a.u. & $3.5 \pm 0.8$ & $1.0 \pm 0.1$ & $2.9 \pm 0.3$ & $9 \pm 3$ \\
$\left(\sim \lambda^{-4}\right)$ & $\lambda / \mathrm{nm}$ & $624 \pm 4$ & $659 \pm 2$ & $696 \pm 2$ & $731 \pm 4$ \\
& $w_{\text {FWHм }} / \mathrm{nm}$ & $18 \pm 3$ & $22 \pm 2$ & $31 \pm 4$ & $27 \pm 5$
\end{tabular}

width at half maximum $\left(w_{\text {Fwнм }}\right)$ can differ by a factor of two for different scattering models. The constant considering the wavelength independent Mie scattering (b) was fitted to be $2 \pm 4$ times larger than the second feature at $655 \mathrm{~nm}$. The factor of proportionality considering Rayleigh scattering (c) was not given free for fitting but had to be set to a fixed value of $5.5 \times 10^{7} \mathrm{~nm}^{4}$ to make the fit converge.

Since all fit models match very well to the measured absorption data at hand, it is impossible to decide, which type is the dominant scattering mechanism or if scattering contributes to the optical loss at all.

Nonetheless, if periodic condensation or precipitation plays a role in the oscillation scheme, this is not necessarily restricted to drops or soot in the gas. On the chamber walls and, more importantly, on the surface of the mirrors a layer of material could be deposited. This could distort the results of the measurements for CRDS as well as IBBCEAS. The deposited layer would have an other refractive index than the gas mixture and leads to a change of the mirror reflectivity. The wavelength dependence of this effect, observed for dielectric mirrors in liquids, is 
very similar to that of the absorption features measured here. Although this process is also able to explain the observed phenomenon, it would impose a periodic condensation and evaporation of naphthalene or its reaction products on the mirrors, which is unlikely due to the constant temperature measured in the vacuum chamber and the time dependence of the absorption peaks (see section 5.7).

Due to the limited usable wavelength region of the laser dye available at the time, the reflectivity of the mirrors were measured with CRDS only in the region (636$688 \mathrm{~nm}$ ) marked by the black double arrow in Fig. 5.7. A polynomial of the $6^{\text {th }}$ order fitted to the measured reflectivity curve extrapolates the reflectivity to the regions left and right. The fitted constants are therefore only accurate for the second peak around $655 \mathrm{~nm}$. Since only half of the features around $619 \mathrm{~nm}$ and $736 \mathrm{~nm}$ were measured, the fitting parameters for these peaks are less accurate.

\subsection{IBBCEAS - Time Dependence of the Ab- sorption Features}

The temporal development of the absorption for type II responses is the same for the whole spectral region. In the case of the type I responses the features at $655 \mathrm{~nm}$ and $694 \mathrm{~nm}$ differ slightly, as shown in Fig. 5.8(a), where the absorption per pass $A$ at these wavelengths is shown as a function of time. At $655 \mathrm{~nm}$ the absorption rises slightly faster than at $694 \mathrm{~nm}$. This is indicated with a green arrow where the green data points, measured at the same time as the black ones, are always higher. After a maximum absorption has been reached the absorption at $694 \mathrm{~nm}$ decays slower (black arrow). This tiny effect is almost too small to be taken seriously, though it was observed repeatedly. The chemical system 1 proposed in Gash04 can be used as a model to explain the measured time dependence of the 


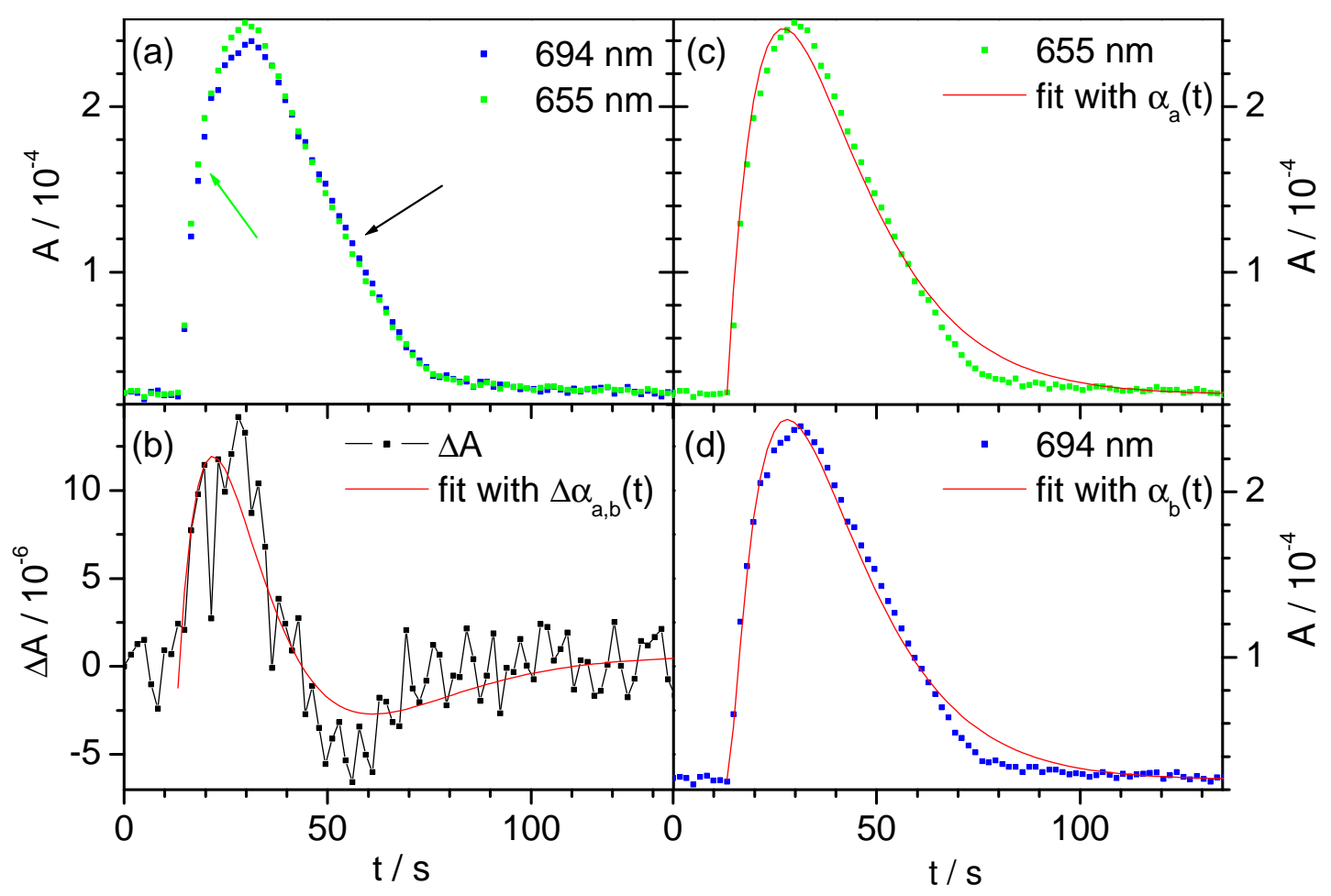

Figure 5.8: Temporal development of the absorption $A$ at $655 \mathrm{~nm}$ and $694 \mathrm{~nm}$ directly after 20 shots of UV multi-photon laser $\left(E_{\text {pulse }}=45 \pm 2 \mathrm{~mJ}\right)$ photolysis of naphthalene. (Other parameters see Fig. 5.6), (a) $A(t, 655 \mathrm{~nm})$ and $A(t, 694 \mathrm{~nm})$, (c and d) $A(t, 655 \mathrm{~nm})$ and $A(t, 694 \mathrm{~nm})$ fitted with $\alpha_{\mathrm{a}}(t)$ and $\alpha_{\mathrm{b}}(t)$, respectively, (b) $\Delta A(t)=A(t, 655 \mathrm{~nm})-$ $A(t, 694 \mathrm{~nm})$ fitted with $\Delta \alpha_{\mathrm{a}, \mathrm{b}}(t)=\alpha_{\mathrm{a}}(t)-\alpha_{\mathrm{b}}(t)$.

absorption of different chemical species. Following this model two reactants $\mathrm{Q}$ and $\mathrm{S}$ are produced by the multi-UV-photon excitation. They react to an intermediate compound $\mathrm{A}$ with a rate defined by the rate coefficient $k_{e}$. $\mathrm{A}$ is converted to a second intermediate species $\mathrm{B}$ which finally reacts to the product $\mathrm{C}$ with the rate coefficients $k_{u}$ and $k_{2}$ respectively. Refer to the appendix A.2 for a more detailed description of this chemical system. According to this model the concentration $a(t)$ of the intermediate A should rise and fall faster than the concentration $b(t)$ of the intermediate B. Since the absorption coefficients $\alpha_{a}$ and $\alpha_{b}$ are proportional to the concentrations of the species, the rate coefficients for the chemical reactions 
can be obtained by fitting the theoretical time response to the observed absorption behaviour.

The absorption at $655 \mathrm{~nm}$ raises and falls first and is therefore assigned to the species A while the feature at $694 \mathrm{~nm}$ is assigned to the species B. As shown in Fig. 5.8 (c) and (d) the fits of the absorption $\alpha_{a}$ and $\alpha_{b}$ represent the measured data poorly. According to the model in Gash04 which was used for the fit, the decay should be purely monoexponential. The measured absorption, however, decays linearly at first for $\approx 30 \mathrm{~s}$ and exponentially after that. This discrepancy could be due to the beginning oscillation of the background. For the explanation of an oscillation an additional reaction step of the scheme

$$
\mathrm{A}+2 \mathrm{~B} \rightarrow 3 \mathrm{~B} \quad \text { with a rate } \quad k_{1} a(t) b^{2}(t)
$$

had to be introduced Gash04. This autocatalytic feedback could disturb the exponential decay at the beginning of an oscillation. Nonetheless even the difference $\Delta \alpha_{a, b}=\alpha_{a}(t)-\alpha_{b}(t)$, shown in Fig. 5.8(b), behaves qualitatively in the same way as experimentally observed. Fits to several time dependent absorption measurements yielded a range of rate coefficients: $k_{e}=(0.07 \pm 0.03) s^{-1}$, $k_{u}=(0.07 \pm 0.03) s^{-1}$ and $k_{2}=(0.9 \pm 0.3) s^{-1}$ which match for $k_{e}$ and $k_{u}$ in the margins of error to the values obtained from the fit of $\Delta \alpha_{\mathrm{a}, \mathrm{b}}$ to the difference of the absorption $\Delta A$. A discrepancy exists for the value $k_{2}$ which was fitted to $(0.07 \pm 0.04) s^{-1}$. Due to the poor quality of the difference data as well as the poor match of the theoretical with the experimental curves, the agreement is only qualitatively. According to the fit results the cross-section $\sigma_{a}$ of the species A seems to be an order of magnitude larger than for $\sigma_{b}$ of the species B. But since the fit values obtained for $\sigma_{a}$ and $\sigma_{b}$ show a strong dependence on other fit parameters and vary about 2 orders of magnitude for different data sets, the cross-sections are essentially rough estimates. 
The differences in the temporal behaviour of the absorption peaks can be observed only for short raise and decay times directly after a UV-photolysis. The oscillating absorption, however, changes on a larger time scale and due to the smaller gradient the differences in the temporal behaviour can not be distinguished anymore from the noise.

Nonetheless the different temporal behaviour for different absorption features represent an argument against the periodic condensation at the mirror surfaces as discussed above. Since the refractive index for a molecule layer should be the same during condensation and evaporation, the temporal behaviour of the reflectivity should not differ for different wavelengths.

\subsection{IBBCEAS - Comparison to known spectra}

A comparison of the measured absorption spectrum to known spectra should indicate which of the molecules in a long list of potential species formed Gash04] can be excluded as carrier of the oscillating absorption. In Fig. 5.9 spectra of the naphthalene cation (b) and azulene (c) are shown in comparison to the measured oscillating absorption (a), fitted gauss functions and a wavelength independent background.

The naphthalene cation $\left(\mathrm{C}_{10} \mathrm{H}_{8}{ }^{+}\right)$is very likely to be formed by a multi photon excitation at $308 \mathrm{~nm}$. It is improbable that the electric charge it carries can exist in a steel chamber for the time of the oscillation, but nonetheless it is one of the important intermediates. Its spectra measured in arbitrary units in an argon matrix [Salama91] and in a supersonic jet Biennier03] respectively were reconstructed with the data given in the publications and are shown in Fig. 5.9(b). Even though $\mathrm{C}_{1} 0 \mathrm{H}_{8}^{+}$has absorption lines around $615 \mathrm{~nm}$ ans $654 \mathrm{~nm}$, which would 


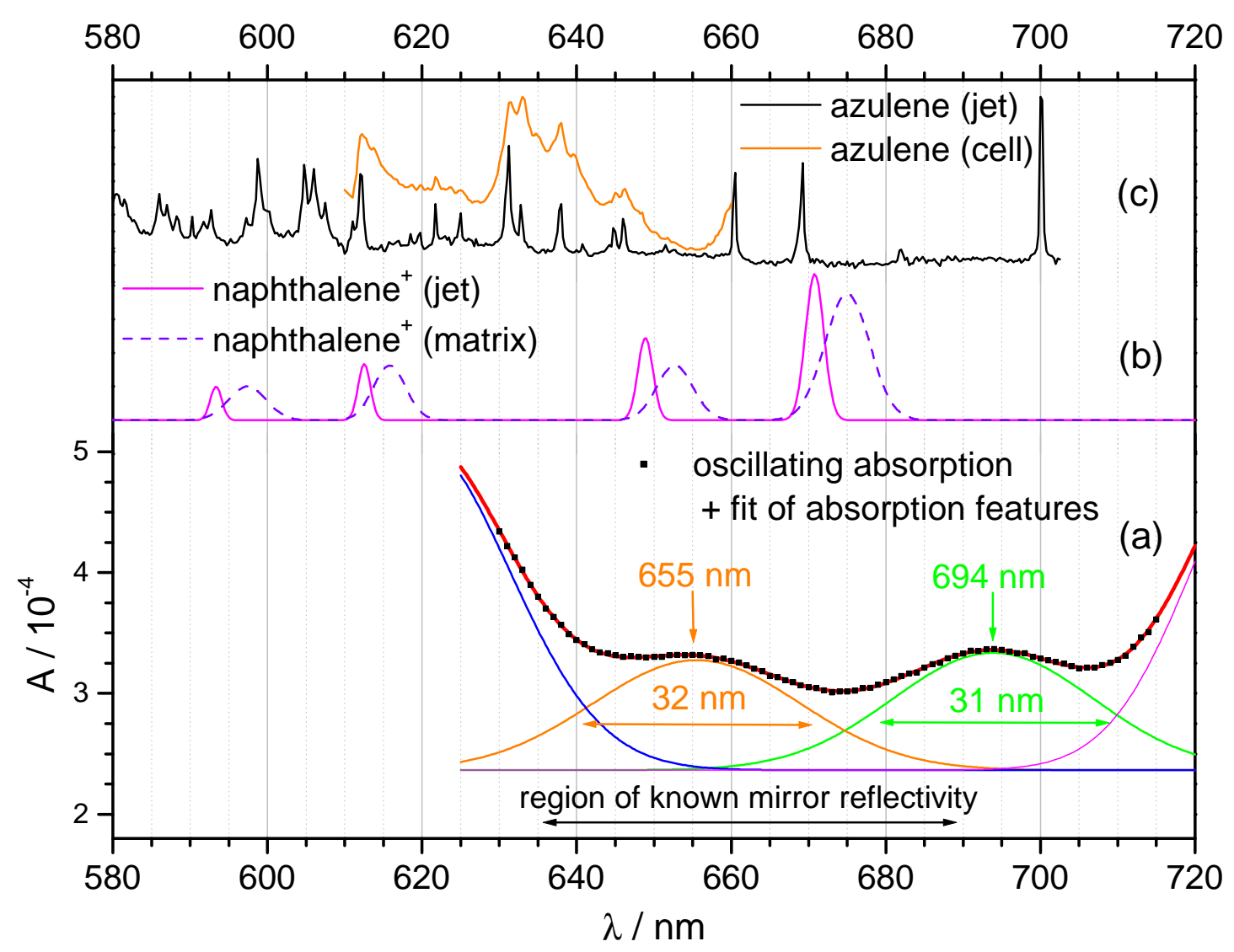

Figure 5.9: (a) Absorption spectrum of an oscillation maximum (black dots, only every $4^{\text {th }}$ measured point is shown) together with fits of the absorption features. (b) Spectrum of the naphthalene cation measured in a supersonic jet [Biennier03] and in an argon matrix [Salama91] (c) Spectrum of azulene measured in a supersonic jet [Ruth99] and in a gas cell [Ruth02a].

match features in our experiment, it has also a line around $675 \mathrm{~nm}$ where no absorption feature was measured. Hence the naphthalene cation is not the carrier of the oscillating absorption.

Azulene $\left(\mathrm{C}_{10} \mathrm{H}_{8}\right)$ is a stable isomer of naphthalene containing a five member and a seven member ring. In [Suzuki96] a conversion from naphthalene to azulene by a 2 UV-photon (193 nm) absorption buffered in a 1000 mbar nitrogen atmosphere has been reported. In Cui00 the potential energy for the cationic naphthalene/azulene 


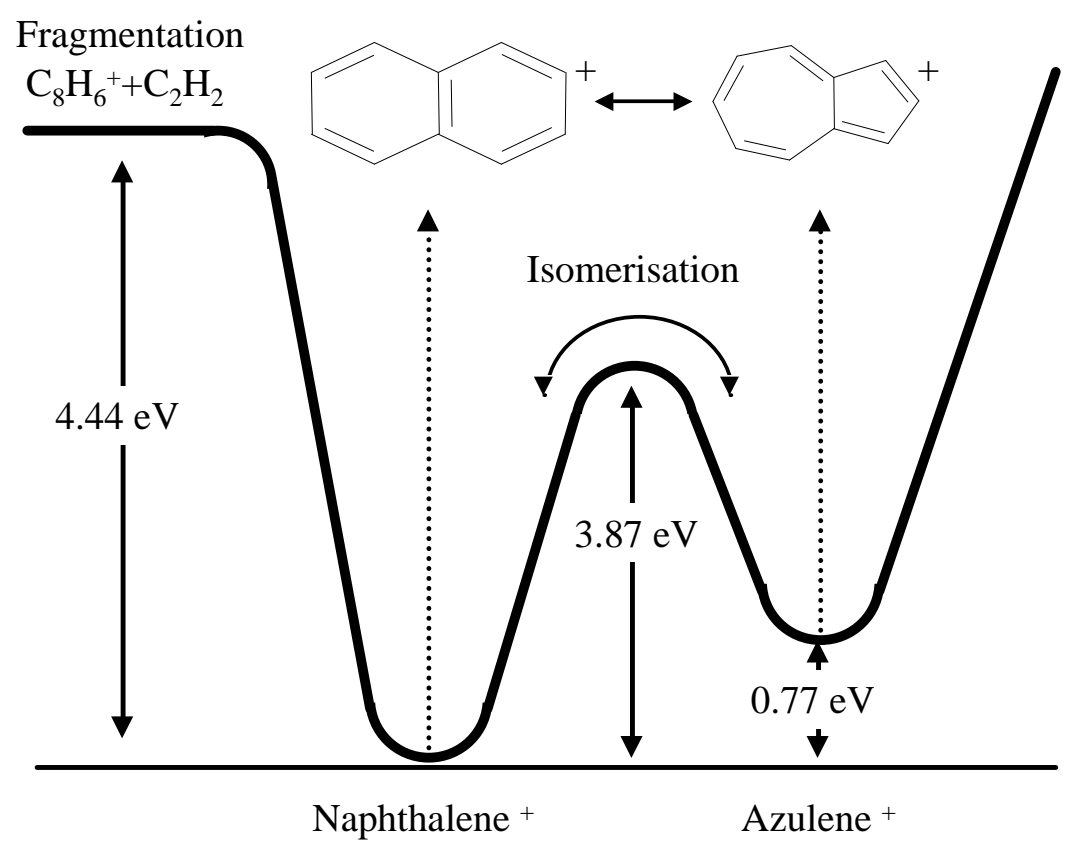

Figure 5.10: Schematic energy potential diagram for the isomerisation and fragmentation of naphthalene- and azulene-cation radicals Cui00.

isomerisation could be determined (see Fig. 5.10). The isomerisation takes place below the energy required for the dissociation of the ion by $\mathrm{C}_{2} \mathrm{H}_{2}$ loss. Under the experimental conditions for which oscillations can be observed it is likely that this isomerization occurs during the UV laser shots. However a comparison of the azulene spectra to the oscillating absorption shows that azulene is not observable: In Fig. 5.9 (c) azulene spectra are shown for a measurement in a supersonic jet [Ruth99] and for one in a gas cell at the vapour pressure of the room temperature [Ruth02a]. The absorption line at $669 \mathrm{~nm}$ of the jet spectrum can not be seen in the oscillating spectrum. Either the amount of azulene produced is too small to be detectable or azulene is not produced due to photo dissociation processes which can dominate for special photon energies Allain96. 
Absorption of four photons, corresponding to a total excitation energy of $16.1 \mathrm{eV}$, could cause the naphthalene molecule to fragment. The benzene ion $\left(\mathrm{C}_{6} \mathrm{H}_{6}{ }^{+}\right)$is one of the possible products appearing at the average energy of $15.7 \mathrm{eV}$ [Jochims92]. The benzene ion is known [Miller80] to have several absorption lines at $552.5 \mathrm{~nm}$ and below but none in the wavelength region of interest.

The electronic transition of benzene $\left(\mathrm{C}_{6} \mathrm{H}_{6}\right)$, as a result of the recombination of the benzene ion with an electron, is in the UV region. In the visible region there are only very weak $\mathrm{CH}$ stretch overtones Reddy82. However, the line positions at $710.5 \mathrm{~nm}, 604.0 \mathrm{~nm}$ and $529.0 \mathrm{~nm}$ for the fourth, fifth and sixth overtone, respectively, do not match with the positions of the oscillating absorption features presented in this work.

In this work only a few selected known spectra of stable species, probably produced by multi UV photon absorption of naphthalene, were compared with the oscillating absorption spectrum. The molecules however which could be formed under these special experimental conditions are numerous, since apart from the possible formed fragments $\left(\mathrm{H}, \mathrm{C}_{2} \mathrm{H}_{2}, \mathrm{C}_{4} \mathrm{H}_{2}, \mathrm{H}_{2}\right.$ and $\left.\mathrm{C}_{3} \mathrm{H}_{3}\right)$ most of the products are ions $\left(\mathrm{C}_{10} \mathrm{H}_{7}^{+}, \mathrm{C}_{8} \mathrm{H}_{6}^{+}, \mathrm{C}_{6} \mathrm{H}_{6}^{+}, \mathrm{C}_{1} 0 \mathrm{H}_{6}^{+}, \mathrm{C}_{7} \mathrm{H}_{5}^{+}, \mathrm{C}_{8} \mathrm{H}_{5}^{+}, \mathrm{C}_{6} \mathrm{H}_{4}^{+}, \mathrm{C}_{6} \mathrm{H}_{5}^{+}, \mathrm{C}_{3} \mathrm{H}_{3}^{+}, \mathrm{C}_{4} \mathrm{H}_{4}^{+}\right.$, $\mathrm{C}_{5} \mathrm{H}_{3}^{+}, \mathrm{C}_{7} \mathrm{H}_{3}^{+}, \mathrm{C}_{4} \mathrm{H}_{2}^{+}, \mathrm{C}_{6} \mathrm{H}_{3}^{+}$and $\mathrm{C}_{4} \mathrm{H}_{3}^{+}$, respectively) [Jochims92 or radicals and chemical reactions among them or with naphthalene are very likely to happen.

Even if none of the produced electrons recombines with an ion, the lifetime of the ions should be less than the diffusion time $(\sim 10 \mathrm{~s}$, Gash04] $)$ to the walls of the steel chamber. Thus, the amount of ions in the chamber is too small to be detected.

An estimation of the number of naphthalene molecules in the photolysis region indicates that the oscillating absorption $\left(\alpha \sim 10^{-5} \mathrm{~cm}^{-1}\right)$ is not only due to primary photolysis products since the maximum concentration $\left(2.3 \times 10^{6}\right.$ molecules $\left./ \mathrm{cm}^{3}\right)$ 
obtainable from photolysis is too low for such strong absorptions, assuming typical absorption cross-section for allowed transitions of $\sigma \sim 10^{-17}$ to $10^{-16} \mathrm{~cm}^{2}$. This could explain why neither azulene nor benzene absorption structures are observable in the IBBCEA spectrum. It is more likely that the species produced at the multiUV-photon excitation is a catalyst for a reaction of naphthalene, which is present in the chamber abundantly.

\subsection{Summary}

The complex oscillatory behaviour of a recently discovered closed gas-phase system was further investigated. Employing the incoherent broad-band cavity-enhanced absorption technique, the optical absorption spectrum of static naphthalene vapour buffered with a noble gas at room temperature was measured between 630 and $715 \mathrm{~nm}$ (resolution: $1.7 \mathrm{~nm}$ ) as a function of time (time resolution: $1.65 \mathrm{~s}$ ). No other known oscillatory system is triggered by a multi-UV-photon (at $308 \mathrm{~nm}$ ) excitation process, as it is for the system presented here.

A comparison of the measured spectra with known spectra of selected molecules, suspected to be involved in the oscillation process, shows that the carrier of the oscillating absorption is neither naphthalene $\left(\mathrm{C}_{10} \mathrm{H}_{8}\right)$, nor the naphthalene cation $\left(\mathrm{C}_{10} \mathrm{H}_{8}{ }^{+}\right)$, nor azulene $\left(\mathrm{C}_{10} \mathrm{H}_{8}\right)$, or benzene $\left(\mathrm{C}_{6} \mathrm{H}_{6}\right)$ nor its cation $\left(\mathrm{C}_{6} \mathrm{H}_{6}{ }^{+}\right)$.

The absorption features in the spectrum are very smooth and broad $(\sim 30 \mathrm{~nm})$. The spectra were evaluated considering a contribution of Mie scattering, Rayleigh scattering and no scattering at all. All three fit functions matched the measured data and the question if scattering contributes to the measured optical losses by formation of liquid droplets or solid particles floating in the chamber could therefore not be ruled out entirely. 
Droplets condensing on the mirrors could however be ruled out, since between two observed absorption features, measured directly after an multi photon excitation, a very small temporal delay $(\sim 1 \mathrm{~s})$ was detected. This could be interpreted as evidence, that at least two different species with different absorption peaks at $655 \mathrm{~nm}$ and $694 \mathrm{~nm}$, respectively, are involved in the oscillation process.

The estimated number of molecules primarily formed in the photolysis of naphthalene is too small to explain the magnitude of the absorption oscillation. They are more probably catalysts for a chemical reaction, which forms the absorbing species.

\subsection{Future Work}

In future experiments the observation of a larger spectral range is necessary, i) to improve the accuracy of the line positions, widths and amplitudes obtained by a fit, ii) to decide which scattering process, or if any at all, contributes to the oscillating absorption, iii) to identify more oscillating absorption features for improved and more reliable comparisons to known spectra and iv) to monitor in multiplex operation the unknown oscillating species, the concentration of possible photolysis products as well as the naphthalene concentration. The UV spectrum of naphthalene would give information not only about the concentration and the vapor pressure of naphthalene at the beginning of the experiment but also about the temporal behaviour during the oscillation.

An improved time resolution would be useful to measure more accurately the temporal shifts between absorption features due to the oscillating concentration of different intermediates. 
A simultaneous measurement of spectra for several absorption paths at different distances from the UV laser excitation focus could give information about the spatial distribution of the absorbing species as a function of time. This would help to determine whether the absorbing species is subject to slow transport phenomena like diffusion, convection or even gravity.

Up to now all information about this gas phase system were gathered by the measurement of the absorption response the system to given experimental conditions. Other methods could provide additional information: (i) For instance time resolved mass spectroscopy could determine probable candidates of species involved and hence reduce the number of necessary comparisons of spectra to the measured data. Due to the relatively high pressure in the chamber and the strong influence of small changes of the setup this setup could however prove to be problematic. (ii) A chemical analysis of molecules gathered in the chamber after an oscillation experiment would possibly identify the final product(s) of the reaction chain.

Azulene as an isomer of naphthalene could prove to show a similar oscillation behavior and should be studied under the same conditions. The $\mathrm{S}_{0} \rightarrow \mathrm{S}_{1}$ absorption origin of azulene is however around $700 \mathrm{~nm}$ and several vibronic absorption lines can be found in the region where the oscillation was observed up to now. Therefore it has to be checked first, if oscillating absorption features can be also found in the wavelength region well above $700 \mathrm{~nm}$, where azulene shows no absorption. A distinction between the contribution of azulene and the oscillating species to the absorption could otherwise be very difficult. 


\section{Chapter 6}

\section{Azulene in a Supersonic Jet}

A species in a supersonic jet can be studied under conditions where pressure and Doppler broadening as well as the number of excited vibrational and rotational energy levels is strongly reduced. Therefore the analysis and interpretation of spectra of molecules taken in a supersonic jet is much simplified. However the density of the species in the jet and thus the absorption coefficient are very low. Only ultra-sensitive techniques are able to obtain absorption spectra with a satisfactory signal-to-noise ratio.

The cooling effect in a supersonic jet is due to gas being expanded from a high pressure reservoir along a large pressure gradient through a nozzle (orifice diameters are typically between 0.1 and $1 \mathrm{~mm}$ ) into a low pressure vacuum chamber. Due to a very large number of collisions in, and immediately after the nozzle the gas is cooled down. Very low translational temperatures $(\sim 1 \mathrm{~K})$ of the adiabatically expanded gas can be achieved and the gas particles become virtually isolated in the jet stream formed. The velocity of the expanding gas flow into the vacuum is thereby higher than the speed of sound in the gas at the expansion temperature. A supersonic jet may be divided into five zones at different distances $x$ from the 


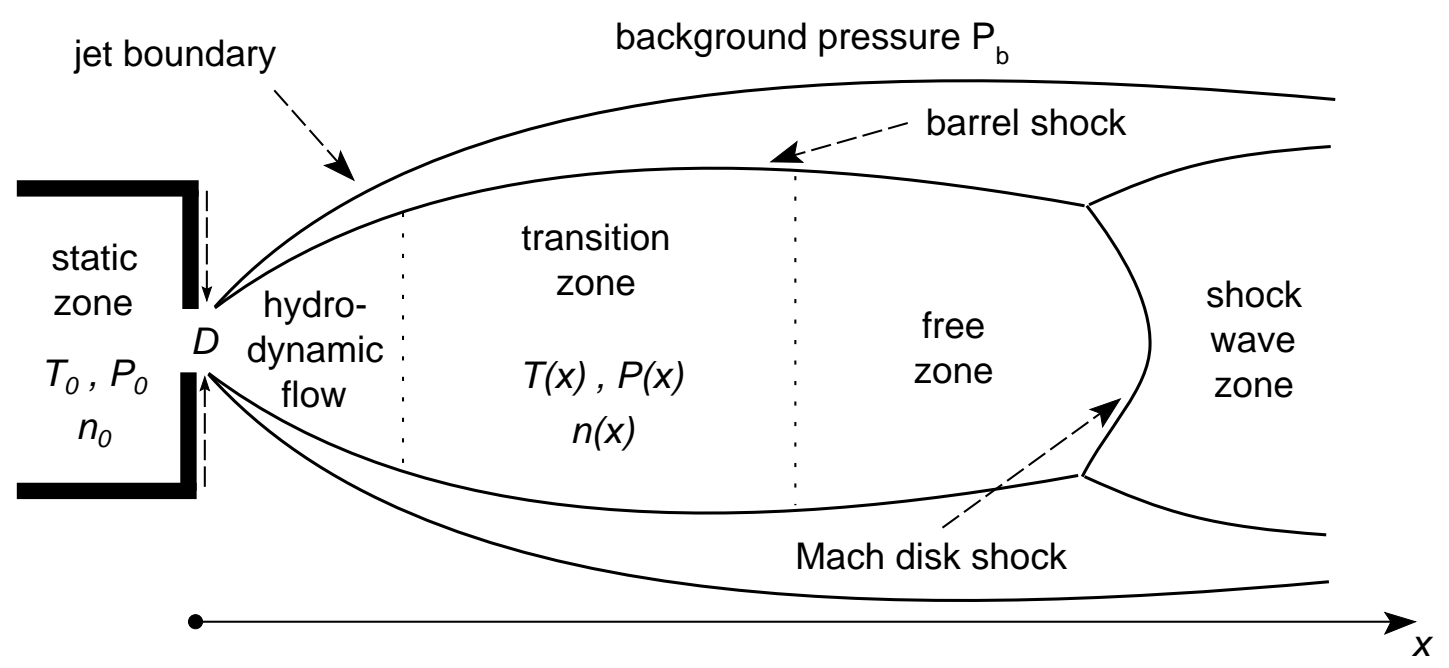

Figure 6.1: Scheme of the five zones in a adiabatic continuum jet expansion. (1) static: Before expansion at a "high" pressure $P_{0}$ with a molecule density $n_{0}$ and a temperature $T_{0}$, (2) hydrodynamic, (3) transition: expanding in the vacuum, reducing $T(x), P(x)$ and $n(x)$ with the distance $x$ from the nozzle with the orifice diameter $D,(4)$ free: molecules effectively isolated (5) shock wave zone due to the rest gas pressure $P_{b}$.

nozzle orifice (diameter $D$ ) as shown in Fig. 6.1. (1) The reservoir, or static zone, which is basically a static cell containing the carrier gas and the seed molecule vapour; (2) the region of hydrodynamic flow directly in and behind the nozzle $(x<4 D)$, in which the gas rapidly cools as it expands adiabatically; (3) the transition region, where the gas molecules have been cooled to a few Kelvin, but still undergo relatively frequent collisions and continue to accelerate; (4) the free zone, where the molecules have reached a terminal velocity and are effectively isolated $(x>20 D)$; and $(5)$ the shock wave zone behind the Mach disk shock, where the free supersonic jet conditions are destroyed by shock waves carried by the background gas in the chamber. The distance $x_{m}$ of the Mach disk from the nozzle depends on the pressure $P_{0}$ of the gas in the nozzle and the pressure $P_{b}$ of the background gas in the chamber $\left(x_{m}=0.67 D\left(P_{0} / P_{b}\right)^{1 / 2}\right)$ [Miller88].

The gases generally used in supersonic expansions are typically noble gases such as helium or argon. Of particular interest are so-called seeded beams, where poly- 
atomic molecules are mixed into the carrier gas. The seed molecules attain a very similar translational temperature to the carrier gas.

The number of inter-particle collisions required to reach thermal equilibrium between the seed and the carrier molecule increases with the number of degrees of freedom of the molecules in the expansion [Lambert77]. During the expansion process, due to the decreasing number density the collision rate is lowered to such an extent that an equilibrium cannot be reached. The different rotational, vibrational and translational degrees of freedom of the seed (and carrier) molecules before the expansion are on average converted by collisions into translational degrees of freedom in the jet with different probability.

For this reason, the jet cannot be characterized by a single temperature. In fact, four temperatures are required to describe the occurring energy distribution: The translational temperatures $T_{\operatorname{trans}(\mathrm{c})}$ and $T_{\text {trans(s) }}$ of the carrier atoms and seed molecules, respectively, the rotational temperature $T_{\text {rot(s) }}$ of the seed molecules and their vibrational temperature $T_{\mathrm{vib}(\mathrm{s})}$. The temperatures in most seeded supersonic jets are generally in reversed order to the energy conversion cross-sections:

$$
T_{\text {trans(c) }}<T_{\operatorname{trans}(\mathrm{s})}<T_{\operatorname{rot}(\mathrm{s})}<T_{\operatorname{vib}(\mathrm{s})}
$$

Typical orders-of-magnitude of temperatures for large aromatic seed molecules in pulsed supersonic jets are: $T_{\operatorname{trans}(\mathrm{s})} \sim 1 \mathrm{~K}, T_{\text {rot(s) }} \sim 10 \mathrm{~K}$ and $T_{\mathrm{vib}(\mathrm{s})} \sim 100 \mathrm{~K}$.

Due to the low temperatures in supersonic expansions the formation of weakly bound complexes is possible. The bond energies are based on dipolar interactions which often are so weak that the complex would not be stable at room temperature. Van der Waals complexes are typical, consisting of e.g. the carrier noble gas alone (He-He, Ne-Ne, Ar-Ar, Kr-Kr) or in the presence of seed molecules form a (noble gas)-(seed molecule) complex. Particularly interesting are cluster systems 
consisting of more than two atoms or molecules, since they represent the transition state of matter between isolated atoms and the bulk, forming a solid. The rate of formation depends strongly on the temperature $T_{\text {trans }}$, the local pressure and the vapour pressure of the (seed) species of interest. Although in the last years intensely investigated for a better understanding of the weak molecular interactions, cluster formation is however not desired for spectroscopic studies of isolated molecules.

For a comprehensive treatment of supersonic jets several excellent texts are available like for instance [Miller88, Hollas95, Anderson74, Fluendy73.

\subsection{Motivation}

High resolution laser spectroscopy of cold molecules is one of the main research fields of the research group of Hese. Several experiments in the supersonic jet with methods like cavity-ring-down spectroscopy or optical optical double resonance (OODR) were performed [Ruth99, Hoheisel03]. To prepare the setup for a measurement, a lot of time and sample molecules have to be invested: The absorption features at very low temperatures of the order of a few Kelvin are very narrow $\left(\sim 1 \mathrm{~cm}^{-1}\right)$ and therefore not easy to find with a dye laser $\left(\sim 0.1 \mathrm{~cm}^{-1}\right)$. Also rarely all of the absorption features are scanned for different experimental parameters to optimize the signal obtained, due to reason of time. Distortions of the absorption features, for instance because of temperature changes or cluster formation, are therefore seldom noticed.

A sensitive broad-band technique to measure small absorptions is therefore very useful to find the optimal experimental parameters and to obtain vibronic spectra 
before a high resolution investigation of the rovibronic absorption structure is performed. This could be quite useful for the detection of new species or ions.

The motivation to investigate the $\mathrm{S}_{1} \leftarrow \mathrm{S}_{0}$ absorption of azulene in a continuous jet using IBBCEAS is based on previous work by [Ruth99] where this transition was studied in a pulsed supersonic expansion by means of conventional pulsed CRD spectroscopy. The experimental data obtained in the region between 14275 and $17300 \mathrm{~cm}^{-1}$ indicated a conical intersection (see section 6.2) of the $\mathrm{S}_{0}$ and the $\mathrm{S}_{1}$ potentials at $\approx 2100 \mathrm{~cm}^{-1}$ excess energy above the band origin at $14283.3 \mathrm{~cm}^{-1}$. However, the spectral region far above the conical intersection, was not addressed in [Ruth99]. In this region the lifetimes are expected to be extremely short ( $\ll 1 \mathrm{ps})$ due to the very rapid internal conversion $S_{1} \sim S_{0}$.

The measurements presented in this section demonstrate, that the resolution and the sensitivity of IBBCEAS are sufficient to obtain vibrational spectra of larger molecules in a supersonic jet, which are comparable to the quality of CRD spectra. As an example for the potential to optimize experimental parameters with IBBCEAS, the influence of the argon pressure is discussed. The $\mathrm{S}_{1} \leftarrow \mathrm{S}_{0}$ absorption spectrum of azulene is measured for the first time over the broad spectral range between 17300 and $20255 \mathrm{~cm}^{-1}$, a region well above the conical intersection of the $\mathrm{S}_{0}$ and $\mathrm{S}_{1}$ potentials. The emphasis of the discussion of the spectrum will be on the highly congested vibronic structure and the spectral background.

\subsection{The Conical Intersection}

A conical intersection is an $n-2$ dimensional subspace of the $n$ nuclear coordinates called intersection space. The potential energy $E$ of the two states $\left(\mathrm{S}_{0}\right.$ and $\left.\mathrm{S}_{1}\right)$ remains the same at any point in this space; the degeneracy is only lifted when 


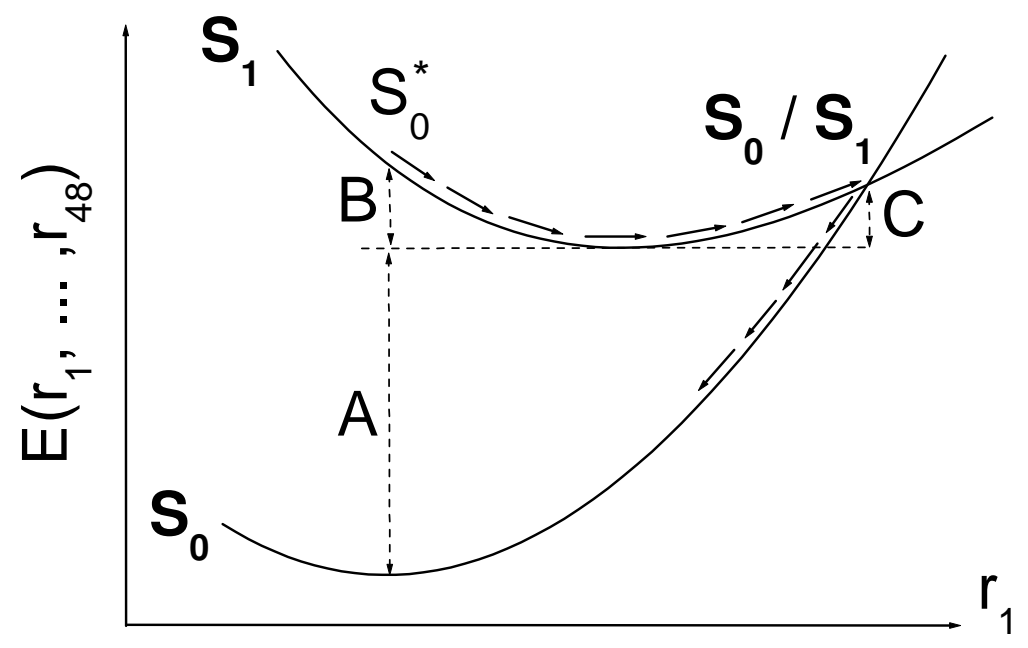

Figure 6.2: Scheme of the conical intersection of the $\mathrm{S}_{0}$ and $\mathrm{S}_{1}$ potential surfaces of azulene as a function of the normal coordinate $r_{1}$.

the molecule is distorted along the two remaining linearly-independent nuclear coordinates. Conical intersections have been extensively discussed in the literature and investigations have indicated that they can be a common feature in organic systems (see references 13, 14 and 15 in Bearpark96).

In the case of azulene an unavoided crossing of the $S_{0}$ and $S_{1}$ potential surfaces is located a few thousand $\mathrm{cm}^{-1}$ above the azulene $\mathrm{S}_{1}$ minimum. After an excitation to the $\mathrm{S}_{1}$ state by the energy $A+B$ the molecule travels along the normal coordinate $r_{1}$ from the $\mathrm{S}_{0}$ Franck-Condon geometry $\left(\mathrm{S}_{0}^{*}\right)$ through a minimum to an $\mathrm{S}_{1} / \mathrm{S}_{0}$ sloped conical intersection (see Fig. 6.2). If the energy $\mathrm{C}$ is smaller than $\mathrm{B}$ the molecule can decay without radiation to high vibrational $S_{0}$ states.

This conical intersection is the lowest energy point on a 46-dimensional hyperline (a subspace of 48 nuclear normal coordinates $r_{i}$ ) where the $\mathrm{S}_{1}$ and $\mathrm{S}_{0}$ states cross. A simulation presented in Bearpark96 suggests that the radiationless decay process at the crossing can take place before a single $\mathrm{S}_{1}$ oscillation is completed and that 
the return to the ground state is therefore expected to occur on the femtosecond time scale, independent of $\mathrm{S}_{1}$ vibrational cooling or equilibration.

\subsection{Experimental Setup}

The experimental setup for the application of the IBBCEAS technique to supersonic jets is schematically shown in Fig. 6.3. Azulene (melting point $\sim 99^{\circ} \mathrm{C}$ ) was heated in a quartz oven to temperatures between 95 and $125^{\circ} \mathrm{C}$, and seeded into the carrier gas argon at stagnation pressures between 300 and 1000 mbar. The mixture was expanded through a quartz nozzle with an orifice of $200 \mu \mathrm{m}$ into a high vacuum. The vacuum chamber with a volume of $\approx 200$ litre was pumped by two diffusion pumps (6000 and 3000 litre/s) keeping the pressure in the vessel lower than $2 \times 10^{-4}$ mbar at all times. Azulene molecules were trapped with copper plates, which were cooled with liquid nitrogen and mounted in a position where the seeded jet collided with the chamber walls. The supersonic jet intercepted the optical axis of the ring-down cavity at a distance of $13 \mathrm{~mm}$ from the nozzle. This distance is large enough to probe free isolated molecules $(x>4 D=800 \mu \mathrm{m})$ and small enough to be well before the shock wave region of the Mach disk at $x_{m}=0.67 D\left(P_{0} / P_{b}\right)^{1 / 2} \approx 160 \mathrm{~mm}$ (see Fig. 6.1).

The optically stable cavity with a length of $\ell=70 \mathrm{~cm}$ was formed by two highly reflecting dielectric mirrors (plano-concave, $25 \mathrm{~mm}$ diameter), which vacuum-sealed the chamber and were adjustably mounted. Three sets of mirrors (REO, Boulder Colorado) with curvatures of $|r|=100-200 \mathrm{~cm}$ and reflectivities of $R \sim 0.9999$ around the centre wavelengths of 510, 560 and $610 \mathrm{~nm}$ were used to cover the entire spectral region studied. A graph which illustrates the regions of high reflectivity for these mirrors can be found in Appendix A.1. 


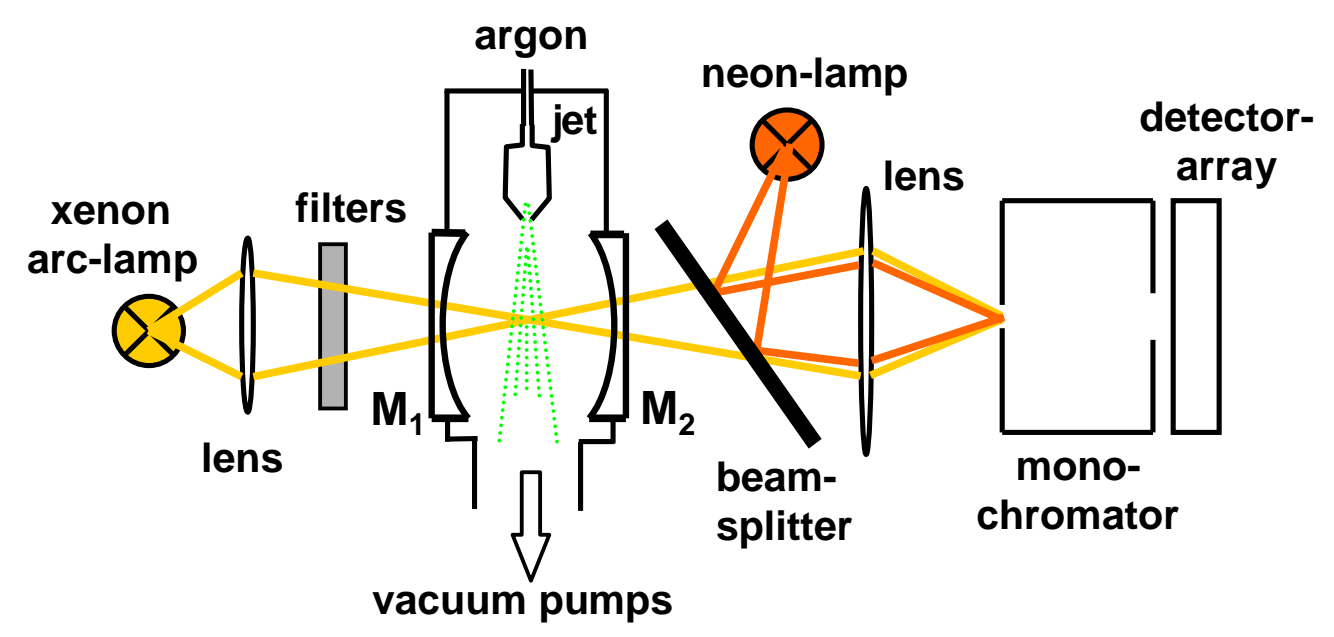

Figure 6.3: Schematically experimental setup for IBBCEAS in a supersonic jet.

The white-light emitted by a short-arc xenon lamp (ISAS Berlin) was spectrally narrowed by appropriate optical filters to the high reflectivity wavelength range of the respective mirrors. A set of lenses imaged the brightest zone of the arc lamp $(\sim 150 \mu \mathrm{m})$ into the mechanically not stabilized cavity as shown in Fig. 6.3. The light transmitted by the cavity was coupled into a light-guide with a circular input aperture and rectangular output aperture (width: $100 \mu \mathrm{m}$ ). The light guide was connected to the entrance slit of a grating monochromator (B\&M Spektronik, 2400 grooves $/ \mathrm{mm}$, spectral resolution $\approx 0.12 \mathrm{~nm}\left(\approx 4 \mathrm{~cm}^{-1}\right.$ at $\left.\left.550 \mathrm{~nm}\right)\right)$ which was used to disperse the light. Optionally the emission of a neon-lamp was reflected by a beamsplitter such that it entered the light-guide at the same angle as the light transmitted by the cavity. It was used to calibrate the wavelength for the spectra. The dispersed light was detected and integrated over time by a cooled and intensified photodiode-array detector (IRY-700S/BP-46, Princeton Instruments, Inc.), which was interfaced to a computer for further analysis of the data. The integration times were between $100 \mathrm{~s}$ and $1200 \mathrm{~s}$. The wavelength range of ca. $14 \mathrm{~nm}$ per integration period was optimized for the highest spectral resolution attainable with the limited detection area of 700 photodiodes $/ 18 \mathrm{~mm}$. 
Instead of the absorption coefficient, $\alpha$, the absorbance per pass $A=\alpha d$ was evaluated, because the distribution of azulene in the jet and hence the absorption path length $d$ was not exactly known. Using Eq. 2.16 and assuming $R=R_{1}=R_{2}$, $A$ is given by

$$
A=\alpha d \approx\left(\frac{I_{0}}{I}-1\right)(1-R)
$$

where $I$ and $I_{0}$ represent the detected intensity with and without azulene present in the jet, respectively. Both intensities were corrected for dark counts. It turned out that, despite the liquid nitrogen trap inside the vacuum chamber, vestiges of hot azulene in the rest gas were still detectable due to the fact that the interaction path for hot azulene molecules along the full cavity length $(\ell \approx 70 \mathrm{~cm})$ is much larger than the absorption path $d \sim 1-2 \mathrm{~cm}$ of cold azulene in the jet. For the measurement of $I_{0}$, the seeded jet was not turned off but instead moved completely off the optical axis of the ring-down cavity. In that way, the absorption of the residual hot azulene was approximately the same for the measurements of $I_{0}$ and $I$. Hence, the effect of hot azulene on the absorbance was well compensated with a maximum error of less than $10 \%$.

In this section, $A$ is given in units of $(1-R)$ since the reflectivities $R$ of the mirrors were not established for the given experimental conditions. Since $R$ is only slightly smaller than one, $A$ is quite sensitive to variations of $R$, particularly in wavelength regions where the high reflectivity of the cavity mirrors decreases rapidly. Therefore, the centre spectral range of high reflectivity was always used for measurements with a particular set of mirrors. Nevertheless, if $R(\lambda)$ is not known and if the wavelength region studied largely exceeds the high reflectivity spectral range of one set of cavity mirrors (in other words, if spectral ranges have to be concatenated), then the relative intensities cannot be accurately determined. By CRDS measurements it is know that the reflectivity curve of single stack mirrors, like they were used for this experiment, is without ripples and quite smooth in the 
high reflection region. Therefore the position and width of absorption lines can be accurately measured by the IBBCEAS technique despite the unknown reflectivity curves.

\subsection{IBBCEAS in Comparison to CRDS Measurements}

In order to demonstrate the viability of the IBBCEAS approach for the application of absorption measurements of species in a supersonic jet, the absorption of azulene

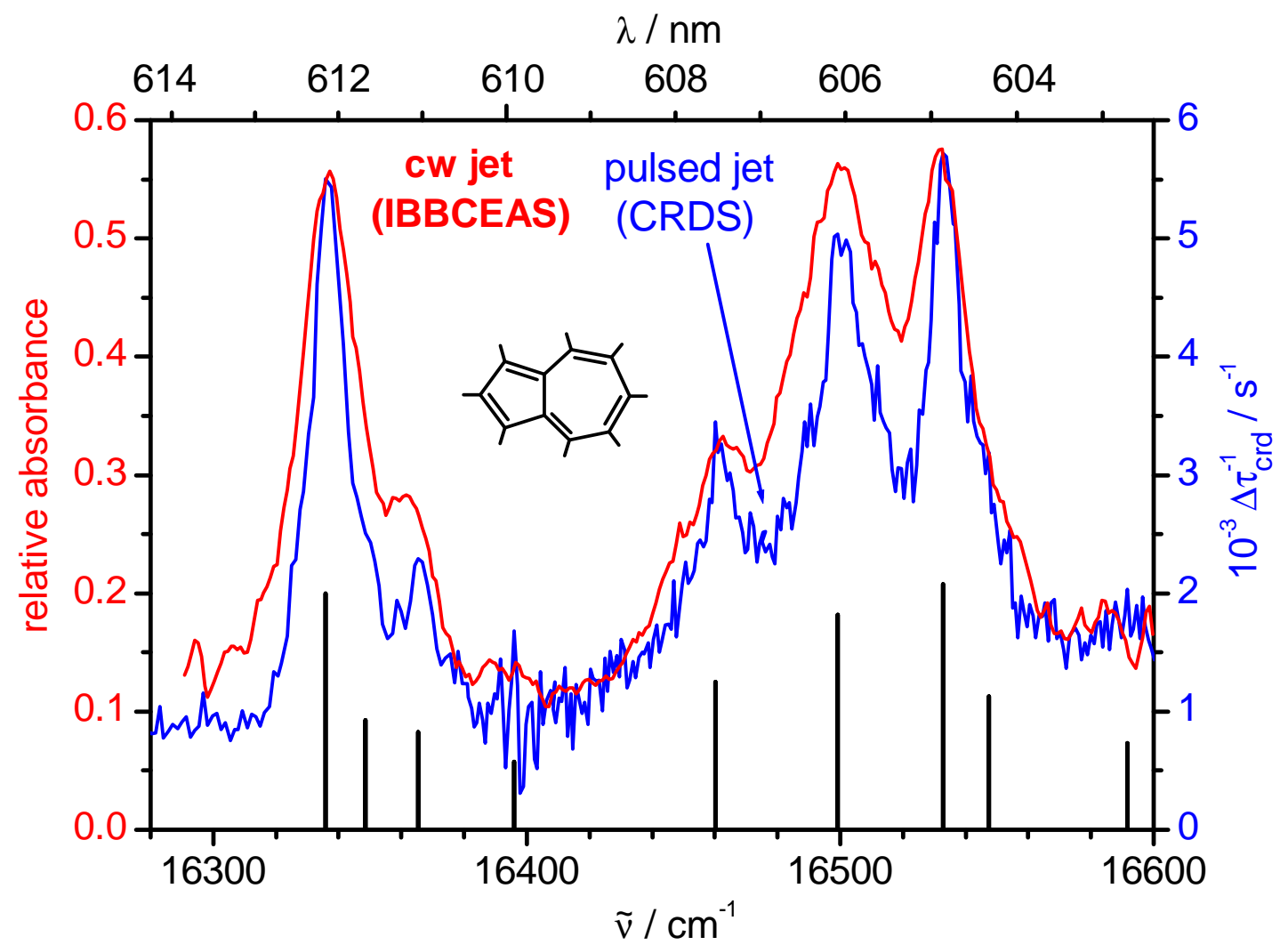

Figure 6.4: $\mathrm{S}_{1} \leftarrow \mathrm{S}_{0}$ absorption spectrum of jet-cooled azulene in the region of the $\mathrm{S}_{1} / \mathrm{S}_{0}$ conical intersection measured with IBBCEAS and pulsed CRDS [Ruth99. 
was measured in a known region of vibronic $\mathrm{S}_{1, \nu} \leftarrow \mathrm{S}_{0}$ transitions between 16224 and $16611 \mathrm{~cm}^{-1}$. The IBBCEA spectrum was measured with an exposure time of $100 \mathrm{~s}$ at an argon stagnation pressure of $300 \mathrm{mbar}$ and an oven temperature of $117 \pm 3^{\circ} \mathrm{C}$. It is shown in Fig. 6.4 in comparison to existing CRD data Ruth99.

The original absorption values in units of $(1-R)$ at 16336 (line maximum) and $16422 \mathrm{~cm}^{-1}$ (background) were used to adapt the IBBCEA spectrum to the CRD spectrum for better comparison. Hence, the left axis is expressed in terms of relative absorbance, the right axis refers to the pulsed CRD experiment, where the quantity $\Delta \tau_{\mathrm{CRD}}^{-1}$ represents the difference of reciprocal ring-down times $\tau_{\mathrm{CRD}}$ measured with the jet on and off ( $\Delta \tau_{\mathrm{CRD}}^{-1}$ is directly proportional to the absorption coefficient $\alpha$ ). The absorption features in Fig. 6.4 are rotational contour bands, which are due to overlapping homogeneous line widths $\left(>10 \mathrm{~cm}^{-1}\right)$ of rovibronic $\mathrm{S}_{1, \nu}$ states. The stick spectrum shows the position of vibronic absorptions identified in [Ruth99].

In good approximation, Lorentzian profiles can be fitted to the rotational contours (empirical approach) and the six most prominent features were described in that

Table 6.1: Comparison of the IBBCEA and CRD measurement of the six most prominent features in Fig. 6.4.

\begin{tabular}{ccc|ccc}
\hline \multicolumn{3}{c}{ IBBCEAS } & \multicolumn{3}{c}{ CRDS [Ruth99] } \\
$\tilde{\nu}_{\text {abs }} / \mathrm{cm}^{-1}$ & $\Gamma_{\text {rot }} / \mathrm{cm}^{-1}$ & rel. abs. & $\Delta \tilde{\nu}_{\text {abs }} / \mathrm{cm}^{-1}$ & $\Gamma_{\text {rot }} / \mathrm{cm}^{-1}$ & rel. abs. \\
\hline $16335.0 \pm 0.9$ & $18.8 \pm 1.0$ & 0.36 & $-0.9 \pm 1.0$ & $12.2 \pm 0.4$ & 0.36 \\
$16343.1 \pm 2.2$ & $18.1 \pm 4.8$ & 0.25 & $-5.4 \pm 2.3$ & $11.8 \pm 2.5$ & 0.17 \\
$16363.4 \pm 0.4$ & $14.0 \pm 1.3$ & 0.18 & $-2.0 \pm 0.5$ & $12.1 \pm 0.7$ & 0.15 \\
$16460.6 \pm 0.5$ & $19.5 \pm 1.9$ & 0.22 & $+0.5 \pm 0.6$ & $23.6 \pm 0.7$ & 0.23 \\
$16498.8 \pm 0.3$ & $35.9 \pm 1.3$ & 0.36 & $-0.5 \pm 0.4$ & $23.1 \pm 0.5$ & 0.33 \\
$16533.1 \pm 0.2$ & $22.2 \pm 0.8$ & 0.37 & $+0.2 \pm 0.3$ & $19.1 \pm 0.3$ & 0.37 \\
\hline
\end{tabular}

Column 1: $\nu_{\text {abs }}$ : absolute wavenumber; 2 and 5: approximated FWHM of rotational contour, $\Gamma_{\text {rot }}$, determined with the IBBCEA and CRD method, respectively; 3 and 6 : relative absorbance normalized to the first line determined with IBBCEAS and CRD method, respectively; 4: difference of absolute wavenumbers determined with IBBCEA and CRD method. 
way. The corresponding spectral positions $\left(\tilde{\nu}_{\text {abs }}\right)$ for the IBBCEAS measurement, position differences $\left(\Delta \tilde{\nu}_{\text {abs }}\right)$ to the CRDS data, the FWHM $\left(\Gamma_{\text {rot }}\right)$ and the relative absorption intensities (rel. abs.) are listed in Table 6.1. The given errors were calculated by the fit of the profiles only. The difference of $\Gamma_{\text {rot }}$ of the rotational contours in the two spectra is due to: (i) the lower spectral monochromator resolution of about $\approx 4 \mathrm{~cm}^{-1}$ (at $\left.605 \mathrm{~nm}\right)$ in the IBBCEAS setup compared to the laser resolution of $\approx 0.16 \mathrm{~cm}^{-1}$ in the CRD measurement and (ii) the slightly worse rotational cooling rate in the continuous jet experiments as opposed to the pulsed supersonic expansion in the CRD experiment. Hence, rotational states at higher energies are populated in the IBBCEAS experiment leading to an additional broadening of absorption features. Considering the spectral resolution of the monochromator used, the line positions of the IBBCEA measurement are in good agreement with the CRD experiment.

In Ruth99] an increase of line widths $\Gamma_{\text {rot }}$ above $16400 \mathrm{~cm}^{-1}$ was reported and interpreted as experimental evidence for a conical intersection of the $\mathrm{S}_{0}$ and $\mathrm{S}_{1}$ potential energy surfaces $\sim 2100 \mathrm{~cm}^{-1}$ above the $S_{1,0}$ origin. This increase of line widths is also evident in the IBBCEA spectrum in Fig. 6.4 despite the lower resolution of the method (see also Table 6.1). The very good agreement of these results shows, that the IBBCEA technique is just as applicable for spectroscopy in supersonic jets as the CRD absorption method.

\subsection{Variation of Experimental Parameters}

To optimize the jet conditions with respect to the molecule temperature and the absorption signal the absorption was measured in the region between 16260 and $16611 \mathrm{~cm}^{-1}$ by varying: (i) the position of the nozzle, (ii) the temperature of the oven and the reservoir and (iii) the argon stagnation pressure. 


\section{(i) Position of the nozzle:}

The nozzle and the oven were mounted together on translational stages which could move them in three different directions. The pipe for the carrier gas, which was connected to the oven at one side and to a feed-through in the vacuum chamber on the other side, was coiled to a spiral and therefore flexible even though it was made from aluminium. Due to the large angle of supersonic beam spread $\left(\sim 90^{\circ}\right)$ and the large diameter $(25 \mathrm{~mm})$ of the cavity used for the IBBCEA measurements, displacing the nozzle by even $\sim 5 \mathrm{~mm}$ perpendicular to the jet direction had little effect to the absorption signal. Yet translations of the nozzle parallel to the axis of the jet had a serious influence to the absorption signal: At larger distances between the optical axis of the cavity and the nozzle the absorption dropped dramatically because of the decreasing number density of azulene molecules in the probe volume of the cavity. Close distances were limited by the radius of the probe volume $(12.5 \mathrm{~mm})$. The nozzle had to be just outside of this volume, otherwise it would partially cover the optical path and add to the measured absorption loss. Therefore a distance of $13 \mathrm{~mm}$ was kept for all IBBCEA measurements presented later in this section.

At this point it should be noted that only absorptions of an averaged molecule temperature is measured due to the large probe volume. The probe volume includes distances from the nozzle between 0 and $25 \mathrm{~mm}$, in which the molecules may have different temperatures. The distortion of the absorption lines however is small as can be seen in Fig. 6.4. The shown CRD measurement has only one temperature $\left(T_{\text {trans }} \sim 6 \mathrm{~K}, T_{\text {rot }}=(9 \pm 2) \mathrm{K}\right)$ since the probe diameter, which is effectively defined by the beam diameter $(\sim 1 \mathrm{~mm})$, is small versus the distance to the nozzle of $8 \mathrm{~mm}$.

(ii) Temperature of the oven:

The temperature of the oven had a significant influence to the absorption signal 
since the vapour pressure depends strongly on the temperature. With increasing temperature the number of absorbing azulene molecules in the vapour phase increases, but the material consumption also increases the probability of cluster formation together with the absorption background by vestiges of hot azulene in the rest gas. As another condition, which in the case of azulene was irrelevant, the molecule must not be thermally destroyed. The optimum temperature range for seeding azulene into the jet was established to be between 95 and $125^{\circ} \mathrm{C}$, where the influence of the absorption background could be kept below $10 \%$ of the absorption signal. The ratio of seeded azulene molecules to the Ar carrier gas atoms was smaller than $1: 100$ for these temperatures and azulene complex formation were therefore negligible. Also the observation of Ar-azulene complexes in the spectrum is negligible owing to the unfavourable expansion conditions for cluster formation (i.e., high temperature and low argon stagnation pressure).

\section{(iii) Argon stagnation pressure:}

According to the supersonic jet literature a high argon stagnation pressure should lead to a better cooling of the seeded molecule in the jet due to the increased number of collisions with the carrier gas atoms. Thus less rovibrational and roational states are excited and more molecules are in the ground state. Narrower and therefore more intense absorption features should be the effect. But a variation of the pressure showed that at the lowest stable pressure of the jet system (300 mbar), the strongest absorption signal was obtained. This behaviour was attributed to the fact that different flow rates of the carrier gas (e.g., $\sim 10 \mathrm{~cm}^{3} / \mathrm{s}$ at $1000 \mathrm{mbar})$ cool the oven volume $\left(\approx 35 \mathrm{~cm}^{3}\right)$ differently. Hence, the absorption signal decreased according to the lower number density of azulene in the molecular beam at higher flow velocities. This effect is demonstrated in Fig. 6.5 where four spectra at different argon stagnation pressures are shown. For all measurements shown in the figure, the quartz oven was kept at a temperature of $117 \pm 3^{\circ} \mathrm{C}$ and 


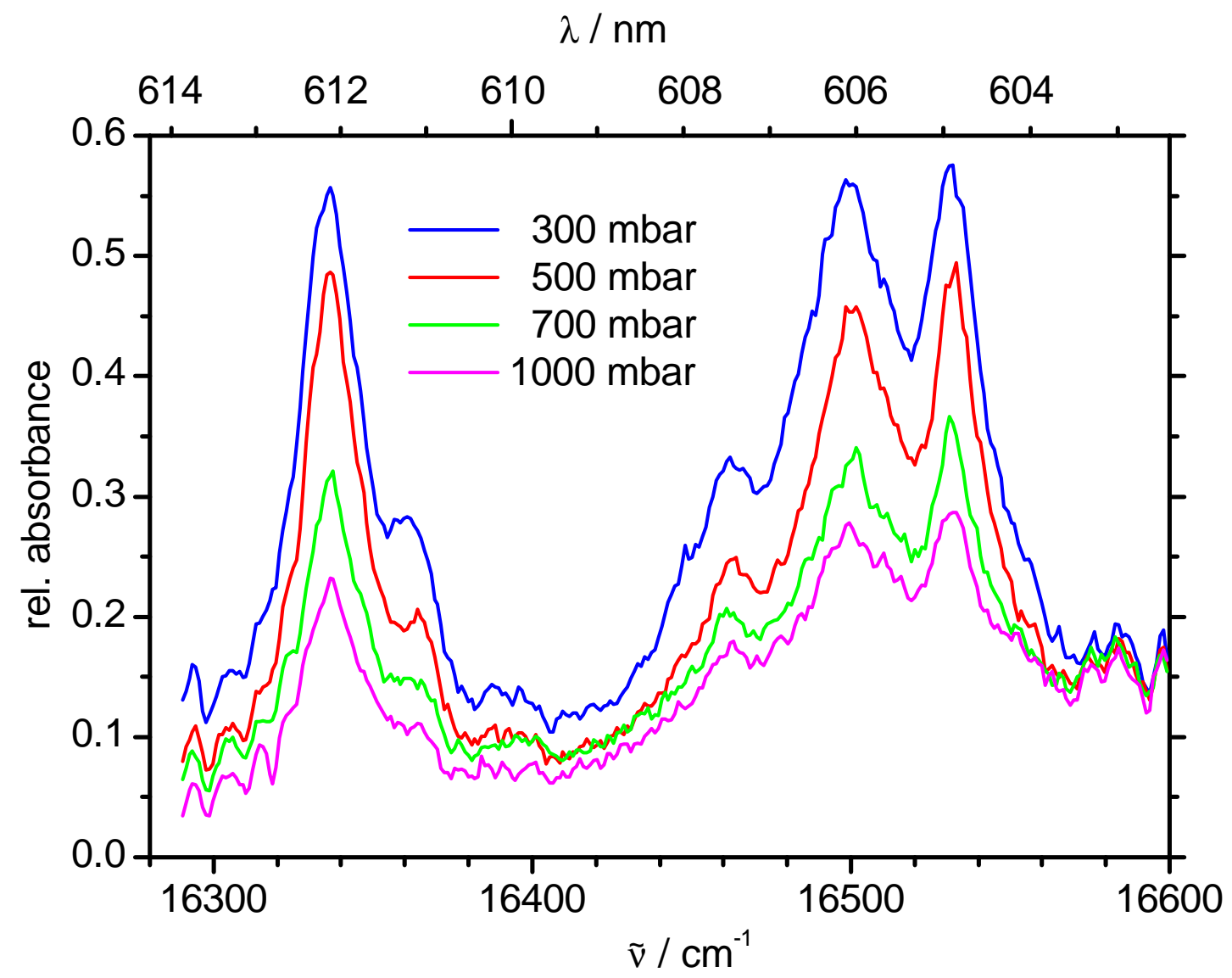

Figure 6.5: IBBCEAS experiment with jet-cooled azulene in the same vibronic region as in Fig. 6.4 measured for the same oven temperature $\left(117 \pm 3^{\circ} \mathrm{C}\right)$ at four different argon stagnation pressures.

the exposure time for the IBBCEAS measurement was $100 \mathrm{~s}$. Within the limits of the spectral resolution the rotational cooling rate did not seem to be affected, since the widths of the contour bands remained the same in all four measurements.

The broad-band observation of absorption spectra by IBBCEAS allows - as demonstrated above - the optimization of several experimental parameters of the supersonic jet. This simplified the measurement procedure and reduced, compared to a CRD setup, the time needed to obtain the spectra presented below. 


\subsection{Results of IBBCEAS of Azulene in a Supersonic Jet}

At an argon pressure of 300 mbar the $\mathrm{S}_{1} \leftarrow \mathrm{S}_{0}$ absorption of jet-cooled azulene was measured with IBBCEAS between 17059 and $20255 \mathrm{~cm}^{-1}$, a region which was not addressed in Ruth99. Fig. 6.6 shows this spectral range together with the low energy part of the $\mathrm{S}_{1} \leftarrow \mathrm{S}_{0}$ absorption spectrum $\left(14235-17300 \mathrm{~cm}^{-1}\right)$ obtained with CRDS [Ruth99] and a simulated spectrum which is discussed in section 6.7. The IBBCEA spectrum was assembled from eight separate absorption measurements in overlapping spectral regions, each covering $\approx 14 \mathrm{~nm}\left(\approx 400 \mathrm{~cm}^{-1}\right)$; the exposure time per individual spectrum varied according to the signal between 300 and $1200 \mathrm{~s}$. The oven temperature was gradually increased from $95^{\circ} \mathrm{C}$ at $17100 \mathrm{~cm}^{-1}$ to $125^{\circ} \mathrm{C}$ at $20255 \mathrm{~cm}^{-1}$, due to worse mirror reflectivities and lower absorbance of azulene at higher excess energies. The CRDS experiment used a pulsed expansion through a nozzle $€^{\mathrm{a}}$ orifice of $1 \mathrm{~mm}$. The stagnation pressure was 1000 mbar helium and the reservoir temperature was held at $90^{\circ} \mathrm{C}$ all the time.

The IBBCEA spectrum was adjusted to the local signal of the CRD absorption spectrum of [Ruth99] in the overlapping region between 17100 and $17300 \mathrm{~cm}^{-1}$. One of the known benefits of cavity ring-down spectroscopy is that it is insensitive with respect to intensity fluctuations of the light source. At first glance, this advantage seems to be lost if the cavities' transmission is measured instead of the ring-down time in IBBCEAS. However, using a white-light source allows averaging the intensity within the entire spectral region of high mirror reflectivity at one time. Hence, intensity fluctuations of the lamp do not add to the noise, however may result in a shift of the baseline. Therefore, and since somewhat

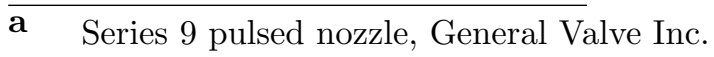



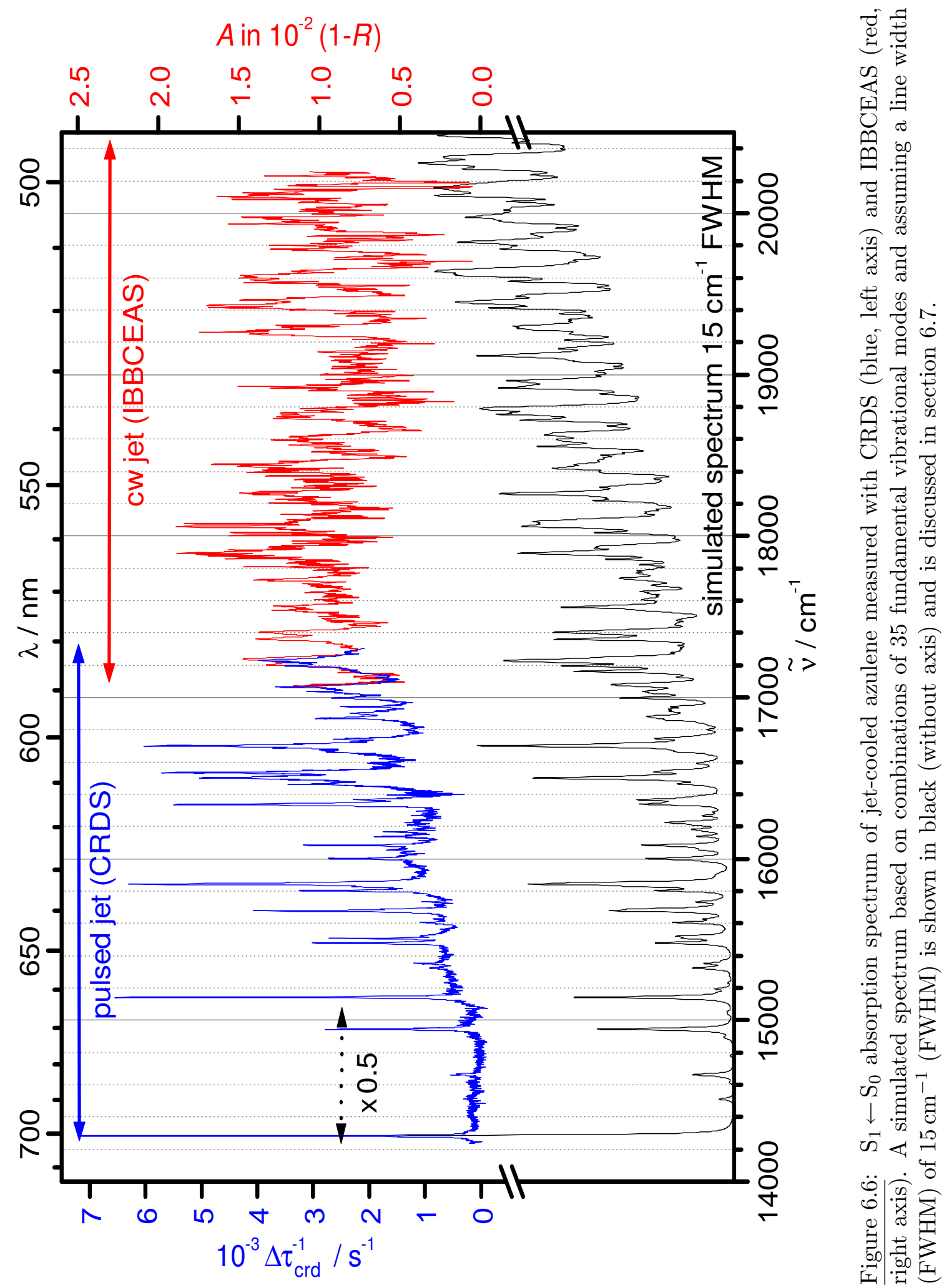
different mirror reflectivities and small variations of the expansion temperature for measurement in different spectral regions also influence the absorption signal, the shape of the absorption background above $17300 \mathrm{~cm}^{-1}$ does not represent the real situation. Due to these practical limitations the relative absorptions are not reliable in terms of the background absorption, although all spectral regions are based on a normalization to the known CRD absorption spectrum. Consequently, the interesting problem whether the absorption background at high excess energies contains a significant absorption contribution of extremely short-lived $\mathrm{S}_{1, \nu}$ states above a conical intersection $\left(\tau\left(\mathrm{S}_{1, \nu}\right) \ll 1\right.$ ps above $\left.16400 \mathrm{~cm}^{-1}\right)$, or whether the background is simply a consequence of an increased congestion of the spectrum in combination with the incomplete rotational cooling of azulene molecules in the jet, can unfortunately not be reliably addressed on basis of the presented data.

However, IBBCEAS is sufficient to monitor the spectral structure in the high excess energy region, despite its somewhat lower resolution in comparison to pulsed CRDS. At excess energies $>17300 \mathrm{~cm}^{-1}$ the spectrum is dominated by recurring, broad absorption features which could not be assigned to individual vibronic $\mathrm{S}_{1, \nu} \leftarrow \mathrm{S}_{0,0}$ transitions. The periodic nature of the absorption bands suggests that the observed spectrum is dominated by only a small number of progressions. Hot bands in the spectrum resulting from the population of the lowest energy vibration in azulene at $\approx 228 \mathrm{~cm}^{-1}$ (see Ruth99]) cannot be entirely ruled out. However, hot azulene molecules cannot possibly contribute more than $\sim 3.8 \%$ to the relative absorbance $A$, if a vibrational temperature of $100 \mathrm{~K}$ is assumed as well as Franck-Condon factors for excitation of hot azulene being the same or smaller than for the ground vibrational state. The observation of Ar-azulene complexes in the spectrum is negligible owing to the unfavourable expansion conditions for cluster formation (i.e., high temperature and low argon stagnation pressure). 


\subsection{Calculation of the Azulene Spectrum}

The $\mathrm{S}_{1, \nu} \leftarrow \mathrm{S}_{0,0}$ azulene spectrum consists of absorption lines due to transitions from the electronic ground state $\mathrm{S}_{0}$ to vibronically excited states in $\mathrm{S}_{1}$. Azulene possesses 17 totally symmetric $a_{1}$ modes, 16 in-plane modes of $b_{1}$ symmetry as well as $9 \mathrm{~b}_{2}$ and $6 \mathrm{a}_{2}$ out-of-plane modes Negri93. Each possible combination of an arbitrary number of fundamental modes potentially contributes to the density of eigenstates Haarhoff63] in the singlet manifold and hence to the line density in the observed spectrum. For a comprehensive overview of the involved quantum mechanics confer for instance Ref. [Demtröder03].

In a simple but reasonable model, the absorption of combination modes can be calculated by the product of the absorption strength of the measured corresponding single modes forming the contour band. According to the Born-Oppenheimer approximation, the electronic, vibrational and rotational parts of the wave function describing the molecule can be separated. Considering only different vibronic transitions between two selected electronic states, the probability of a transition is proportional to the Franck-Condon factor

$$
f_{\mathrm{A} \rightarrow \mathrm{B}}=\left|\int \psi_{\mathrm{A}}(\mathbf{r}) \times \psi_{\mathrm{B}}(\mathbf{r}) d \mathbf{r}\right|^{2} .
$$

It depends on the overlap of the vibrational wave functions $\psi_{\mathrm{A}}(\mathbf{r})$ and $\psi_{\mathrm{B}}(\mathbf{r})$ of the participating states $\mathrm{A}$ and $\mathrm{B}$, respectively. Using normal coordinates $r_{i}$ of the fundamental vibration eigenmodes $\nu_{i}$ in the upper electronic level, the vibrational wave functions can be written as the product of eigenmode wave functions:

$$
\psi_{\nu}(\mathbf{r})=\psi_{\nu_{1}}\left(r_{1}\right) \times \psi_{\nu_{2}}\left(r_{2}\right) \times \psi_{\nu_{3}}\left(r_{3}\right) \times \ldots
$$

The Franck-Condon factor $f_{\mathrm{A} \rightarrow \mathrm{B}+\mathrm{C}}$ for a transition from a ground state $\mathrm{A}$ in the 
lower electronic level to a state of combined vibrations B and C (different from B) in the upper electronic state can then be calculated:

$$
\begin{aligned}
f_{\mathrm{A} \rightarrow \mathrm{B}+\mathrm{C}} & =\left|\prod_{i=1}^{n} \int \psi_{\mathrm{A}_{i}}\left(r_{i}\right) \times \psi_{(\mathrm{B}+\mathrm{C})_{i}}\left(r_{i}\right) d r_{i}\right|^{2} \\
& =\left|\prod_{i=1}^{n} \int \psi_{\mathrm{A}_{i}}\left(r_{i}\right) \times \psi_{\mathrm{B}_{i}}\left(r_{i}\right) d r_{i} \times \int \psi_{\mathrm{A}_{i}}\left(r_{i}\right) \times \psi_{\mathrm{C}_{i}}\left(r_{i}\right) d r_{i}\right|^{2} \\
& =f_{\mathrm{A} \rightarrow \mathrm{B}} \times f_{\mathrm{A} \rightarrow \mathrm{C}}
\end{aligned}
$$

Consequently, if the Franck-Condon factors $f_{\mathrm{A} \rightarrow \mathrm{B}}$ and $f_{\mathrm{A} \rightarrow \mathrm{C}}$ are known for two transitions $\mathrm{S}_{1, \mathrm{~B}} \leftarrow \mathrm{S}_{0, \mathrm{~A}}$ and $\mathrm{S}_{1, \mathrm{C}} \leftarrow \mathrm{S}_{0, \mathrm{~A}}$, the Franck-Condon factor $f_{\mathrm{A} \rightarrow \mathrm{B}+\mathrm{C}}$ for the transition $\mathrm{S}_{1, \mathrm{~B}+\mathrm{C}} \leftarrow \mathrm{S}_{0, \mathrm{~A}}$ can be calculated by the multiplication of $f_{\mathrm{A} \rightarrow \mathrm{B}}$ and $f_{\mathrm{A} \rightarrow \mathrm{C}}$. Since the measured absorption strengths of the lines contain the information about the associated Franck-Condon factors, the product of the absorption strengths normalized to the $\mathrm{S}_{1,0} \leftarrow \mathrm{S}_{0,0}$ transition results in the relative transition strength of the combined vibration modes.

However, it is important to note that for the presented approach selection rules due to the symmetry of the wave functions, vibronic anharmonicities as well as "interactions" between close energy levels (e.g. Fermi resonances) were not considered. The wave functions of overtones were assumed to have the same symmetry and spatial distribution as their fundamental modes since some observed overtone lines show absorption strengths in the same order of magnitude. For an accurate calculation of a spectrum the line strengths and positions of all fundamental modes involved have to be known.

In a previous work, several fundamental modes in the $\mathrm{S}_{1, \nu}$ state of azulene could be assigned to spectral line positions. A table of absorption features presented and assigned in [Ruth99] can be found in appendix A.3. The fundamental modes 
used to calculate the simulated spectrum are marked. A fast algorithm to directly compute harmonic oscillator eigenstate densities [Stein72] was modified to take the absorption strength into account. The absorption strength for each vibrational combination was calculated as outlined above and added up for each line position. In a second step Lorentzian profiles were calculated for each line and added up to the overall absorption spectrum. The full widths at half maximum (FWHM) for the Lorentzian profiles used are stated in the figures.

Considering the simplicity of this approach to simulate the spectrum, the agreement between the calculated and measured spectrum in Fig. 6.6 is remarkable. The majority of the absorption features were reproduced for excess energies smaller than $3000 \mathrm{~cm}^{-1}$. Most of the broad absorption structures observed in the IBBCEA spectrum at high excess energies are also reproduced with an absorption strength of the right order of magnitude. The discrepancy in the positions of the maxima around $20000 \mathrm{~cm}^{-1}$ could on one hand be caused by anharmonicities of the vibronic potential, on the other hand small uncertainties of the measured line positions for the fundamental modes may add up to cause relatively large deviations of the calculated energies for high order combinations.

A few measured absorption bands were not reproduced in the calculation. This could be an indication that a few fundamental modes were wrongly assigned to combination modes in [Ruth99] or that symmetry selection rules prohibit the transition to e.g. $\mathrm{a}_{2}$ fundamental modes but not $\mathrm{a}_{2}$ combination modes of same. Since the line width was assumed to be constant for all lines of the calculated spectrum, this observation could also be caused by variations of the line widths in the measured spectrum: Measured line widths smaller than assumed for the calculation would raise the amplitude of the absorption line in comparison to the calculated spectrum while larger line widths would reduce it.

Even the background, which is increasing with the energy above $15000 \mathrm{~cm}^{-1}$, 
was calculated quite well. The energy, at which the background begins to raise in the simulation, depends strongly on the line width used for the Lorentzian profiles. Since the maximum absorption strengths and not the wavelength integrated absorption were used for the simulation, an alteration of the line width leads to a change of the overall absorption. The absorption strengths of 7 very weak - Herzberg-Teller allowed but Franck-Condon inhibited - transitions below $1000 \mathrm{~cm}^{-1}$ were reported in Ruth99 to be close to the noise of the measurement and could not be determined accurately. For the simulations presented in this work they were set to $1 \%$ of the band origin. However, simulations using different values $(0 \%-2 \%)$ for these lines showed that the background depends also on their absorption strengths due to the high order of combinations possible in the considered energy range.

Using 35 fundamental modes as listed in Appendix A.3 for the calculation, the simulated spectrum consists of $\sim 22 \times 10^{6}$ combination modes in the region up to $6300 \mathrm{~cm}^{-1}$ above the band origin. A line density of 30 lines per $\mathrm{cm}^{-1}$ is reached at an excess energy of $\sim 3000 \mathrm{~cm}^{-1}$, it increases almost exponentially at higher excess energies above $S_{1,0}$. At $\sim 6000 \mathrm{~cm}^{-1}$ the value of 10000 lines per $\mathrm{cm}^{-1}$ is exceeded.

\subsection{Discussion}

In [Ruth99] and [Wurzer99] evidence for a conical intersection of the $\mathrm{S}_{0}$ and $\mathrm{S}_{1}$ potentials of azulene was reported. This conclusion was founded on a change of the line widths from 10 to $20 \mathrm{~cm}^{-1}$ at $\approx 2100 \pm 100 \mathrm{~cm}^{-1}$. Above this critical excess energy the lifetime of the state is reduced due to a fast relaxation channel via a conical intersection. The absorption spectrum above this region is shown in Fig. 6.7 as a function of the absolute wavenumber, $\tilde{\nu}_{\text {abs }}$, or the excess energy $\tilde{\nu}_{\text {rel }}$ 


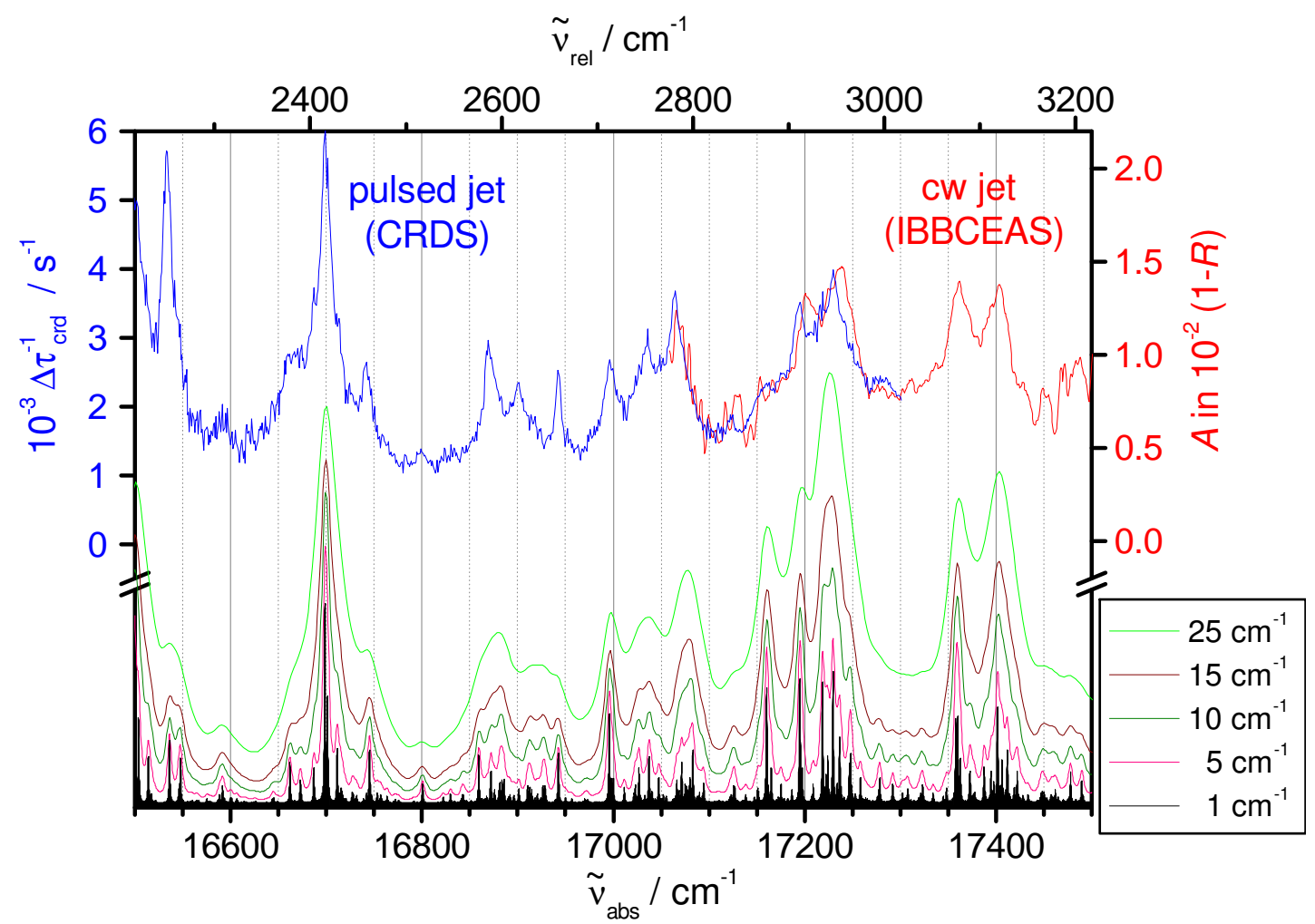

Figure 6.7: Part of the $\mathrm{S}_{1} \leftarrow \mathrm{S}_{0}$ absorption spectrum of jet-cooled azulene measured with CRDS (blue, left axis) and IBBCEAS (red, right axis). For the calculated spectrum line widths (FWHM) of 1, 5, 10, 15 and $25 \mathrm{~cm}^{-1}$, respectively, were used in the computation shown in the bottom of the graph. The axis for the calculated spectra is the same for all line widths but is not shown here.

relative to the band origin, respectively. A stick spectrum at the bottom of the graph (black, FWHM $1 \mathrm{~cm}^{-1}$ ) shows that all absorption structures are composed of several very close lines. However, the comparison between the measured spectrum and the simulated spectra shows in accordance with findings in [Ruth99] that broad line widths between 15 and $25 \mathrm{~cm}^{-1}$ dominate the spectrum (e.g. around $17400 \mathrm{~cm}^{-1}$ ), while still some narrow absorption features (e.g. $16870 \mathrm{~cm}^{-1}$ ) exist.

According to Olivucci and co-workers [Olivucci99], above the conical intersection a so-called impulsive decay channel is opened, where part of the molecules decay via a direct and totally symmetric coordinate, involving the contraction of 


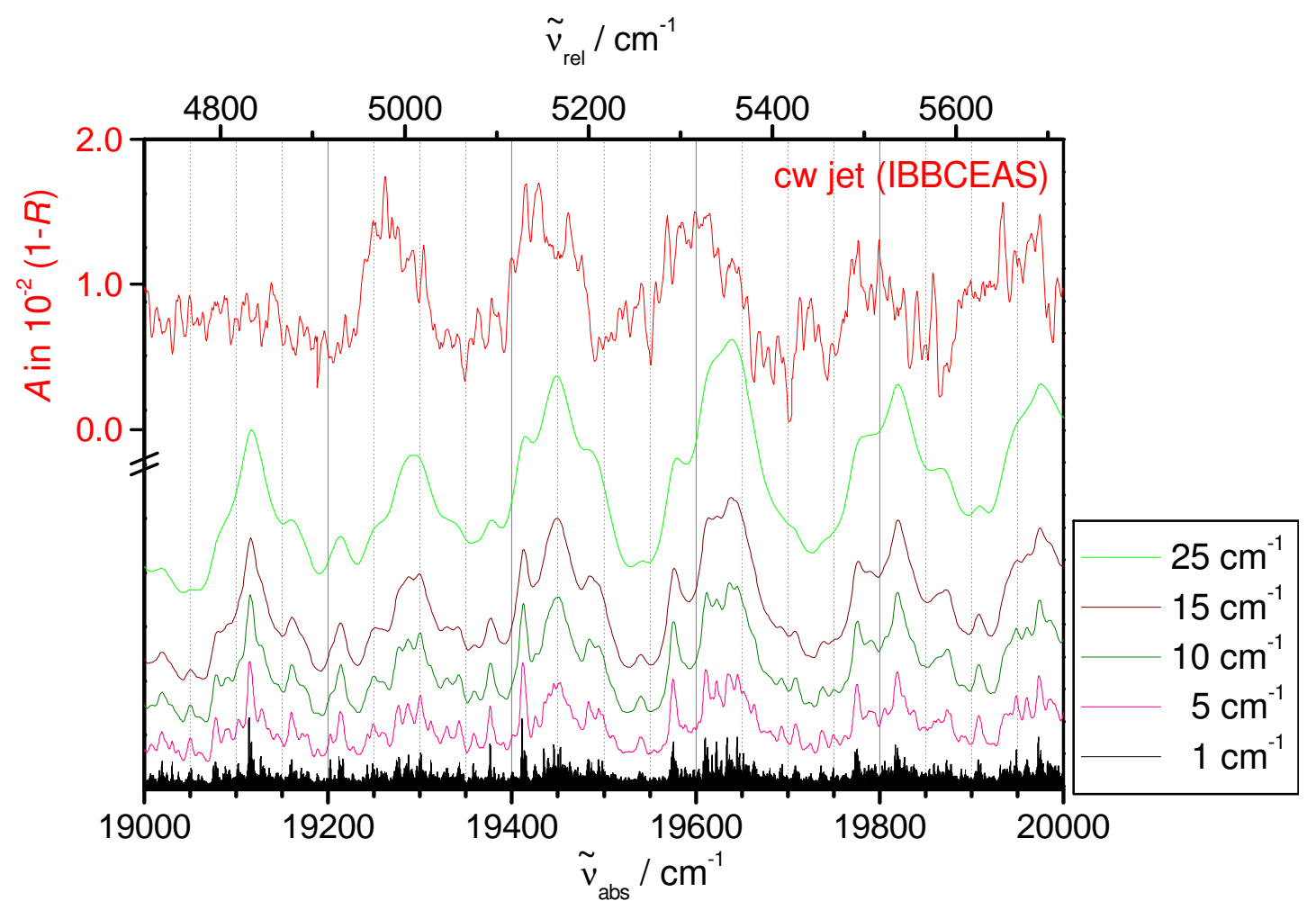

Figure 6.8: Part of the $\mathrm{S}_{1} \leftarrow \mathrm{S}_{0}$ absorption spectrum of jet-cooled azulene measured with IBBCEAS (red, left axis). For the calculated spectrum line widths (FWHM) of 1, $5,10,15$ and $25 \mathrm{~cm}^{-1}$, respectively, were used in the computation, shown in the bottom of the graph. The axis for the calculated spectra is the same for all line widths but is not shown here.

the transannular bond and/or the single-bond double-bond alternation mode in azulene, whereas others "orbit" the intersection on coordinates away from the $\mathrm{C}_{2 \mathrm{v}}$ symmetry. This picture accounts for the somewhat random behavior of the linewidths in Fig. 6.7. The variation in linewidths can be explained such that, after excitation to the Franck-Condon point no reaction coordinate is directly connected to the conical intersection $\mathrm{S}_{1} / \mathrm{S}_{0}$.

For very high excess energies $\left(>4000 \mathrm{~cm}^{-1}\right)$ the measured spectrum exhibits a structure of very dense narrow lines. Due to the high line density it can not be decided if the existing broad absorption features (see Fig. 6.8) consist only of nar- 
row lines or if narrow lines on the back of broad lines are responsible for these structures. The narrow line structure shows line widths similar to the ones in the spectrum simulated with a FWHM of $5 \mathrm{~cm}^{-1}$ (e.g. at $19570 \mathrm{~cm}^{-1}$ ). However, the resolution of the setup was only $\sim 4 \mathrm{~cm}^{-1}$ and therefore the real line widths could even be less. The fact that such a rich vibronic structure is observed even at excess energies approaching $6000 \mathrm{~cm}^{-1}$ is quite surprising and an important result, since consequently vibronic $\mathrm{S}_{1, \nu}$ states with lifetimes of several hundred femtoseconds must still exist well above the conical intersection. The corresponding reasonably strong transitions lead to excitation of Franck-Condon states with an unfavourable reaction coordinate regarding internal conversion involving relaxation via the conical intersection.

The half widths of the broader absorption features $\left(50-100 \mathrm{~cm}^{-1}\right)$ are similar to the half widths of the broad structures of the CRD spectrum at energies $>16400 \mathrm{~cm}^{-1}$. In the calculated spectra such broad structures only occur for line widths $\geq 10 \mathrm{~cm}^{-1}$. Due to the high line density, the simulated spectrum shows an absorption background which increases with the excess energy for all line widths $\geq 5 \mathrm{~cm}^{-1}$. This agrees with the background observed in the CRDS experiments, a further increasing background however could not be verified experimentally using IBBCEAS (see section 6.6). In this context, it is important to note that for high line densities a background due to overlapping lines of small half widths cannot be distinguished from overlapping lines of large half widths. Therefore it is not possible to rule out or to confirm contribution to the absorption by extremely short-lived states ( $50 \mathrm{fs} \approx 100 \mathrm{~cm}^{-1}$ ) which decay directly via the conical intersection as predicted in Olivucci99. 


\subsection{Summary}

The application of incoherent broad-band cavity-enhanced absorption spectroscopy to isolated jet-cooled gas phase species was demonstrated on basis of the $\mathrm{S}_{1} \leftarrow \mathrm{S}_{0}$ transition of azulene in a continuous supersonic expansion. The absorption was measured over a broad spectral range between 17300 and $20255 \mathrm{~cm}^{-1}$ with a resolution of $\approx 4 \mathrm{~cm}^{-1}$. The resolution, which is due to the dispersion of transmitted light by a grating monochromator, was sufficient to monitor the highly congested vibronic structure $>3000 \mathrm{~cm}^{-1}$ above the $\mathrm{S}_{1,0}$ origin of azulene. The sensitivity required for this absorption experiment compares well to that of conventional pulsed cavity ring-down measurements.

Even though individual assignments of vibrational mode combinations had to be abandoned, a simple model was presented to calculate a spectrum, based on identified fundamental vibronic transitions. The simulated spectrum, reproduced the main absorption structures in the measured spectrum for certain homogeneous line widths used. The increasing absorption background is reproduced qualitatively. However, due to the high line density it was not possible to rule out or confirm a contribution of extremely short-lived states to the absorption $\left(50 \mathrm{fs} \approx 100 \mathrm{~cm}^{-1}\right)$ which decay directly via the conical intersection. 


\section{Chapter 7}

\section{Prospects of the IBBCEA technique}

\section{Issues to be addressed}

Incoherent broad-band cavity-enhanced absorption spectroscopy proved to be a cheap, powerful and very useful tool for absorption spectroscopy. However, there are several, in this context particularly important issues to be addressed:

(i) How can the mirror reflectivities $R$ be determined, using a IBBCEA setup?

Since the knowledge of the reflectivity is required to determine the absorption coefficient, a simple calibration measurement of a known sample is necessary to calculate the reflectivity. Absorption lines of small molecules however are inappropriate, since no absorption can be observed between the lines and hence no mirror reflectivities can be calculated there. Probably more suitable are the scattering properties of molecules, since the optical scattering losses cover the whole visible 
spectrum and simple molecules like $\mathrm{N}_{2}$ could be used for which the scattering cross sections are well known [Naus00].

(ii) What is the optimal geometry to inject the maximum light intensity into the cavity and from there into the spectrometer for the IBBCEA setup?

The geometrical theory of optical imaging for lenses and mirrors and their aberrations, the theory of diffraction at apertures as well as the theory of interferences in an optical cavity are well understood in optics [Born80]. The propagation of light along the optical path of an IBBCEA setup can be calculated using Gaussian optics or these days by ray tracing programs, but both require some computational effort.

\section{Future Applications}

Incoherent broad-band cavity-enhanced absorption spectroscopy (IBBCEAS) proved to have the same sensitivity potential like cavity ring down spectroscopy (CRDS). Therefore all fields of application of CRDS, where the high resolution of a laser is unnecessary, are transferable to IBBCEAS.

A good example is the growth of thin solid layers, which is of high interest for semiconductor industries and modern solid state physics. Due to the low absorption of thin samples a highly sensitive technique is required to monitor the thickness of the layer spectrally [Pipino00, Engeln99.

However, in principle a measurement of a transparent solid substrate in an IBBCEA setup was already presented in section 4.1 and it was shown that the reflection losses of the substrate in the cavity are small as long as the surfaces are perpendicular or in Brewster arrangement to the optical axis. Absorptions induced by 
molecules placed in or on the substrate should be detectable. In fact, in a preliminary experiment it was tried to deposit a layer of biphenylene molecules on the mirror surfaces by vaporising them in an evacuated cavity cell. However, only a strong but wavelength independent optical loss was observed, probably due to scattering at biphenylene crystals on the mirror surfaces.

There are also several modified IBBCEA setups conceivable, which may have diverse advantages:

Pulsed light sources: Although IBBCEAS was presented here as a cw technique, a pulsed application (e.g. pulsed supersonic jets) is possible if a convenient pulsed light source or pulsed detector is used. However one should bear in mind that the sensitivity depends on the number of photons detected and flash lamps or pulsed LED's should be preferred to chopping light.

Ultra bright light emitting diodes (LED's) as light sources are very cheap, have favourable imaging properties owing to the small light spots $(\sim 200 \mu \mathrm{m})$, have a relatively large spectral luminance and supersede optical bandpass filters, both due to the narrow light spectrum emitted ( $20 \mathrm{~nm}$ FWHM). Quite recently a broad-band cavity-enhanced absorption experiment using light emitting diodes was reported [Ball04], achieving a sensitivity of $5.2 \times$ $10^{-9} \mathrm{~cm}^{-1}$ with a resolution of $0.09 \mathrm{~nm}$ FWHM over a range of $\Delta \lambda=38 \mathrm{~nm}$.

Echelle spectrometers are able to measure simultaneously a spectral region of $\sim 100 \mathrm{~nm}$ with high resolution $(\sim 0.01 \mathrm{~nm})$.

Fourier transform spectrometers do not require focussing through very small slits but on the detector area only $\left(\sim 1 \mathrm{~cm}^{2}\right)$. But due to the very high achievable resolution of $\sim 0.001 \mathrm{~cm}^{-1}(\hat{=} 0.000025 \mathrm{~nm}$ at $500 \mathrm{~nm})$, mode problems could occur. A FT-spectrometer is a scanning technique, not in the 
frequency domain but in the interferometric length, and therefore susceptible to fluctuations of intensity and density of the sample

Without spectrometers: Some traditional analytical methods (e.g. atomic absorption spectroscopy) do not depend on wavelength dispersion. If the light source used provides only light at the same wavelengths as the absorption of the sample (e.g. atomic lines) no spectrometer is necessary. A cavity could increase the effective path length and therefore enhance the sensitivity.

Considering the rapid development of laser based cavity-enhanced spectroscopy in the last years and the extensive commercial application of classical spectroscopy techniques, reports of diverse new designs and still higher sensitivity limits may be expected in the near future.

a First FT-CEA spectra were presented quite recently on the CRD-user meeting 2004 by R. Zijlmans (of the research group around R. Engeln, Eindhoven University, Netherlands). 


\section{Chapter 8}

\section{Conclusion}

Incoherent broad-band cavity-enhanced absorption spectroscopy (IBBCEAS) combines the simplicity and robustness of standard non-invasive optical absorption spectroscopy with the enhancement concepts developed in cavity ring-down spectroscopy. In this work the basic equations to describe the IBBCEA technique were derived and verified in proof-of-principle experiments for liquid and gaseous samples as well as for measurements in a supersonic jet.

Using an optical cavity with mirror reflectivities of $R=0.99$ for instance in a commercial absorption spectrometer for the measurement of liquid samples in standard cuvettes resulted in an improved signal-to-noise ratio which agreed with the expected enhancement factor of $Q=7$. By measuring the weak $\mathrm{b}^{1} \Sigma_{g}^{+}\left(v^{\prime}=2\right) \leftarrow$ $\mathrm{X}^{3} \Sigma_{g}^{-}\left(v^{\prime \prime}=0\right)$ triplet-singlet transition of molecular oxygen with mirrors of $R=$ 0.9999 and a spectrometer resolution of $0.66 \mathrm{~cm}^{-1}(\approx 0.026 \mathrm{~nm})$, a sensitivity of $3 \times 10^{-8} \mathrm{~cm}^{-1}$ for the absorption coefficient was demonstrated.

Using IBBCEAS, first spectra were recorded as a function of time of the recently discovered complex oscillatory absorption behaviour induced by UV multi-photon photolysis in a closed gas-phase system of naphthalene in a noble gas. A com- 
parison to known spectra of molecules, suspected to be involved in the oscillation process, showed that the carrier of the oscillating absorption is neither naphthalene $\left(\mathrm{C}_{10} \mathrm{H}_{8}\right)$, nor naphthalene cation $\left(\mathrm{C}_{10} \mathrm{H}_{8}{ }^{+}\right)$, nor azulene $\left(\mathrm{C}_{10} \mathrm{H}_{8}\right)$, nor benzene $\left(\mathrm{C}_{6} \mathrm{H}_{6}\right)$ or its cation $\left(\mathrm{C}_{6} \mathrm{H}_{6}{ }^{+}\right)$. The measured absorption features are very smooth and broad $(\sim 30 \mathrm{~nm})$. The possibility of condensation and rooting was investigated by considering different wavelength dependent scattering backgrounds in the fit procedure, but the spectral region covered $(630-715 \mathrm{~nm})$ was too small to determine unambiguously if scattering plays a role in the measured oscillation of the absorption. A very small temporal delay $(\sim 1 \mathrm{~s})$ between absorption features $(655 \mathrm{~nm}$ and $695 \mathrm{~nm})$ was interpreted as evidence for at least two (yet unidentified) different species involved in the oscillation process. This observation and the low estimated number of molecules transformed directly by the photolysis support an autocatalytic chemical reaction scheme causing the oscillations as already supposed in Gash04].

The application of IBBCEAS to isolated jet-cooled gas phase species was demonstrated on basis of the $\mathrm{S}_{1} \leftarrow \mathrm{S}_{0}$ transition of azulene in a continuous supersonic expansion. The absorption was measured over a broad spectral range between 17300 and $20255 \mathrm{~cm}^{-1}$ with a resolution of $\approx 4 \mathrm{~cm}^{-1}$. A highly congested vibronic structure $>3000 \mathrm{~cm}^{-1}$ above the $\mathrm{S}_{1,0}$ origin of azulene was observed. The sensitivity required for this absorption experiment compares well to that of conventional pulsed cavity ring-down measurements. Even though individual assignments of vibrational mode combinations had to be abandoned, a simple model could be found to calculate a spectrum, basing on identified fundamental vibronic transitions. The spectrum, simulated with different line widths, reproduced all the main absorption structures of the measured data. Actually even the increasing absorption background is reproduced qualitatively. However, due the high line density it was not possible to rule out or to affirm a contribution to the absorption by ex- 
tremely short-lived states $\left(50 \mathrm{fs} \approx 100 \mathrm{~cm}^{-1}\right.$ ) which decay directly via the conical intersection.

Considering the rapid development of laser based cavity-enhanced spectroscopy in the last years and the extensive commercial application of classical spectroscopy techniques, reports of diverse new designs and still higher sensitivity limits may be expected in the near future. 


\section{Appendix A}

\section{A.1 Mirror Reflectivities}

The reflectivities of all mirrors shown in this section were measured by the means of a Perkin-Elmer Lambda 7 spectrometer. The mirrors shown in Fig. A.1 were used for IBBCEAS experiments with liquids (section 4). They were produced by Layertec GmbH, Mellingen Germany, and are from the same vaporization charge but

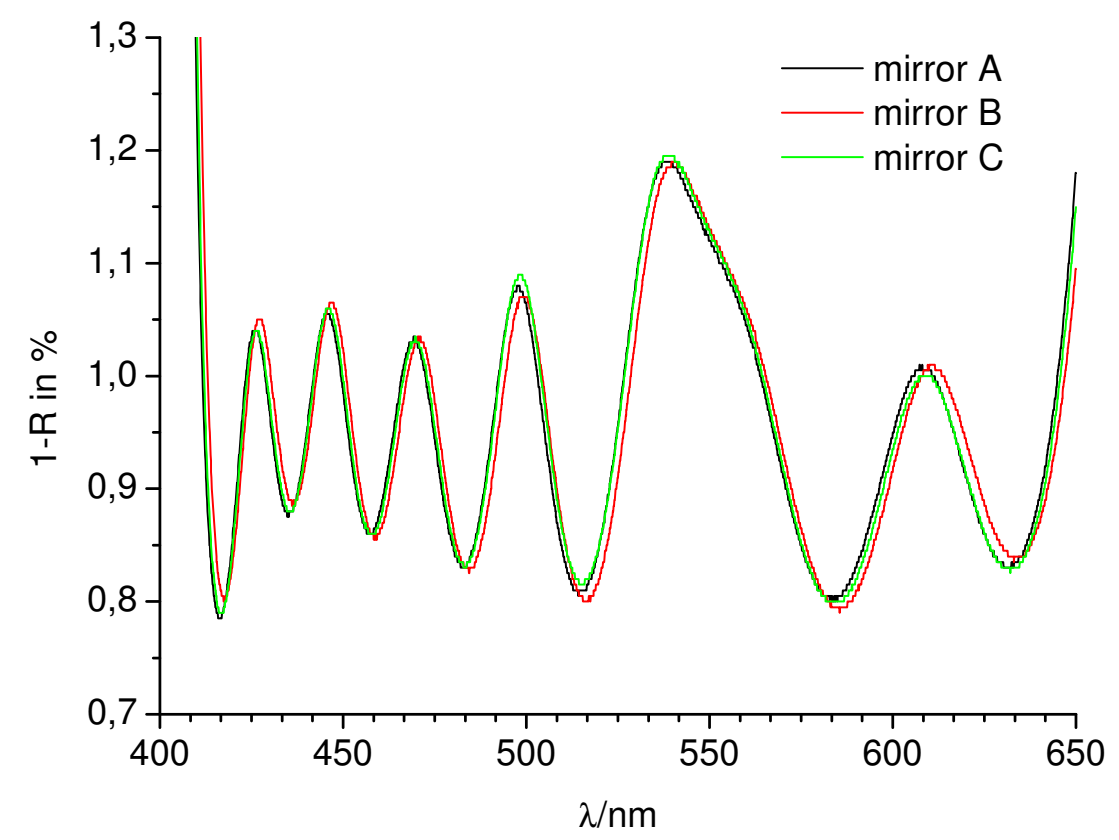

Figure A.1: Reflectivity of the Layertec mirrors $\left(R=99.0_{-0.2}^{+0.1} \%\right.$, wavelength region $\overline{415-640 \mathrm{~nm}}, \varnothing 25 \mathrm{~mm}$, radius of curvature $|r|=200 \mathrm{~mm}, \lambda / 4$ surface). 
had different spatial positions in this charge, which explains the slightly different reflections values for this spectra.

The mirrors shown in Fig. A.2 were produced by "Research Electro-Optics, Inc." (REO) in Boulder, Colorado, USA. They were used for the IBBCEAS measurements of azulene in a supersonic jet (section 6). Their reflectivity is so high $(R \geq 0.9999)$ that the detector of the spectrometer was not sensitive enough to measure the low light intensity transmitted by the mirrors. Therefore the data of Fig. A.2 was used only to assess the high reflectivity region of the mirrors.

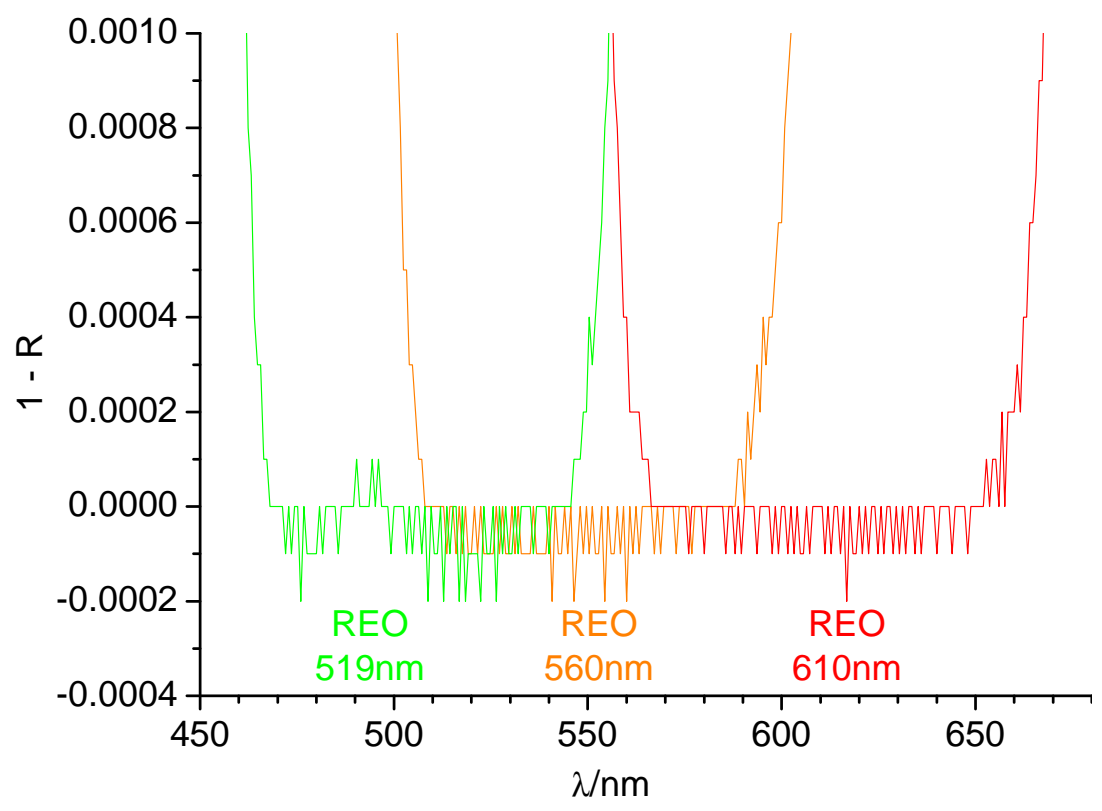

Figure A.2: Reflectivity of the REO mirrors $(R \approx 0.9999$, centre wavelength: $512 \mathrm{~nm}$ (green), $560 \mathrm{~nm}$ (orange) and $610 \mathrm{~nm}$ (red), $\varnothing 25 \mathrm{~mm}$, radius of curvature $|r|=1000 \mathrm{~mm}$, $1000 \mathrm{~mm}$ and $2000 \mathrm{~mm}$ respectively. 


\section{A.2 Nonlinear Chemical System}

The following reaction scheme was proposed in Gash04 as an explanation for the observed oscillating absorption in a naphthalene-gas-mixture after multi-UVphoton excitation. It consists of a pseudo-first order reaction followed by two consecutive unimolecular reactions.

$$
\begin{array}{rlr}
\mathrm{Q}+\mathrm{S} & \rightarrow \mathrm{A} & \text { rate }=k_{0} s q \\
\mathrm{~A} & \rightarrow \mathrm{B} & \text { rate }=k_{\mathrm{u}} a \\
\mathrm{~B} & \rightarrow \mathrm{C} & \text { rate }=k_{2} b
\end{array}
$$

$\mathrm{Q}$ and $\mathrm{S}$ are the reactants, $\mathrm{C}$ is the product, $\mathrm{A}$ and $\mathrm{B}$ are the intermediates. Initially, the system contains only $\mathrm{Q}$ and $\mathrm{S}$, at concentrations of $q_{0}$ and $s_{0}$ respectively. $\mathrm{S}$ is assumed to be present in stoichiometric excess, so its concentration does not change greatly as the reaction progresses. The concentrations of $\mathrm{A}, \mathrm{B}$ and $\mathrm{C}$ are zero initially. The system is closed and is isothermal. This scheme can be extended with the introduction of an autocatalytic step to display nonlinear dynamics.

The rate equation for the concentration of $\mathrm{Q}$ is given by

$$
\dot{q} \equiv \frac{\mathrm{d} q}{\mathrm{dt}}=-k_{0} s q
$$

Letting $k_{0} s_{0}=k_{\mathrm{e}}$, Eqn. A-4 can be written as:

$$
\begin{aligned}
\dot{q} & =-k_{0} s_{0} q \\
& =-k_{\mathrm{e}} q
\end{aligned}
$$


$k_{\mathrm{e}}$ is an effective rate coefficient. This first order differential equation can be solved to give $q$ as a function of time,

$$
q(t)=q_{0} e^{-k_{\mathrm{e}} t}
$$

$q$ decays exponentially from $q_{0}$, the rate of the decay is determined by $k_{\mathrm{e}}$. The rate equation for the concentration of the intermediate $\mathrm{A}$, $a$, is given by

$$
\dot{a}=k_{\mathrm{e}} q-k_{\mathrm{u}} a
$$

This equation can be solved to give an analytic equation for $a$.

$$
a(t)=\frac{k_{\mathrm{e}} q_{0}}{k_{\mathrm{u}}-k_{\mathrm{e}}}\left(e^{-k_{\mathrm{e}} t}-e^{-k_{\mathrm{u}} t}\right)
$$

The rate equation for the concentration of the intermediate $\mathrm{B}$ is given by

$$
\dot{b}=k_{\mathrm{u}} a-k_{2} b
$$

This equation can be solved Gash04 to give an analytic equation for $b$.

$$
b(t)=\frac{k_{\mathrm{e}} k_{\mathrm{u}} q_{0}}{k_{\mathrm{u}}-k_{\mathrm{e}}}\left[\left(\frac{e^{-k_{\mathrm{e}} t}-e^{-k_{2} t}}{k_{2}-k_{\mathrm{e}}}\right)-\left(\frac{e^{-k_{\mathrm{u}} t}-e^{-k_{2} t}}{k_{2}-k_{\mathrm{u}}}\right)\right]
$$

The absorption coefficient $\alpha$ is proportional to the cross section $\sigma$ for the absorption transition and the concentration, i.e. $\alpha_{a}=\sigma_{a} a$ and $\alpha_{b}=\sigma_{b} b$. And therefore

$$
\alpha_{a}(t)=\sigma_{a} \frac{k_{\mathrm{e}} q_{0}}{k_{\mathrm{u}}-k_{\mathrm{e}}}\left(e^{-k_{\mathrm{e}} t}-e^{-k_{\mathrm{u}} t}\right)
$$

and

$$
\alpha_{b}(t)=\sigma_{b} \frac{k_{\mathrm{e}} k_{\mathrm{u}} q_{0}}{k_{\mathrm{u}}-k_{\mathrm{e}}}\left[\left(\frac{e^{-k_{\mathrm{e}} t}-e^{-k_{2} t}}{k_{2}-k_{\mathrm{e}}}\right)-\left(\frac{e^{-k_{\mathrm{u}} t}-e^{-k_{2} t}}{k_{2}-k_{\mathrm{u}}}\right)\right]
$$


as well as the difference

$$
\begin{array}{cc}
\Delta \alpha_{a, b}(t)= & \sigma_{a} \frac{k_{\mathrm{e}} q_{0}}{k_{\mathrm{u}}-k_{\mathrm{e}}}\left(e^{-k_{\mathrm{e}} t}-e^{-k_{\mathrm{u}} t}\right) \\
& -\sigma_{b} \frac{k_{\mathrm{e}} k_{\mathrm{u}} q_{0}}{k_{\mathrm{u}}-k_{\mathrm{e}}}\left[\left(\frac{e^{-k_{\mathrm{e}} t}-e^{-k_{2} t}}{k_{2}-k_{\mathrm{e}}}\right)-\left(\frac{e^{-k_{\mathrm{u}} t}-e^{-k_{2} t}}{k_{2}-k_{\mathrm{u}}}\right)\right]
\end{array}
$$

can be used to fit the time response of the absorption using the rate constants of the chemical system as fit constants.

\section{A.3 Azulene: Vibronic Absorption Lines}

The vibronic absorption lines measured in a pulsed supersonic jet by CRDS from [Ruth99 are summarised in Table A.1.

The energy of the $S_{1, \nu}$ vibration modes above the $S_{1} \leftarrow S_{0}$ band origin at $14283.3 \mathrm{~cm}^{-1}$ is given in wavenumbers $\tilde{\nu}_{\text {vib. }}$. The relative absorption strengths (rel. abs. str.) of 35 modes used to simulate the azulene spectrum presented in section 6.6 are marked by an "y". The cases when the absorption strengths were reported to be very weak and close to the noise are marked by an " $\mathrm{y}^{*}$ " instead, and the relative absorption strengths were assumed to be 0.01 for the calculation (given in brackets):

Table A.1: Azulene in a Supersonic jet measured with CRDS:

$\mathrm{S}_{1}$ vibronic absorption lines and assignments from Ruth99.

\begin{tabular}{c|c|c|c|c} 
No. & $\tilde{\nu}_{\text {vib }} / \mathrm{cm}^{-1}$ & rel. abs. str. & used & assignment \\
\hline 1 & 0.0 & 1.000 & - & $0_{0}^{0}$ origin \\
2 & 228.0 & 0.044 & $\mathrm{y}$ & $39_{1}^{0}$ \\
3 & 330.4 & $(0.010)$ & $\mathrm{y}^{*}$ & $38_{0}^{1}$ \\
4 & 363.6 & $(0.010)$ & $\mathrm{y}^{*}$ & n.a.
\end{tabular}




\begin{tabular}{|c|c|c|c|c|}
\hline No. & $\tilde{\nu}_{\mathrm{vib}} / \mathrm{cm}^{-1}$ & rel. abs. str. & used & assignment \\
\hline 5 & 376.6 & 0.075 & $\mathrm{y}$ & $17_{0}^{1}$ \\
\hline 6 & 381.9 & 0.061 & $\mathrm{y}$ & $23_{0}^{2}$ \\
\hline 7 & 495.7 & $(0.010)$ & $\mathrm{y}^{*}$ & $37_{0}^{1}$ \\
\hline 8 & 606.7 & $(0.010)$ & $y^{*}$ & n.a. \\
\hline 9 & 615.3 & $(0.010)$ & $\mathrm{y}^{*}$ & n.a. \\
\hline 10 & 661.3 & 0.405 & $\mathrm{y}$ & $16_{0}^{1}$ \\
\hline 11 & 771.7 & $(0.010)$ & $\mathrm{y}^{*}$ & $36_{0}^{1}$ \\
\hline 12 & 858.7 & 0.475 & $\mathrm{y}$ & $15_{0}^{1}$ \\
\hline 13 & 910.8 & 0.043 & $\mathrm{y}$ & $14_{0}^{1}$ \\
\hline 14 & 949.0 & $(0.010)$ & $\mathrm{y}^{*}$ & n.a. \\
\hline 15 & 1039.3 & 0.065 & $\mathrm{y}$ & $35_{0}^{1}\left(17_{0}^{1} 16_{0}^{1}\right)$ \\
\hline 16 & 1068.0 & 0.075 & $\mathrm{y}$ & $34_{0}^{1}$ \\
\hline 17 & 1194.8 & 0.219 & $\mathrm{y}$ & $13_{0}^{1}$ \\
\hline 18 & 1223.9 & 0.098 & $\mathrm{y}$ & $12_{0}^{1}$ \\
\hline 19 & 1237.4 & 0.080 & $\mathrm{y}$ & $33_{0}^{1}\left(17_{0}^{1} 15_{0}^{1}\right)$ \\
\hline 20 & 1322.6 & 0.076 & - & $16_{0}^{2}$ \\
\hline 21 & 1344.2 & 0.059 & $\mathrm{y}$ & $32_{0}^{1}$ \\
\hline 22 & 1394.1 & 0.294 & $\mathrm{y}$ & $10_{0}^{1}$ \\
\hline 23 & 1405.6 & 0.204 & $\mathrm{y}$ & $31_{0}^{1}$ \\
\hline 24 & 1449.5 & 0.077 & $\mathrm{y}$ & $30_{0}^{1}$ \\
\hline 25 & 1520.0 & 0.237 & - & $16_{0}^{1} 15_{0}^{1}$ \\
\hline 26 & 1545.0 & 0.160 & $\mathrm{y}$ & $29_{0}^{1}$ \\
\hline 27 & 1559.2 & 0.457 & $\mathrm{y}$ & $9_{0}^{1}$ \\
\hline 28 & 1569.4 & 0.250 & $\mathrm{y}$ & $8_{0}^{1}\left(\begin{array}{ll}16_{0}^{1} & \left.14_{0}^{1}\right)\end{array}\right.$ \\
\hline 29 & 1717.5 & 0.199 & - & $15_{0}^{2}$ \\
\hline 30 & 1772.1 & 0.124 & - & $15_{0}^{1} 14_{0}^{1}\left(17_{0}^{1} 10_{0}^{1}\right)$ \\
\hline
\end{tabular}




\begin{tabular}{|c|c|c|c|c|}
\hline No. & $\tilde{\nu}_{\mathrm{vib}} / \mathrm{cm}^{-1}$ & rel. abs. str. & used & assignment \\
\hline 31 & 1800.6 & 0.230 & $\mathrm{y}$ & $7_{0}^{1}$ \\
\hline 32 & 1853.2 & 0.139 & - & $16_{0}^{1} 13_{0}^{1}$ \\
\hline 33 & 1883.2 & 0.126 & - & $16_{0}^{1} 12_{0}^{1}$ \\
\hline 34 & 1925.8 & 0.092 & - & $15_{0}^{1} 34_{0}^{1}$ \\
\hline 35 & 1941.2 & 0.094 & $\mathrm{y}$ & f. 1 \\
\hline 36 & 2052.7 & 0.400 & - & $15_{0}^{1} 13_{0}^{1}$ \\
\hline 37 & 2065.2 & 0.186 & - & $16_{0}^{1} 31_{0}^{1}$ \\
\hline 38 & 2082.1 & 0.165 & $\mathrm{y}$ & f. $2\left(\begin{array}{ll}15_{0}^{1} & \left.12_{0}^{1}\right)\end{array}\right.$ \\
\hline 39 & 2112.7 & 0.115 & - & $16_{0}^{1} 30_{0}^{1}$ \\
\hline 40 & 2176.8 & 0.250 & - & $17_{0}^{1} 7_{0}^{1}$ \\
\hline 41 & 2216.0 & 0.366 & $\mathrm{y}$ & f.3 $\left(16_{0}^{1} 9_{0}^{1}\right)$ \\
\hline 42 & 2249.6 & 0.415 & - & $15_{0}^{1} 10_{0}^{1}$ \\
\hline 43 & 2264.0 & 0.226 & - & $15_{0}^{1} 31_{0}^{1}$ \\
\hline 44 & 2308.4 & 0.147 & - & n.a. \\
\hline 45 & 2382.6 & 0.201 & - & n.a. \\
\hline 46 & 2415.3 & 0.436 & $\mathrm{y}$ & f. $4\left(15_{0}^{1} 9_{0}^{1}\right)$ \\
\hline 47 & 2458.6 & 0.191 & - & $16_{0}^{1} 7_{0}^{1}$ \\
\hline 48 & 2515.7 & 0.101 & - & $16_{0}^{2} 13_{0}^{1}$ \\
\hline 49 & 2585.9 & 0.214 & - & $16_{0}^{1} 15_{0}^{1} 34_{0}^{1}$ \\
\hline 50 & 2617.9 & 0.170 & - & $12_{0}^{1} 10_{0}^{1}$ \\
\hline 51 & 2658.7 & 0.183 & - & $15_{0}^{1} 7_{0}^{1}$ \\
\hline 52 & 2712.5 & 0.193 & $\mathrm{y}$ & f.5 \\
\hline 53 & 2752.5 & 0.227 & - & f.6 $\left(13_{0}^{1} 9_{0}^{1}\right)$ \\
\hline 54 & 2781.6 & 0.268 & - & f. $7\left(33_{0}^{1} 29_{0}^{1}\right)$ \\
\hline 55 & 2840.1 & 0.132 & - & $35_{0}^{1} 7_{0}^{1}$ \\
\hline 56 & 2876.8 & 0.170 & $\mathrm{y}$ & f. 8 \\
\hline
\end{tabular}




\begin{tabular}{c|c|c|c|c} 
No. & $\tilde{\nu}_{\mathrm{vib}} / \mathrm{cm}^{-1}$ & rel. abs. str. & used & assignment \\
\hline 57 & 2911.5 & 0.255 & $\mathrm{y}$ & $\mathrm{f} .9$ \\
58 & 2935.4 & 0.266 & $\mathrm{y}$ & $(\mathrm{f} .10)\left(17_{0}^{1} 16_{0}^{1} 15_{0}^{1} 35_{0}^{1}\right)$ \\
59 & 2946.4 & 0.289 & $\mathrm{y}$ & $(\mathrm{f} .11)$
\end{tabular}




\section{Bibliography}

[Allain96] T. Allain, S. Leach and E. Sedlmayr. "Photodestruction of PAHs in the interstallar medium I". Astronomy and Astrophysics 305 (1996) 602-615.

[Anderson74] J.B. Anderson. Molecular beams from nozzle sources in: Molecular beams and low density gas dynamics, Vol. 4, Ed. P.P. Wegener, Marcel Dekker. Inc., New York (1974).

[Ball03] S.M. Ball and R.L. Jones. "Broad-Band Cavity Ring-Down Spectroscopy". Chem. Rev. 103 (2003) 5239.

[Ball04] S.M. Ball and R.L. Jones. "Broadband cavity enhanced absorption spectroscopy using light emitting diodes". Chem. Phys. Lett. 398 (2004) 68.

[Bearpark96] M.J. Bearpark, F. Bernardi, S. Clifford, M. Olivucci, M.A. Robb, B.R. Smith and T. Vreven. "The Azulene $\mathrm{S}_{1}$ State Decays via a Conical Intersection: A CASSCF Study with MMVB Dynamics". J. Am. Chem. Soc. 118 (1996) 169.

[Becker-Ross97] H. Becker-Ross and S. V. Florek. "Echelle spectrometers and charge-coupled devices". Spectrochim. Acta Part B 52 (1997) 1367.

[Berden00] G. Berden, R. Peeters and G. Meijer. "Cavity ring-down spectroscopy: Experimental schemes and applications". Int. Rev. Phys. Chem. 19 (2000) 565. 
[Biennier03] L. Biennier, F. Salama, L.J. Allamandola and J.J. Scherer. "Pulsed discharge nozzle cavity ringdown spectroscopy of cold polycyclic aromatic hydrocarbon ions". J. Chem. Phys. 118 (2003) 7863 .

[Bjorklund80] G.C. Bjorklund. "Frequency modulation spectroscopy: a new method for measuring weak absorptions and dispersions". Opt. Lett. 5 (1980) 15.

[Bockhorn94] H. Bockhorn. Soot formation in combustion. Springer Verlag, Berlin (1994).

[Bohren83] C.F. Bohren and D.R. Huffman. Absorption and Scattering of Light by Small Particles. Wiley-Interscience, New York (1983).

[Born80] M. Born and E. Wolf. Principles of Optics, 6th Ed. Pergamon Press, New York (1980).

[Busch99] K.W. Busch and M.A. (Eds.) Busch. Cavity-ringdown spectroscopy. An ultra-trace-absorption measurement technique, American Chemical Society, Symposium Series 720,. Oxford University Press, Oxford (1999).

[Chernin01] S.M. Chernin. "Promising version of the three-objective multipass matrix system". J. Mod. Opt. 48 (2001) 619.

[Cheskis99] S. Cheskis. "Quantitative measurements of absolute concentrations of intermediate species in flames". Progress in Energy and Combustion Science 25 (1999) 233-252.

[Crosson99] E.R. Crosson, P. Haar, G.A. Marcus, H.A. Schwettman, B.A. Paldus, T.G. Spence and R.N. Zare. "Pulse-stacked cavity ringdown spectroscopy". Rev. Sci. Instr. 70 (1999) 4.

[Cui00] W. Cui, B. Hadas, B. Cao and C. Lifshitz. "Time-Resolved Photodissociation (TRPD) of the Naphthalene and Azulene Cations in an Ion Trap/Reflectron". J. Phys. Chem. A 104 (2000) 6339. 
[Czyzewski01] Czyzewski, S. A. Chudzynski, K. Ernst, G. Karasinski, L. Kilianek, A. Pietruczuk, W. Skubiszak, T. Stacewicz, K. Stelmaszczyk, B. Koch and P. Rairoux. "Cavity ring-down spectrography". Opt. Comm. 191 (2001) 271-275.

[Demtröder88] W. Demtröder. Laser Spectroscopy, Basic Concepts and Instrumentation. Springer-Verlag, Berlin, Heidelberg, New York (1988).

[Demtröder03] W. Demtröder. Molekülphysik. Oldenbourg Wissenschaftverlag, München, Wien (2003).

[Engeln96] R. Engeln and G. Meijer. "A Fourier transform Cavity Ring Down spectrometer". Rev. Sci. Instr. 67 (1996) 2708.

[Engeln97a] R. Engeln, G. Berden, E. van den Berg and G. Meijer. "Polarization Dependent Cavity Ring Down Spectroscopy." J. Chem. Phys. 107 (1997) 4458.

[Engeln97b] R. Engeln, E. van den Berg, G. Meijer, L. Lin, G.M.H. Knippels and A.F.G. van der Meer. "Cavity Ring Down Spectroscopy with a Free-Electron Laser". Chem. Phys. Lett. 269 (1997) 293.

[Engeln98] R. Engeln, G. Berden, R. Peeters and G. Meijer. "Cavity enhanced absorption and cavity enhanced magnetic rotation spectroscopy". Rev. Sci. Instr. 69 (1998) 3763-3769.

[Engeln99] R. Engeln, G. von Helden, A.J.A. van Roij and G. Meijer. "Cavity ring down spectrocopy on solid $\mathrm{C}_{60}$ ". J. Chem. Phys. 110 (1999) 2732 .

[Epstein98] I.R. Epstein and J.A. Pojman. An Introduction to Nonlinear Chemical Dynamics: Oscillations Waves, Patterns and Chaos. Oxford University Press, New York (1998).

[Field85] R.J. Field and M. Burger. Oscillations and Traveling Waves in Chemical Systems. John Wiley and Sons, New York (1985). 
[Fluendy73] M.A.D. Fluendy and K.P. Lawley. Chemical applications of molecular beam scattering. Chapman and Hall, London (1973).

[G. Rempe92] H.J. Kimble G. Rempe, R.J. Thompson and R. Lalezari. "Measurement of Ultralow Losses in an Optical Interferometer". Opt. Lett. 17 (1992) 363-365.

[Gash04] E.W. Gash. Unusual dynamic absorption of naphthalene-buffer gas mixtures following UV photolysis. PhD. Thesis, University College Cork, Cork, Ireland (2004).

[Haarhoff63] P.C. Haarhoff. "The density of vibrational energy levels of polyatomic molecules". Mol. Phys. 7 (1963) 101.

[Hall81] J.L. Hall, L. Hollberg, T. Baer and H.G. Robinson. "Optical heterodyne saturation spectroscopy". Appl. Phys. Lett. 39 (1981) 680.

[Hallock02] A. J. Hallock, E. S. F. Berman and R. N. Zare. "Direct Monitoring of Absorption in Solution by Cavity Ring-Down Spectroscopy". Anal. Chem. 74 (2002) 1741-1743.

[Hallock03] A. J. Hallock, E. S. F. Berman and R. N. Zare. "Use of Broadband, Continious-Wave Diode Lasers in Cavity Ring-Down Spectroscopy for Liquid Samples". Appl. Spectr. 57 (2003) 571-573.

[Hamers02] E. Hamers, D. Schraam and R. Engeln. "Fourier Transform Phase Shift Cavity Ring Down Spectroscopy". Chem. Phys. Lett. 365 (2002) 237-243.

[Herriot65] D.R. Herriot and H.J. Schulte. "Folded Optical Delay Lines". Appl. Opt. 4 (1965) 883-889.

[Hitran98] Hitran, L.S. Rothman, C.P. Rinsland, A. Goldman, S.T. Massie, D.P. Edwards, J.-M. Flaud, A. Perrin, C. Camy-Peyret, V. Dana, J.-Y. Mandin, J. Schroeder, A. McCann, R.R. Gamache, R.B. 
Wattson, K. Yoshino, K.V. Chance, K.W. Jucks, L.R. Brown, V. Nemtschinov and P. Varanasi. "http://www.hitran.com". J. Quant. Spectrosc. Rad. Transfer 60 (1998) 665.

[Hodges96] J.T. Hodges, J.P. Looney and R.D. van Zee. "Response of a ringdown cavity to an arbitrary excitation". J. Chem. Phys. 105 (1996) 10278.

[Hoheisel03] G. Hoheisel, E. Heinecke and A. Hese. "Optical-optical double resonance spectroscopy on pentacene molecules in a supersonic jet". Chem. Phys. Lett. 373 (2003) 416-421.

[Hollas95] J.M. Hollas, D. Phillips and (Editors). Jet spectroscopy and molecular dynamics. Chapman \& Hall, London (1995).

[Hollas96] J.M. Hollas. Modern Spectroscopy. John Wiley and Sons Ltd, ISBN 0471965235 (1996).

[Hunt62] G.R. Hunt and Ross I.G. "Spectrum of Azulene, Part II. The 7000-A and 3500-A Absorption Systems". J. Mol. Spectrosc. 9 (1962) 50 .

[Jochims92] H.W. Jochims, H. Rasekh, E. Ruehl and H. Baumgaertel. "The photofragmentation of naphthalene and azulene monocations in the energy range 7-22 eV". Chem. Phys. 168 (1992) 159.

[Kleine99] D. Kleine, S. Stry, J. Lauterbach, K. Kleinermanns and P. Hering. "Measurement of the absolute intensity of the fifth $\mathrm{CH}$ stretching overtone of benzene using cavity ring-down spectroscopy". Chem. Phys. Lett. 312 (1999) 185-190.

[Lambert77] J.D. Lambert. Vibrational and rotational relaxation in gases. Claredon Press, Oxford (1977).

[Lehmann96] K.K. Lehmann and D. Romanini. "The superposition principle and cavity ring-down spectroscopy". J. Chem. Phys. 105 (1996) 10263. 
[Ma99] L.S. Ma, J. Ye, P. Dube and J.L. Hall. "Ultrasensitive frequencymodulation spectroscopy enhanced by a high-finesse optical cavity: theory and application to overtone transitions of $\mathrm{C}_{2} \mathrm{H}_{2}$ and $\mathrm{C}_{2}$ HD,". J. Opt. Soc. Am. B 16 (1999) 2255-2268.

[Mat] Software package MATHEMATICA v4.1, 2000. Wolfram Research (Inc.), Champaign, IL, USA, www.wolfram.com.

[Meijer94] G. Meijer, M.G.H. Boogaarts, R.T. Jongma, D.H. Parker and A.M. Wodtke. "Coherent Cavity Ring Down Spectroscopy". Chem. Phys. Lett. 217 (1994) 112.

[Miller80] J.H. Miller, L. Andrews, P.A. Lund and P.N. Schatz. "Argon matrix photolysis and photoionization studies of benzene. Absorption spectrum of benzene cation and benzene dimer cation". J. Chem. Phys. 73 (1980) 4932.

[Miller88] D.R. Miller. Free jet sources in: Atomic and molecular beam methods, Vol. 1, Eds. G. Scoles, D. Bassi, U. Buck, D. Lainé. Oxford University Press, Oxford (1988).

[Miller97] G.P. Miller and C.B. Winstead. "Inductively Coupled Plasma Cavity Ringdown Spectroscopy". J. Anal. Atom. Spect. 12 (1997) 907.

[Motylewski99] T. Motylewski and H. Linnartz. "Cavity ring down spectroscopy on radicals in a supersonic slit nozzle discharge". Rev. Sci. Instr. 70 (1999) 1305.

[Muir03] R.N. Muir and A.J. Alexander. "Structure of monolayer dye films studied by Brewster angle cavity ringdown spectroscopy". Phys. Chem. Chem. Phys. 5 (2003) 1279.

[Naus00] H. Naus and W. Ubachs. "Experimental Verification of RayleighScattering Cross sections". Opt. Lett. 25 (2000) 347-349. 
[Negri93] F. Negri and M.Z. Zgierski. "On the vibronic structure of the $\mathrm{S}_{0} \leftrightarrow \mathrm{S}_{1}$ transitions in azulene". J. Chem. Phys. 99 (1993) 4318.

[Newnham98] D.A. Newnham and J. Ballard. "Visible absorption cross section and integrated absorption intensities of molecular oxygen $\left(\mathrm{O}_{2}\right.$ and $\left.\mathrm{O}_{4}\right)$ ). J. Geophys. Res. 103 (1998) 28801-28816.

[O'Keefe88] A. O'Keefe and D.A.G. Deacon. "Cavity Ring-Down optical spectrometer for absorption measurements using pulsed laser sources". Rev. Sci. Instr. 59 (1988) 2544.

[O'Keefe98] A. O'Keefe. "Integrated cavity output analysis of ultra-weak absorption". Chem. Phys. Lett. 293 (1998) 331-336.

[O'Keefe99] A. O'Keefe, J.J. Scherer and J.B. Paul. "CW integrated cavity output spectroscopy". Chem. Phys. Lett. 307 (1999) 343-349.

[Olivucci99] M. Olivucci. private communication to A. Ruth (1999).

[Patel79] C.K.N. Patel and A.C. Tam. "Optoacoustic spectroscopy of liquids". Appl. Phys. Lett. 34 (1979) 467.

[Paul99] J.B. Paul, R.A. Provencal, C. Chapo, K. Roth, R. Casaes and R.J. Saykally. "Infrared Cavity Ringdown Spectroscopy of the Water Cluster Bending Vibrations". J. Phys. Chem. A 103 (1999) 2972.

[Pino99] T. Pino, N. Boudin and P. Bréchignac. "Electronic spectrum of cold Naphthalene cation by photodissociation of its Van der Waals complexes in a molecular beam". J. Chem. Phys. 111 (1999) 7337.

[Pipino00] A.C.R. Pipino. "Monolithic Folded Resonator for Evanescent Wave Cavity Ringdown Spectroscopy". Appl. Opt. 39 (2000) 1449. 
[Platt94] U. Platt. "Differential Optical Absorption Spectroscopy (DOAS)". Air Monitoring by Spectroscopic Techniques, Chemical Analysis Series 127 (1994) 27.

[Reddy82] K.V. Reddy, D.F. Heller and M.J. Berry. "Highly vibrationally excited benzene: Overtone spectroscopy and intramolecular dynamics of $\mathrm{C}_{6} \mathrm{H}_{6}, \mathrm{C}_{6} \mathrm{D}_{6}$ and partially deuterated or substituted benzenes". J. Chem. Phys. 76 (1982) 2814-2837.

[Reid78] J. Reid, B.K. Garside, J. Shewchun, M. El-Sherbiny and E.A. Ballik. "High sensitivity point monitoring of atmospheric gases employing tunable diode lasers". Appl. Opt. 17 (1978) 1806.

[Romanini95] D. Romanini. "Very high sensitive absorption spectroscopy: ring down cavity and intracavity laser absorption". Ann. Phys. (France) 20 (1995) 665-674.

[Romanini97a] D. Romanini, A.A. Kachanov, A.A. Sadeghi and F. Stoeckel. "CW Cavity Ring Down Spectroscopy". Chem. Phys. Lett. 264 (1997) 316-322.

[Romanini97b] D. Romanini, A.A. Kachanov and F. Stoeckel. "Cavity ringdown spectroscopy: broad band absolute absorption measurements". Chem. Phys. Lett. 270 (1997) 546-550.

[Romanini99] D. Romanini, L. Bienner, F. Salama, A. Kachanov, L.J. Allamandola and F. Stoeckel. "Jet-Discharge Cavity Ring-down Spectroscopy of Ionized Polycyclic Aromatic Hydrocarbons: Progress in Testing the PAH Hypothesis for the Diffuse Interstellar Band Problem". Chem. Phys. Lett. 303 (1999) 165.

[Ruth99] A.A. Ruth, E.-K. Kim and A. Hese. "The $\mathrm{S}_{0} \rightarrow \mathrm{S}_{1}$ cavity ringdown absorption spectrum of jet-cooled azulene: dependence of internal conversion on the excess energy". Phys. Chem. Chem. Phys. 22 (1999) 5121. 
[Ruth00] A.A. Ruth. "Laser spectroscopy of jet-cooled aromatic hydrocarbons and thiocabonyl compounds". Habilitationsschrift, TUBerlin, (2000).

[Ruth02a] A.A. Ruth. "private communication" (2002).

[Ruth02b] A.A. Ruth, E.W. Gash, M. Staak and S.E. Fiedler. "Multi-photon UV photolysis of naphthalene-gas mixtures: A new gas-phase oscillatory system". Phys. Chem. Chem. Phys. 4 (2002) 52175220 .

[Salama91] F. Salama and L. J. Allamandola. "Electronic absorption spectroscopy of matrix-isolated polycyclic aromatic hydrocarbon cations. I. The naphthalene cation $\left(\mathrm{C}_{10} \mathrm{H}_{8}^{+}\right)$". J. Chem. Phys. 94 (1991) 6964-6977.

[Sappey98] A.D. Sappey, E.S. Hill, T. Settersten and M.A. Linne. "Fixedfrequency cavity ringdown diagnostic for atmospheric particulate matter". Optics Lett. 23 (1998) 954.

[Scherer95] J.J. Scherer, J.B. Paul, C.P. Collier and R.J. Saykally. "Cavity Ringdown Laser Absorption Spectroscopy (CRLAS) and Timeof-Flight Mass Spectroscopy of Jet-cooled Silver Silicides,". J. Chem. Phys. 103 (1995) 113.

[Scherer97] J.J. Scherer, J.B. Paul, A. O'Keefe and Saykally R.J. "Cavity Ringdown Laser Absorption Spectroscopy: History, Development and Application to Pulsed Molecular Beams". Chem. Rev. 97 (1997) 25-51.

[Scherer98] J.J. Scherer. "Ringdown spectral photography". Chem. Phys. Lett. 292 (1998) 143-153.

[Schott04] Schott. "Optical Glass Catalogue, Data Table. The stated value was determined as the average of several melts." Mainz, Germany, www.schott.de (2004). 
[Scott90] S.K. Scott and P.M. Gray. Chemical Oscillations and Instabilities. Oxford University Press, Oxford (1990).

[Scott94] S.K. Scott. Oscillations, Waves and Chaos in Chemical Kinetics. Oxford University Press, Oxford (1994).

[Sircar96] A. Sircar, R. K. Dwivedi and R. K. Thareja. "A numerical investigation of the dependence of the threshold irradiance on the wavelength in laser-induced breakdown in $\mathrm{N}_{2}$ ". Appl. Phys. B 63 (1996) 623 .

[Snyder03] K. L. Snyder and R. N. Zare. "Cavity Ring-Down Spectroscopy as a Detector for Liquid Chromatography". Anal. Chem. 75 (2003) 3086-3091.

[Stein72] S.E. Stein and B.S. Rabinovitch. "Accurate evaluation of internal energy sums and densities including anharmonic oscillators and hindered rotors". J. Chem. Phys. 58 (1972) 2438.

[Steyert01] D.W. Steyert, J.M. Sirota, M.E. Mickelson and D.C. Reuter. "Two new long-pass cells for infrared and visible spectroscopy". Rev. Sci. Instr. 72 (2001) 4337-4347.

[Stone78] J. Stone. "Absorption curve of the $607 \mathrm{~nm}$ line in benzene". Appl. Opt. 17 (1978) 2876-2877.

[Stover95] J.C. Stover. Optical scattering: measurement and analysis. SPIE Optical Engineering Press, Bellingham, Wash., USA (1995).

[Suzuki96] T. Suzuki, T. Ichimura, M. Kusaba and N. Nakashima. "Reaction dynamics of hot naphthalene molecule in the gas phase with ArF laser excitation". Chem. Phys. Lett. 263 (1996) 197.

[Swofford76] R.L. Swofford, M.E. Long and A.C. Albrecht. "C-H vibrational states of benzene, naphthalene, and anthracene in the visible region by thermal lensing spectroscopy and the local mode model". J. Chem. Phys. 65 (1976) 179. 
[Tong03] Zhaoguo Tong, M. Jakubinek, A. Wright, A. Gillies and H.-P. Loock. "Fiber-loop ring-down spectroscopy: a sensitive absorption technique for small liquid samples". Rev. Sci. Instr. $\mathbf{7 4}$ (2003) 4818-26.

[Turcu97] I.C.E. Turcu, M.C. Gower and P. Huntington. "Measurement of $\mathrm{KrF}$ laser breakdown threshold in gases". Optics Comm. 135 (1997) 66.

[vanderLinde98] P.R. van der Linde, J.G. Blok and H.A.J. Oonk. "Naphthalene as a reference substance for vapour pressure measurements looked upon from an unconventional point of view". J. Chem. Thermodynamics 30 (1998) 909.

[Werle98] P. Werle. "A review of recent advances in semiconductor laser based gas monitors". Spectrochim. Acta Part A 54 (1998) 197236.

[Wheeler98] M.D. Wheeler, S.M. Newman, A.J. Orr-Ewing and M.N.R. Ashfold. "Cavity Ring-Down Spectroscopy". J. Chem. Soc. Faraday Trans. 94 (1998) 337.

[White42] J.U. White. "Long Optical Paths of Large Aperture". J. Opt. Soc. Am. 32 (1942) 285-288.

[White76] J.U. White. "Very Long Optical Paths in Air". J. Opt. Soc. Am. 66 (1976) 411-416.

[Wurzer99] A.J. Wurzer, T. Wilhelm, J. Piel and E. Riedle. "Comprehensive measurement of the $\mathrm{S}_{1}$ azulene relaxation dynamics and observation of vibrational wavepacket motion". Chem. Phys. Lett. 299 (1999) 296-302.

[Xu02] Shucheng Xu, Guohe Sha and Jinchun Xie. "Cavity ring-down spectroscopy in the liquid phase". Rev. Sci. Instr. 73 (2002) $255-258$. 
[Ye98] J. Ye, L.S. Ma and J.L. Hall. "Ultrasensitive detections in atomic and molecular physics: demonstration in molecular overtone spectroscopy". J. Opt. Soc. Am. B 15 (1998) 6.

[Ye00] J. Ye and J.L. Hall. "Cavity ringdown heterodyne spectroscopy: High sensitivity with microwatt light power". Phys. Rev. A $6 \mathbf{6 1}$ (2000) 061802.

[Zalicki95] P. Zalicki and R.N. Zare. "Cavity ring-down spectroscopy for quantitative absorption measurements". J. Chem. Phys. 102 (1995) 2708. 


\section{Curriculum Vitae}

1972

Sven Eberhard Fiedler is born on Juni $27^{\text {th }}$

Parents: Jörg Fiedler and Anke Dittberner

1978 - 1984 Elementary school in Berlin, Germany

1984 - 1991 Secondary school in Berlin, Germany, degree: Abitur

1991 - 1997 Studies in Physics at the Technical University of Berlin, Germany, degree: Physics Diploma

1994 - 1998 Student assistant for lab classes

1998 - 1999 Alternative civilian service at a sheltered workshop (Berliner Werkstätten für Behinderte)

1999

Research assistant at the Max-Born-Institute of Berlin Research field: Transient x-ray lasers

1999 - 2004 Research and teaching assistant of Prof. A. Hese at the Technical University of Berlin, Germany

Research field: Cavity-enhanced absorption spectroscopy of cold molecules in a supersonic jet

Teaching field: Physics for engineers, exercise courses and seminars for atomic and molecular physics, advanced lab classes

2002 - 2004 Frequent research stays at the University College Cork, Cork, Ireland 


\section{Acknowledgements}

- I would like to express my gratitude to Prof. Achim Hese (TU-Berlin), for his confidence in me, for continuous support and encouragement, for being open and many helpful discussions regarding the new concept of IBBCEAS, for the opportunity to develop the technique and to apply it to fundamental scientific research.

- Many thanks to Dr. Andy Ruth (University College Cork, Cork, Ireland) for the idea of IBBCEAS, the helpful discussions, the loan of mirrors and for tapping me on the shoulder.

- Thanks to Dr. Gerald Hoheisel (TU Berlin) for good teamwork, his good humored encouragement and of course for listening to my ideas and long discussions.

- I am grateful for the loan of a short-arc xenon lamp provided by Dr. Uwe Heitmann (ISAS-Berlin). Thanks as well to Prof. Hans Joachim Eichler for the possibility to use his spectrometer, and to Chriss Scharfenorth, who helped me a lot with mirror theory (TU-Berlin).

- Thanks to Rüdiger Schmidt (TU-Berlin) for the software and hardware support and to the members of the "Doppelkopf" team of the institute who were helpful in many ways: Dr. Elke Heinecke, Dr. Sophie Kröger, Dr. Ralf Müller, Tobias Richter, Marcel Dieckow and last but not least Robert Kastl (all TU-Berlin).

- Thanks to my father, Jörg Fiedler, who filled me with enthusiasm for science and supported me on the way, to my mother, Anke Dittberner, who let me go my own way and put fresh heart into me after unsuccessful experiments, and to my stepmother, Elvira Fiedler, who read the proofs. 